\title{
A SEARCH FOR A NEW CHARGED HEAVY VECTOR BOSON DECAYING TO A $\mu \nu$ PAIR
}

by

\author{
Hyunsoo Kim
}

\author{
A thesis submitted in conformity with the requirements \\ for the degree of Doctor of Philosophy, \\ Graduate Department of Physics, \\ University of Toronto
}

(c) Copyright by Hyunsoo Kim (1999) 


\begin{abstract}
A search has been performed for a new charged heavy vector boson produced in $p \bar{p}$ collisions at $\sqrt{s}=1.8 \mathrm{TeV}$ and upon observing no significant excess, limits on the cross section times branching fractions, $\sigma\left(p \bar{p} \rightarrow W^{\prime}\right) \cdot \mathcal{B}\left(W^{\prime} \rightarrow \mu \nu\right)$, are set at 95\% confidence level. Assuming that this new boson has standard model strength couplings, this search excludes a $W^{\prime}$ boson with mass less than $660 \mathrm{GeV} / \mathrm{c}^{2}$ at $95 \%$ confidence level.
\end{abstract}




\section{Acknowledgments}

I owe my deep gratitudes to my supervisor Prof. Pekka Sinervo for his encouragement, guidance, and patience. I benefited greatly from his enthusiasm and expertise in physics.

Of course, this work would not have been possible without the hard work of the CDF collaboration and supporting staffs at Fermilab. Especially, my thanks go to John Conway, Henry Frisch, Kaori Maeshima, David Stuart, and others at the exotic physics group at CDF for their encouragement and constructive criticism.

I thank Robert Cropp, Szymon Gadomski, Björn “9” Hinrichson, Andreas Hölscher, Kyung Kwang Joo, Andrew Robinson, Pierre Savard, and Wendy Taylor for making my stay at graduate school an interesting experience, Kostas Kordas for making my trips to Fermilab less painful, and Kurt Biery for his help in my service work for the collaboration. I would like to thank Prof. William Trischuk for his good humour and advice.

Many thanks go to George "Joe Pepsi" Sganos and Andreas Warburton for not only being good colleagues but also being great fellas. Without their friendship, keeping my sanity would have been an insurmountable task.

I thank my old friends Song Hun "Tocci" Choi and Jung Hee "Atom" Cho for being there to remind me how blissful it is being a clueless graduate student than a responsible and productive member of the society.

I acknowledge the financial support from the Natural Sciences and Engineering Research Council of Canada, the University of Toronto, and the Loyal Canadian Taxpayers.

Last but not least, I thank my family, Dad, Mom, Hyekyung, and Jungsoo, who have supported my endeavour unconditionally, even though I could not explain well what I was doing.

Hyunsoo 


\section{Contents}

Abstract $\quad$ iii

Acknowledgments $\quad$ iv

1 Introduction 1

1.1 A Brief History of the Standard Model . . . . . . . . . . . 1

1.2 Left-Right Symmetric Model . . . . . . . . . . . . . . 3

1.2.1 Symmetry Breaking in the Standard Model . . . . . . . . 3

1.2.2 Symmetry Breaking in Left-Right Symmetric Model . . . . . 5

1.3 Production and Decay of a $W^{\prime}$ Boson . . . . . . . . . . . 6

1.4 Previous Searches ........................ 10

1.5 Outline of Dissertation . . . . . . . . . . . . 12

2 The Experimental Apparatus $\quad 13$

2.1 The Tevatron Accelerator ................. 13

2.2 Overview of the CDF Detector . . . . . . . . . . 16

2.3 The Tracking Detectors . . . . . . . . . . . 18

2.4 The Calorimeters . . . . . . . . . . . . . 20

2.5 The Muon Chambers . . . . . . . . . . . . . . 24

2.5.1 The Central Muon Chambers ................ 24

2.5.2 The Central Muon Upgrade Chambers . . . . . . . . . . 28

2.5.3 The Central Muon Extension Chambers . . . . . . . . 28

2.6 The Trigger Systems _. . . . . . . . . . . . . . . 29

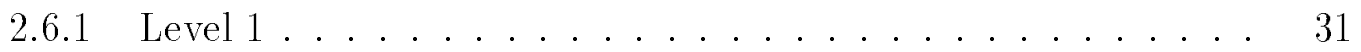


$2.6 .2 \quad$ Level $2 \ldots \ldots \ldots \ldots \ldots$. . . . . . . . . . . . . 32

2.6 .3 Level $3 \ldots \ldots \ldots \ldots \ldots \ldots$

2.7 The Data Acquisition System . . . . . . . . . . . 34

$\begin{array}{lll}3 & \text { Event Selection } & 37\end{array}$

3.1 The Data Sample . . . . . . . . . . . . . . . . . . 37

3.2 Triggers and Production . . . . . . . . . . . . . 38

$3.3 \quad W^{\prime} \rightarrow \mu \nu$ Event Identification . . . . . . . . . . . . . 39

3.4 Primary Muon Selection . . . . . . . . . . . . . . . . . 40

3.5 Missing Transverse Energy . . . . . . . . . . . . . . . 44

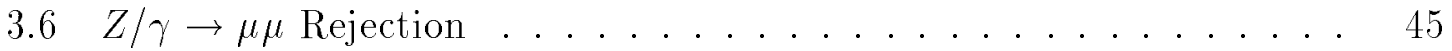

3.7 Cosmic Ray Rejection . . . . . . . . . . . . . . . 46

3.8 Final $W / W^{\prime} \rightarrow \mu \nu$ Candidate Sample . . . . . . . . . 48

4 Efficiencies $\quad 53$

4.1 Trigger Efficiencies . . . . . . . . . . . . . . . 54

4.2 Muon Selection Efficiencies . . . . . . . . . . . . . . . 55

$4.3 Z / \gamma \rightarrow \mu \mu$ and Cosmic Ray Rejection Efficiency . . . . . . . 58

4.4 Efficiency Correction for Bremsstrahlung . . . . . . . . . 60

5 Monte Carlo Simulation $\quad 63$

$5.1 W / W^{\prime} \rightarrow \mu \nu$ Event Generation $\ldots \ldots \ldots \ldots \ldots$

5.2 Detector Simulation . . . . . . . . . . . . . . . 64

5.2 .1 Underlying Event Model . . . . . . . . . . . . . . 66

5.2 .2 Detector Model . . . . . . . . . . . . . . . 73

6 Backgrounds to $W^{\prime}$ Production $\quad 78$



$6.2 W \rightarrow \tau \nu$ and $Z / \gamma \rightarrow \tau \tau \ldots \ldots \ldots \ldots \ldots \ldots$

$6.3 t \bar{t}$ Production $\ldots \ldots \ldots \ldots \ldots \ldots \ldots \ldots \ldots \ldots$

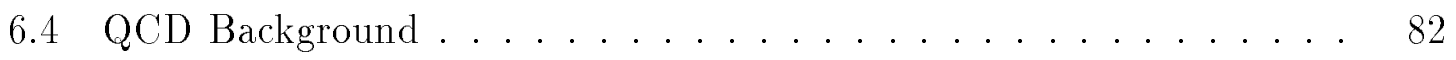

6.5 Cosmic Rays . . . . . . . . . . . . . . . . . 83 
7 Results $\quad \mathbf{8 5}$

7.1 Expected Number of $W^{\prime} \rightarrow \mu \nu$ Events . . . . . . . . . . 85

7.2 Transverse Mass Distribution Fitting . . . . . . . . . 87

7.2.1 Fitting Method ................... 87

7.2.2 Statistical Fluctuation and Smoothing . . . . . . . . 88

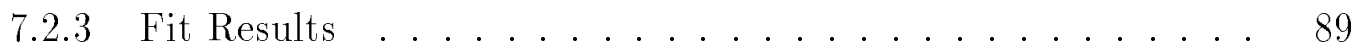

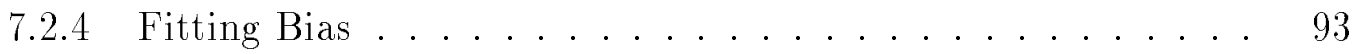

7.3 Systematic Uncertainties . . . . . . . . . . . . . 96

7.3.1 Systematic Uncertainty Calculation . . . . . . . . . . 98

7.3.2 Systematic Uncertainty Effects . . . . . . . . . . . 99

7.4 Background Contribution Estimate . . . . . . . . . . . 102

7.5 Limits ......................... 102

$\begin{array}{llr}8 & \text { Conclusions } & 108\end{array}$

8.1 Summary of Results . . . . . . . . . . . . . 108

8.2 Future Prospects . . . . . . . . . . . . . 109

A Cross-Check: Counting Experiment 11

B Individual Limits from Run 1A and Run 1B 115

C The CDF Collaboration 118 


\section{List of Figures}

$1.1 \quad W^{\prime}$-fermion-fermion coupling . . . . . . . . . . . . 7

1.2 The lowest order $W^{\prime}$ boson production Feynman diagram in $p \bar{p}$ collisions 8

1.3 The production cross section of a $W^{\prime}$ boson $\ldots \ldots \ldots$

1.4 The branching fractions for a $W^{\prime}$ boson . . . . . . . . . . . . 10

1.5 The full width of a $W^{\prime}$ boson . . . . . . . . . . . . . . 11

2.1 A schematic of the Tevatron collider complex . . . . . . . . . 14

2.2 Isometric view of the CDF detector with a quadrant cut away . . . 17

2.3 Longitudinal view of one quadrant of the CDF detector . . . . . . 17

2.4 Isometric view of one of the two SVX barrels . . . . . . . . . . . 19

2.5 CTC endplate viewed from the beam direction . . . . . . . . 21

2.6 Schematic of angular coverage by central muon chambers . . . . . . 25

2.7 Layout of the CMU chamber with respect to a central calorimeter wedge 26

2.8 Cross section of a single CMU module . . . . . . . . . . . 27

2.9 Drift tube layout for the CMX . . . . . . . . . . . 30

2.10 A schematic of the CDF data acquisition and trigger system . . . 36

3.1 The $z_{0}-z_{p v}$ distribution .................. 41

3.2 The impact parameter distributions . . . . . . . . . . . . 42

3.3 Number of hits in the CTC superlayers 3 to $8 \ldots \ldots$. . . . . . 43

3.4 The primary muon $E_{e m}$ and $E_{h a d}$ distributions . . . . . . . . 44

3.5 The primary muon Iso distributions . . . . . . . . . . . . 45

3.6 Invariant mass distributions of the events removed by the $Z / \gamma \rightarrow \mu \mu$

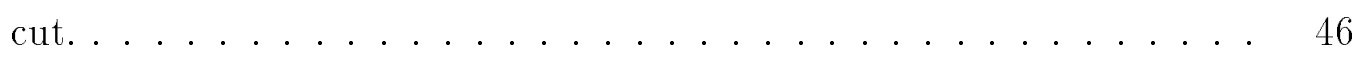

3.7 The hadron TDC, $t_{\text {had }}$, distributions . . . . . . . . . 47 
3.8 The transverse momentum distributions of the "back-to-back" tracks

3.9 The opening angle between the primary muon candidate and the muon

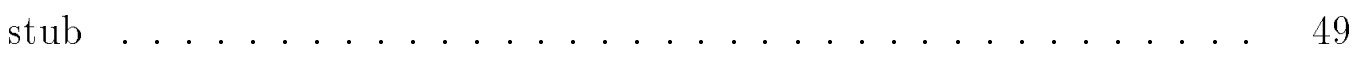

3.10 The transverse mass distributions of the $W / W^{\prime} \rightarrow \mu \nu$ event candidates 51

3.11 The event display of the highest transverse mass $W / W^{\prime} \rightarrow \mu \nu$ event

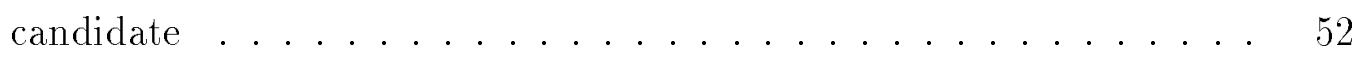

4.1 CFT efficiency as a function of $\eta \ldots \ldots . \ldots . \ldots 54$

4.2 Hadron TDC distribution of the events failed the cosmic ray rejection 59

4.3 Combined minimum ionisation cuts efficiency .......... 61

4.4 Correction factor due to final state radiation . . . . . . . . . 62

$5.1 d \sigma / d p_{T}$ and $d \sigma / d y$ distributions of $W^{+}$and $W^{\prime+}$ bosons $\ldots . . .65$

5.2 Comparisons of $u_{L}$ and $u_{T}$ distributions between $Z \rightarrow \mu \mu$ and $W \rightarrow \mu \nu \quad 67$

5.3 The invariant mass distributions of the $Z \rightarrow \mu \mu$ samples . . . . . 68

5.4 Decomposition of the observed underlying event . . . . . . . . 69

$5.5 u_{1}$ vs $p_{T}$ and $u_{2}$ vs $p_{T}$ distributions of the $Z \rightarrow \mu \mu$ sample in Run $1 \mathrm{~A} \quad 70$

$5.6 u_{1}$ vs $p_{T}$ and $u_{2}$ vs $p_{T}$ distributions of the $Z \rightarrow \mu \mu$ sample in Run 1B $\quad 70$

5.7 Comparisons between the $u_{L}$ and $u_{T}$ distributions of the $Z \rightarrow \mu \mu$ data sample and a Monte Carlo sample . . . . . . . . . . . 72

5.8 Comparisons of the underlying event between the $W / W^{\prime} \rightarrow \mu \nu$ event candidate sample and a Monte Carlo sample . . . . . . . . 72

5.9 Efficiency times acceptance of $W / W^{\prime} \rightarrow \mu \nu$ events in Run 1B . . . . . 74

5.10 The expected transverse mass distribution of the observed $W \rightarrow \mu \nu$



5.11 The expected transverse mass distributions of the $W^{\prime} \rightarrow \mu \nu$ events . . 76

5.12 The expected transverse mass distributions of observed $W^{\prime} \rightarrow \mu \nu$ events for various masses overlaid with that of $W^{\prime} \rightarrow \mu \nu$ events . . . 77

6.1 Expected transverse mass distributions of $Z / \gamma \rightarrow \mu \mu, W \rightarrow \tau \nu, Z / \gamma \rightarrow$ $\tau \tau$, and $t \bar{t}$ production in Run $1 \mathrm{~B} \ldots \ldots . \ldots 81$

6.2 The azimuthal opening angle between the muon candidate and the

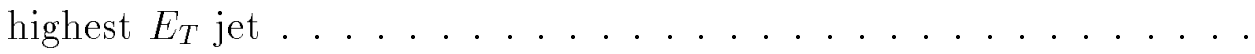


6.3 The transverse mass distribution of QCD background .......

7.1 Parametrised $M_{T}$ distributions of $W \rightarrow \mu \nu, Z / \gamma \rightarrow \mu \mu, t \bar{t} \rightarrow \mu+X$, and QCD background. . . . . . . . . . . . . 90

7.2 The $M_{T}$ distribution of the $W / W^{\prime} \rightarrow \mu \nu$ event candidates in Run $1 \mathrm{~A}$ and Run 1B with the standard model background prediction . . . . .

7.3 The $M_{T}$ distribution of the $W / W^{\prime} \rightarrow \mu \nu$ event candidates in Run 1A+1B with the standard model background prediction . . . . . . . 94

7.4 The likelihood distributions for $M_{W^{\prime}}=250 \mathrm{GeV} / c^{2}$ and $M_{W^{\prime}}=700 \mathrm{GeV} / c^{2} 95$

7.5 The distribution of the number of signal events found by the fit in background+signal pseudo-experiments . . . . . . . . . . . 97

7.6 The ratios of $\sigma \cdot \mathcal{B}\left(W^{\prime} \rightarrow \mu \nu\right)$ to $\sigma \cdot \mathcal{B}(W \rightarrow \mu \nu)$ for various parton distribution function sets . . . . . . . . . . . . 100

7.7 The systematic uncertainties $(\Delta \beta)$ as a function of $M_{W^{\prime}} \ldots \ldots$. . . 103

7.8 The likelihood distributions with uncertainty smearing . . . . . . 104

7.9 The $95 \%$ CL $\sigma \cdot \mathcal{B}\left(W^{\prime} \rightarrow \mu \nu\right)$ limit . . . . . . . . . . . . 106

7.10 The 95\% CL limit on the ratio of coupling strength times branching fraction, $\lambda^{2} \cdot \mathcal{B} \ldots \ldots \ldots \ldots 10 \ldots \ldots \ldots$

8.1 The integrated luminosity versus prospective $W^{\prime}$ mass limit . . . . . 110

A.1 The $W^{\prime}$ boson mass limits from the counting experiment . . . . . 113

B.1 The $95 \%$ CL limits on $\sigma \cdot \mathcal{B}\left(W^{\prime} \rightarrow \mu \nu\right)$ vs $M_{W^{\prime}}$ for Run $1 \mathrm{~A}$, Run $1 \mathrm{~B}$, and Run 1A and Run 1B combined .............. 116

B.2 Couplings strength times branching fraction limits for Run 1A, Run 1B, and Run 1A and Run 1B combined . . . . . . . . . 117 


\section{List of Tables}

2.1 Summary of coverage and energy resolution of the CDF calorimeters .

3.1 List of candidate events with $M_{T}>200 \mathrm{GeV} / c^{2} \ldots \ldots$. . . . 50

4.1 Trigger efficiencies in Run $1 \mathrm{~B} \ldots \ldots \ldots . \ldots . \ldots 54$

4.2 Efficiencies of selection cuts for $\mathrm{CMU} / \mathrm{P}$ muons $\ldots \ldots \ldots 56$

4.3 Efficiencies of selection cuts for CMX muons . . . . . . . . . 57

4.4 Combined efficiencies of the cuts on CMU/P and CMX muons . . . 57

4.5 Efficiencies of $\left|z_{0}-z_{p v}\right|<5 \mathrm{~cm} \mathrm{cut} \mathrm{\ldots ..........} 57$

4.6 Efficiencies of the $Z / \gamma \rightarrow \mu \mu$ rejection cut and cosmic ray rejection cuts 60

5.1 Results of the MINUIT fit to the $u_{1}$ and $u_{2} \ldots \ldots \ldots 71$

$7.1 \sigma \cdot \mathcal{B}\left(W^{\prime} \rightarrow \mu \nu\right) / \sigma \cdot \mathcal{B}(W \rightarrow \mu \nu)$ and the expected numbers of observed

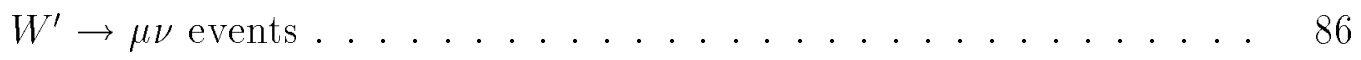

7.2 The results of the fit to the $M_{T}$ distribution of the $W / W^{\prime} \rightarrow \mu \nu$ event

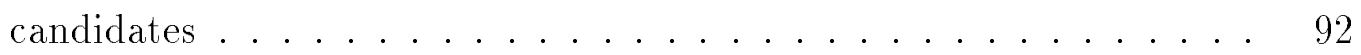

7.3 The biases in $\beta$ from the fitting method . . . . . . . . . 96

7.4 Summary of the uncertainties in the relative rates of the backgrounds. 101

7.5 The contributions from individual background components . . . . 103

7.6 The $95 \%$ confidence level limits of $\beta$ and $\sigma \cdot \mathcal{B}\left(W^{\prime} \rightarrow \mu \nu\right) \ldots \ldots 5$

A.1 The expected numbers of events for the background and the signal . . 114 


\section{Chapter 1}

\section{Introduction}

Pinky, are you pondering what I am pondering?

-from Pinky and the Brain

This thesis describes a search for a new charged heavy vector boson, generically known as a $W^{\prime}$ boson, decaying to a muon and neutrino pair. The existence of such bosons has been predicted by extensions of the paradigm describing elementary particles and the known forces, the standard model. In this chapter, theoretical motivations for the $W^{\prime}$ boson search are briefly described. The first sections discuss the standard model of the electroweak interaction and the left-right symmetric model, a particular extension of the standard model. These are followed by descriptions of the expected production and decay properties of a $W^{\prime}$ boson. Finally, the results from previous searches and the outline of this thesis are described.

\subsection{A Brief History of the Standard Model}

The development of the standard model of the electroweak weak interactions can be traced to the hypothesis of the existence of the neutrino by W. Pauli in 1933 [1]. After the neutrino postulation, E. Fermi proposed his theory of $\beta$ decay, the process by which a neutron decays to a proton, an electron, and a neutrino [2]. Subsequent discoveries of processes such as muon decay, which have a comparatively long lifetime similar to $\beta$ decay, spurred the identification of a new class of interaction, the weak 
interaction.

The parity non-conserving nature of the weak interactions was first proposed by T. D. Lee and C. N. Yang in 1956 as a way to resolve the " $\theta-\tau$ puzzle" [3], the observation that a $K$ meson could decay into two final states with opposite parity. Their hypothesis was confirmed shortly after by C. S. Wu et al. with the observation of parity violation in $\beta$ decay [4]. Also, it was shown that neutrinos are purely "lefthanded," ${ }^{1}$ which implied that parity is violated maximally in weak interactions [5]. The parity non-conserving nature of the weak interactions eventually led to the formulation of a phenomenological theory, referred to as the $V-A$ theory, that described this force as having both a vector $(V)$ and an axial-vector $(A)$ component with equal strengths but opposite sign, i.e. $V-A[6]$. The $V-A$ interaction involves only the left-handed fermions, hence the $V-A$ nature of the interaction is also referred to as left-handed.

It was understood earlier that a physical system invariant under certain symmetry transformations has associated it a set of conservation laws. The gauge symmetry (or the local symmetry) is a symmetry with respect to a space-time dependent gauge transformation and a theory with such property is known as a gauge theory. The simplest gauge theory is quantum electrodynamics. The unification of the weak interactions with electromagnetism was first suggested by J. Schwinger [7]. Later, S. L. Glashow proposed a model with an $S U(2) \times U(1)$ gauge symmetry [8], where the interaction associated with the $S U(2)$ representation acts only on the left-handed fermions. This theory lacked renormalisability because the vector boson masses were put into the theory by hand. ${ }^{2}$ A similar attempt was made by A. Salam and J. C. Ward a few years later [9]. Finally, a renormalisable theory with the Higgs mechanism of spontaneous symmetry breaking that generates the vector boson masses was proposed by S. Weinberg and A. Salam [10]. This is what became known as the "standard model" of the electroweak interaction.

Despite its enormous successes in describing electroweak phenomena, most notably

\footnotetext{
${ }^{1}$ The left-handedness refers to the negative helicity, where the helicity is defined as the projection of the spin of the particle on the direction of the motion of the particle.

${ }^{2} \mathrm{~A}$ theory is called renormalisable if infinities can be isolated and removed from the physically measurable quantities.
} 
the correct prediction of the properties of the $W$ and $Z$ bosons, the force carriers of the weak interactions, the fact that the $V-A$ structure is put into the theory by hand is quite unsatisfying.

\subsection{Left-Right Symmetric Model}

The standard model does not explain the reason why the weak interaction has a $V-A$ structure. Naturally, attempts have been made to extend the standard model to include a possible $V+A$ interaction that allows parity conservation. In these models, the observed $V-A$ nature of the weak interaction is a result of the natural suppression of the strength of the $V+A$ interaction due to heavy right-handed charged vector bosons. In this section, the mechanism by which the breakdown of a symmetry leads to masses for the vector bosons for the standard model and the left-right symmetric models is discussed.

\subsubsection{Symmetry Breaking in the Standard Model}

The Lagrangian of the gauge theory in the standard model and its extensions can be written as

$$
\mathcal{L}=\mathcal{L}_{\text {gauge }}+\mathcal{L}_{\text {fermion }}+\mathcal{L}_{\text {Yukawa }}+\mathcal{L}_{\text {Higgs }}
$$

where $\mathcal{L}_{\text {gauge }}$ is the Lagrangian for the gauge fields, $\mathcal{L}_{\text {fermion }}$ is the Lagrangian for the interactions between gauge fields and fermion fields, $\mathcal{L}_{Y \text { ukawa }}$ is the Lagrangian for the fermion fields and Higgs fields interaction, which gives rise to the fermion masses, and $\mathcal{L}_{\text {Higgs }}$ is the Lagrangian for the Higgs fields. The Higgs term is written as

$$
\mathcal{L}_{\text {Higgs }}=\left|D_{\mu} \Phi\right|^{2}-V(\Phi)
$$

where $D_{\mu}$ is a covariant derivative, $\Phi$ is the Higgs field, and $V(\Phi)$ is the Higgs potential. The covariant derivative $D_{\mu}$ is written as

$$
D_{\mu}=\partial_{\mu}-\frac{i g}{2} \tau \cdot \mathbf{A}_{\mu}-\frac{i g^{\prime}}{2} B_{\mu}
$$


where $g$ and $g^{\prime}$ are the coupling constants associated with the $S U(2)_{L}$ and the $U(1)_{Y}$ components of the electroweak interactions, respectively, $\mathbf{A}_{\mu}$ and $B_{\mu}$ are the gauge field triplets of $S U(2)_{L}$ and the singlet of $U(1)_{Y}$, respectively, and $\tau / 2$ are the $S U(2)$ generators, the Pauli spin matrices.

The Higgs field is a doublet of a complex field

$$
\Phi=\left(\begin{array}{l}
\phi^{+} \\
\phi^{0}
\end{array}\right)
$$

and the Higgs potential $V(\Phi)$ is chosen to have a minimum

$$
\Phi_{0}=\left(\begin{array}{c}
0 \\
v / \sqrt{2}
\end{array}\right)
$$

where $v$ is a real number denoted as the vacuum expectation value for the Higgs field. This non-vanishing vacuum expectation value implies that the vacuum is usually not invariant under the $S U(2) \times U(1)$ symmetry, thus the symmetry is broken “spontaneously". By replacing $\Phi$ with $\Phi^{\prime}+\Phi_{0}$, one gets a gauge field mass term from $\mathcal{L}_{\text {Higgs }}$

$$
\frac{v^{2}}{8}\left[g^{2} W^{+}{ }_{\mu} W^{-\mu}+\left(g A_{3 \mu}-g^{\prime} B_{\mu}\right)^{2}\right]
$$

where $W^{ \pm}=\left(A_{1} \mp i A_{2}\right) / \sqrt{2}$ are the $W$ boson fields. The fields in the second term are identified as the $Z$ boson field

$$
Z_{\mu}=\frac{1}{\sqrt{g^{2}+g^{\prime 2}}}\left(g A_{3 \mu}-g^{\prime} B_{\mu}\right) .
$$

Then the mass term becomes

$$
\frac{1}{2} M_{W}^{2} W^{+}{ }_{\mu} W^{-\mu}+\frac{1}{2} M_{Z}^{2} Z_{\mu} Z^{\mu}
$$

where $M_{W}=\frac{1}{2} v g$ and $M_{Z}=\frac{1}{2} v \sqrt{g^{2}+g^{\prime 2}}$. The photon field is orthogonal to $Z_{\mu}$ and is massless. The weak mixing angle (or Weinberg angle), $\theta_{W}$, is defined as

$$
\sin ^{2} \theta_{W} \equiv \frac{g^{\prime 2}}{g^{\prime 2}+g^{2}}=1-\left(\frac{M_{W}}{M_{Z}}\right)^{2}
$$


which is the unification condition for the electromagnetic and weak interactions.

The masses of the gauge bosons therefore depend on three parameters; $g, g^{\prime}$, and $v$. These parameters can be inferred from measurements of $G_{F}$, the Fermi coupling constant, $\alpha$, the electromagnetic coupling constant, and $\sin ^{2} \theta_{W}$. Therefore, the masses of $W$ and $Z$ bosons were predicted reasonably well before their discoveries in $1983[11,12,13]$.

\subsubsection{Symmetry Breaking in Left-Right Symmetric Model}

Within the framework of a gauge theory, the idea of the left-right symmetry in the electroweak interaction was realised as the left-right symmetric model based on the gauge group $S U(2)_{L} \times S U(2)_{R} \times U(1)_{B-L}[14]$. This model has an appealing feature: the $U(1)$ generator, $B-L$, baryon number minus lepton number, has a physical significance and suggests a deeper symmetry between quarks and leptons. ${ }^{3}$

The Higgs fields required to break the symmetry are not unique in the left-right symmetric model. The simplest form of the left-right symmetric model requires three Higgs field multiplets, which are usually denoted as $\Delta_{L}, \Delta_{R}$, and $\Phi$, to break the $S U(2)_{L} \times S U(2)_{R} \times U(1)_{B-L}$ down to the $U(1)_{E M}$. Here, $\Delta_{R}\left(\Delta_{L}\right)$ controls the right(left)-handed sector of the theory and $\Phi$ serves to connect left-and right-handed fermionic multiplets and to give the fermions mass after the symmetry breaking.

The $S U(2)_{L} \times S U(2)_{R} \times U(1)_{B-L}$ symmetry is broken down in the following pattern: $S U(2)_{L} \times S U(2)_{R} \times U(1)_{B-L} \rightarrow S U(2)_{L} \times U(1)_{Y} \rightarrow U(1)_{E M}$. The first stage of the symmetry breaking gives the masses to $W_{R}$ and $Z_{R}$ bosons, which are bosons in the right-handed sector corresponding to the standard model $W$ and $Z$ bosons, respectively. The subsequent stage of the symmetry breaking gives the masses to the $W_{L}$ and $Z_{L}$ bosons, which are equivalent to the standard model $W$ and $Z$ bosons, respectively. Because the symmetry breaking pattern dictates that the vacuum expectation value for $\Delta_{R}$, which contribute to the $W_{R}$ boson mass, be greater than those for $\Delta_{L}$ and $\Phi$, which contribute to the $W_{L}$ boson mass, the $W_{R}$ boson is expected to be heavier than the $W_{L}$ boson. The observable (physical) states of charged bosons

\footnotetext{
${ }^{3}$ The weak hypercharge $Y$ in $U(1)_{Y}$ of the standard model is introduced to assign the correct electric charge.
} 
are expected to be a linear combination of $W_{L}$ and $W_{R}$ bosons. However, the mixing angle should be small and the $W_{L}$ and $W_{R}$ bosons approximately coincide with their respective physical states. ${ }^{4}$

Besides the additional vector bosons and multiple Higgs bosons, an important aspect of the left-right symmetric model is the existence of non-zero mass neutrinos. Because the right-handed neutrinos exist in the left-right symmetric models, the neutrinos obtain masses through the symmetry breaking. In the case that neutrinos are Majorana particles (i.e. particles that are antiparticles of themselves), the lefthanded neutrino and the right-handed neutrino can have distinctive masses and the right-handed neutrino can be heavy enough to forbid $V+A$ interactions involving neutrinos [15].

Although the left-right symmetry based on an $S U(2)$ group was motivated by "philosophical" considerations as it is aesthetically more gratifying, one can also obtain $S U(2)_{L} \times S U(2)_{R}$ from theories based on higher gauge symmetries. For example, in a grand unified theory based on $S O(10), S O(10)$ may break down to $S U(2)_{L} \times S U(2)_{R} \times U(1)_{B-L}$ as an intermediate stage.

\subsection{Production and Decay of a $W^{\prime}$ Boson}

The production of a $W^{\prime}$ boson in a $p \bar{p}$ collision is similar to that of a $W$ boson. ${ }^{5}$ The Lorentz-invariance and renormalisability of the vector boson coupling requires the matrix element for the $W^{\prime}$-fermion-fermion coupling shown in Figure 1.1 to have the form of

$$
\mathcal{M}=-\frac{i g_{R}}{\sqrt{2}} W_{\alpha}^{\prime} \bar{\psi}_{i} \gamma^{\alpha}\left(a+b \gamma_{5}\right) \psi_{j} U_{R_{i j}}
$$

where $g_{R}$ is the coupling constant for the right-handed sector, $a$ and $b$ are constants, and $U_{R_{i j}}$ is the Cabibbo-Kobayashi-Maskawa (CKM) matrix element connecting fermions $i$ and $j$ for the right-handed sector. It is assumed that $g_{R}$ is the same as $g\left(=g_{L}\right)$ of the standard model and the CKM matrix for the right-handed

\footnotetext{
${ }^{4}$ The mixing angle is assumed to be zero throughout this thesis and, thus, $W_{R}$ and $W_{L}$ bosons are regarded as physical bosons.

${ }^{5}$ Unless noted otherwise, from here on $W_{R}$ and $W_{L}$ bosons are referred to as $W^{\prime}$ and $W$ bosons, respectively.
} 


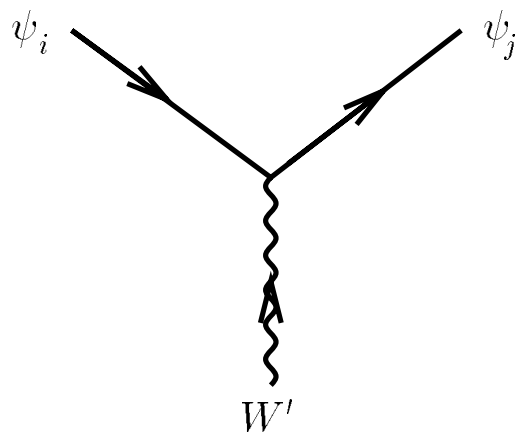

Figure 1.1: $W^{\prime}$-fermion-fermion coupling.

sector is the same as that of the standard model. ${ }^{6}$

The partial width of a $W^{\prime}$ boson to a quark pair $q_{i}$ and $\bar{q}_{j}$ is

$$
\Gamma_{i j}=\frac{a^{2}+b^{2}}{2} \frac{N_{c} G_{F} M_{W}^{2} M_{W^{\prime}}}{6 \sqrt{2}}\left|U_{i j}\right|^{2}
$$

where $N_{c}$ is the color factor, which is three for the standard model as well as the manifest left-right symmetric model. One can define $\lambda$ as

$$
\lambda^{2} \equiv \frac{a^{2}+b^{2}}{2}
$$

where $a=1$ and $b=-1$, i.e. $\lambda^{2}=1$, for the standard model. For the left-right symmetric model where the coupling strength for the right-handed sector is the same as that of the left-handed sector, $\lambda^{2}=1 .^{7}$ This case will be referred to as "the standard model strength" couplings from here on.

The lowest order production mechanism for a $W^{\prime}$ boson in a $p \bar{p}$ collision is quark and anti-quark annihilation as shown in Figure 1.2. The differential production cross section for a $W^{\prime}$ boson is

$$
\frac{d \sigma}{d y}\left(p \bar{p} \rightarrow W^{\prime}+X\right)=\frac{4 \pi^{2}}{3 M_{W^{\prime}}^{3}} \sum_{i, j} x_{i} x_{j} q_{i}^{a}\left(x_{i}, M_{W^{\prime}}^{2}\right) q_{j}^{b}\left(x_{j}, M_{W^{\prime}}^{2}\right) \Gamma_{i j}
$$

\footnotetext{
${ }^{6}$ The left-right symmetric model with the identical CKM matrices for both left- and right-handed sectors is referred to as the manifest left-right symmetric model.

${ }^{7}$ The $\lambda^{2}$ can be absorbed into the definition of $g_{R}$, but it is used as a measure of the coupling strength for the left-right symmetric model in the literature.
} 




Figure 1.2: The lowest order $W^{\prime}$ boson production Feynman diagram in $p \bar{p}$ collisions. A $W^{\prime}$ boson is produced from a quark-antiquark annihilation and decays into a pair of fermions.

where $q_{i}^{a}\left(x_{i}, M_{W^{\prime}}^{2}\right)$ is a parton distribution, or probability distribution, of a quark type $i$ with a momentum fraction $x_{i}$ calculated at the scale $M_{W}^{2}$, in a hadron type $a$. The rapidity, $y$, of the $W^{\prime}$ boson is related to the momentum fractions, $x_{1}$ and $x_{2}$, of quarks by

$$
x_{1,2}=\frac{M_{W^{\prime}}}{\sqrt{s}} e^{ \pm y}
$$

where $\sqrt{s}$ is the center-of-beam energy of the $p \bar{p}$ system.

The total production cross section of a $W^{\prime}$ boson is shown in Figure 1.3 at $\sqrt{s}=1.8 \mathrm{TeV}$ assuming the standard strength couplings and the CKM matrix. The cross section was calculated with CTEQ4A1 parton distribution function set [16], as discussed in Section 5.1.

The measurement reported in this thesis is an upper limit on the production cross section of a $W^{\prime}$ boson times its branching fraction to a muon and neutrino pair for a given $W^{\prime}$ boson mass. In order to convert it into a limit on the $W^{\prime}$ boson mass, the branching fraction has to be calculated. Figure 1.4 shows the branching fraction of possible decay channels of a $W^{\prime}$ boson for standard strength couplings, which includes the $W^{\prime} \rightarrow t b$ decay channel with a top quark mass at $176 \mathrm{GeV} / c^{2}$ [17]. The 


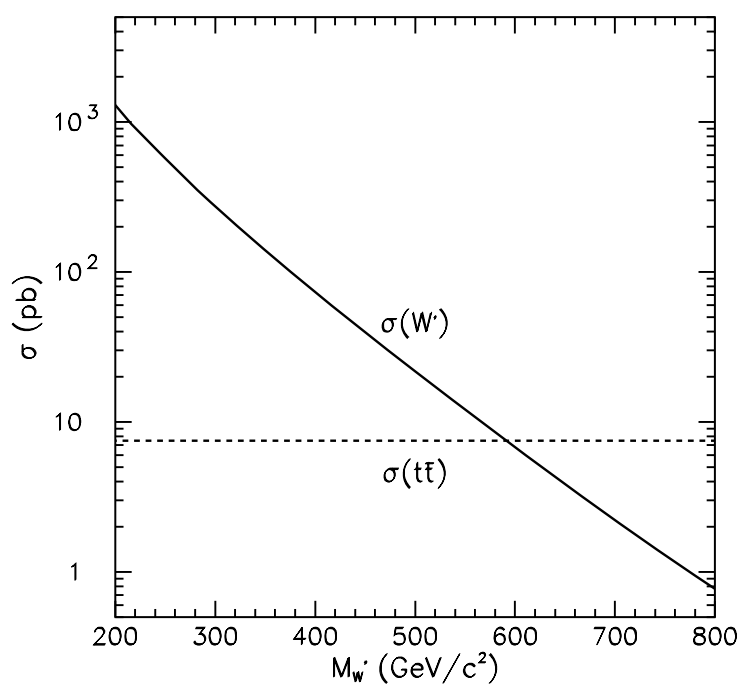

Figure 1.3: The production cross section of a $W^{\prime}$ boson for standard strength couplings in $p \bar{p}$ collisions at $\sqrt{s}=1.8 \mathrm{TeV}$. The $t \bar{t}$ production cross section $\left(M_{t}=176 \mathrm{GeV} / c^{2}\right)$ is also shown for comparison [17].

branching fraction for the individual lepton decay channel approaches $1 / 12$ as the $W^{\prime}$ boson becomes heavier. In calculating the branching fractions, it was assumed that the boson pair decay channel, $W^{\prime} \rightarrow W Z$, is very small and can be ignored. In fact, the $W^{\prime} \rightarrow W Z$ decay channel is substantially suppressed in extended gauge models and its branching fraction is independent of a $W^{\prime}$ boson mass [18]. The total width of the $W^{\prime}$ boson used in this analysis was determined assuming the standard strength couplings, and also accounted for the top quark contribution. Figure 1.5 shows this total width as a function of the $W^{\prime}$ boson mass.

The choice of the $W^{\prime} \rightarrow \mu \nu$ decay channel in this thesis was motivated by the large size of data available compared to that for the previous search with the same decay channel (see Section 1.4). With $\sim 25$ times more data, this search can extend the limit by a large amount.

There are three different $W^{\prime} \rightarrow \mu \nu$ decay scenarios, depending on the right-handed muon neutrino mass: 1) The neutrino is light and stable. The $W^{\prime} \rightarrow \mu \nu$ decay is similar to the $W \rightarrow \mu \nu$ decay. 2) The neutrino is heavy but lighter than a $W^{\prime}$ boson and is most likely to decay inside the detector volume. In this case, the "neutrino signature" in the decay is lost. 3) The neutrino is heavier than a $W^{\prime}$ boson. All lepton 


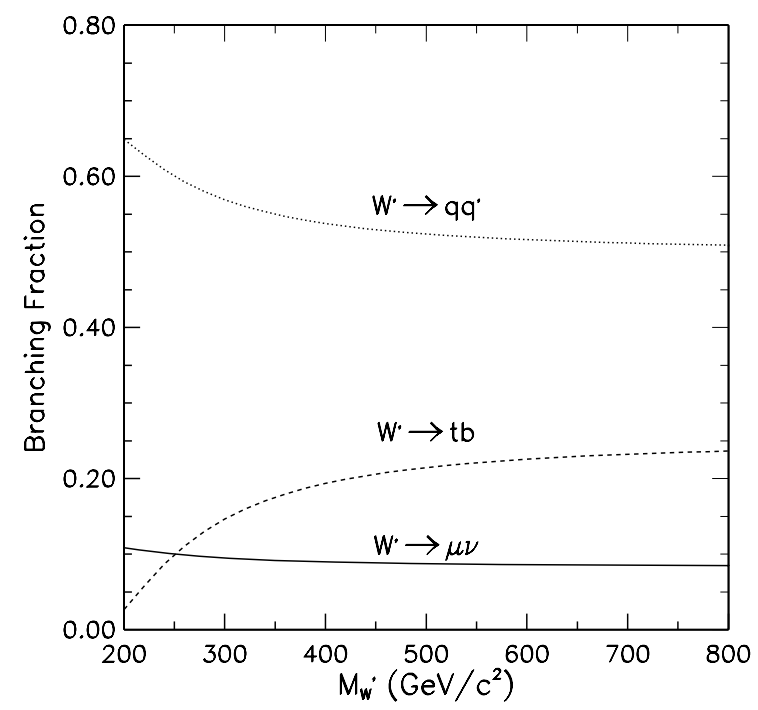

Figure 1.4: The branching fractions for a $W^{\prime}$ boson to various decay channels as a function of the $W^{\prime}$ boson mass. The decay channel $W^{\prime} \rightarrow q q^{\prime}$ does not include the $W^{\prime} \rightarrow t b$ channel.

decay channels are completely closed if neutrinos of other lepton families are as heavy as the muon neutrino. In this case, a $W^{\prime}$ boson predominantly decays hadronically. In this analysis, a $W^{\prime}$ boson corresponding to case 1 ) is searched for.

\subsection{Previous Searches}

A $W^{\prime}$ boson has been searched for directly by looking for the decay products of the boson. The DØ collaboration has set a lower mass limit by searching for $W^{\prime} \rightarrow e \nu$ events and concluded that $M_{W^{\prime}}>720 \mathrm{GeV} / c^{2}$ for $M_{W^{\prime}} \gg M_{\nu_{R}}$ and $M_{W^{\prime}}>650 \mathrm{GeV} / c^{2}$ for $1 / 2 M_{W^{\prime}}<M_{\nu_{R}}$ at $95 \%$ confidence level, where the $\nu_{R}$ decays into an electron and two jets [19]. For the $W^{\prime} \rightarrow \mu \nu$ decay channel, a lower mass limit of $M_{W^{\prime}}>435 \mathrm{GeV} / c^{2}$ at $95 \%$ confidence level has been set by the CDF collaboration [20]. The dijet decay channel was used to set limits of $M_{W^{\prime}}>261 \mathrm{GeV} / c^{2}$ at $90 \%$ confidence level at UA2 and $300<M_{W^{\prime}}<420 \mathrm{GeV} / c^{2}$ at $95 \%$ confidence level at CDF [21, 22]. These direct searches assumed the manifest left-right symmetric model.

In addition to direct searches, limits on $W_{R}$ bosons have been placed indirectly from the absence of $V+A$ currents in low energy phenomena. Using a polarized $\mu^{+}$ 


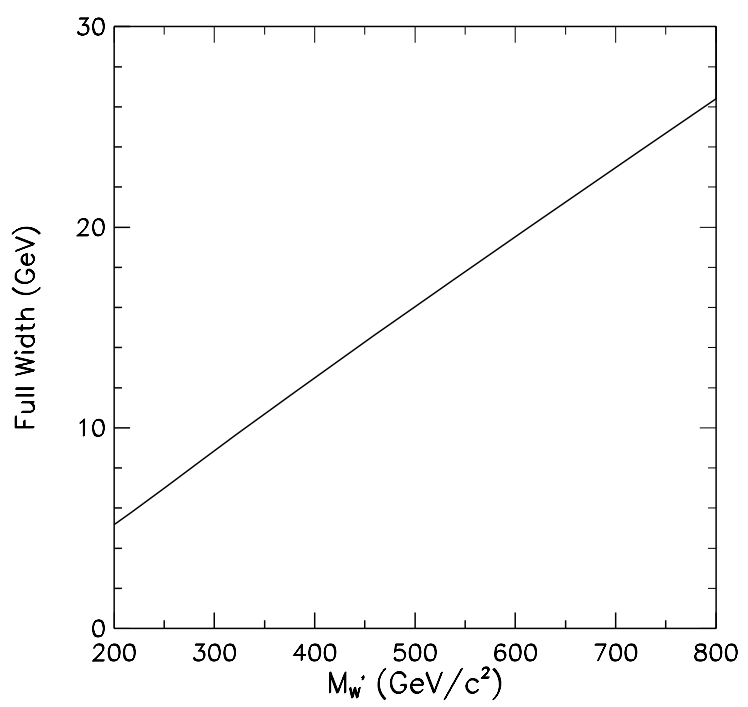

Figure 1.5: The full width of a $W^{\prime}$ boson as a function of its mass.

beam at TRIUMF $^{8}$ the relative $e^{+}$rate at the momentum end point in a direction opposite to the $\mu^{+}$spin was measured. This yielded the mass limit of $406 \mathrm{GeV} / \mathrm{c}^{2}$ at $90 \%$ confidence level assuming a massless right-handed neutrino [23]. Also, using muons produced in $K^{+} \rightarrow \mu^{+} \nu$ decay that subsequently decayed into $e^{+} \nu_{e} \bar{\nu}_{\mu}$, a search has been made for $V+A$ currents at KEK. ${ }^{9}$ This yielded a limit varying from $M_{W_{R}}>303 \mathrm{GeV} / c^{2}$ to $M_{W_{R}}>653 \mathrm{GeV} / c^{2}$ at $90 \%$ confidence level, depending on the assumption made on the right-handed sector [24].

Theoretical studies have attempted to constrain $M_{W_{R}}$ using various experimental and theoretical inputs. For the manifest left-right symmetric model, $M_{W_{R}}$ was constrained to be $M_{W_{R}} \gtrsim 0.3-1.4 \mathrm{TeV}[25]$.

The mass $M_{W_{R}}$ has also been constrained from the observation of neutrino flux from a supernova. If a right-handed neutrino is light $\left(M_{\nu_{R}} \leq 10 \mathrm{MeV} / c^{2}\right)$ and it has a charged-current coupling, it can be produced inside the core of a supernova via the process $e_{R}^{-} p \rightarrow \nu_{R} n$. By observing the neutrino flux from the supernova $1987 \mathrm{~A}$, one can exclude $3.7-6.7 \leq M_{W_{R}} / M_{W_{L}} \leq 280-500$ in the absence of $W_{L}-W_{R}$ mixing [26].

Although these searches have all produced null results, the recent results from

\footnotetext{
${ }^{8}$ Tri-University Meson Facility.

${ }^{9}$ KEK stands for Ko Enèrugi gashōkki Kenkūshō or literally means the high energy accelerator laboratory.
} 
the Super-Kamiokande experiment have shown evidence that neutrinos are not massless [27]. The non-zero neutrino mass, as mentioned earlier, is one of the consequences of the left-right symmetric models, suggesting the possibility of the existence of a $W^{\prime}$ boson.

\subsection{Outline of Dissertation}

This dissertation describes a new search for a $W^{\prime}$ boson decaying to a muon and neutrino pair, extending the sensitivity of the earlier $W^{\prime} \rightarrow \mu \nu$ search. The data used for this analysis come from a data sample of $p \bar{p}$ collisions recorded by the Collider Detector at Fermilab over a three-year period. A new technique is used to extract the limits on producing such events, in which the expected yields of $W^{\prime} \rightarrow \mu \nu$ events were normalised with respect to the expected yields of $W \rightarrow \mu \nu$ events.

The remainder of this thesis is organized as follows. Chapter 2 gives an overview of the experimental apparatus, the Tevatron Collider and the CDF detector. Chapter 3 describes $W^{\prime} \rightarrow \mu \nu$ event signatures and event selection criteria followed by the efficiency calculation of the event selection criteria in chapter 4. Chapter 5 describes the Monte Carlo simulations to generate $W$ and $W^{\prime}$ events and to model the detector response. Chapter 6 describes the estimation of backgrounds in the event sample. In chapter 7 the search method, the search results, and the technique used to extract the limits are detailed. The conclusions of this study are discussed in Chapter 8. 


\section{Chapter 2}

\section{The Experimental Apparatus}

The apparatus used in this experiment consists of two major parts. The Tevatron, a proton-antiproton collider, provides the counter-rotating proton and antiproton beams that collide with a center-of-mass energy $(\sqrt{s})$ of $1.8 \mathrm{TeV}$. The Collider Detector at Fermilab (CDF), a general purpose particle detector designed to study $p \bar{p}$ collisions at the Tevatron, detects and records these collisions.

In this chapter, the Tevatron is briefly described followed by detailed description of the CDF detector.

\subsection{The Tevatron Accelerator}

Figure 2.1 shows the schematics of the Tevatron collider complex. In order to obtain $900 \mathrm{GeV}$ counter-rotating proton and antiproton beams, protons and antiprotons are accelerated in several stages.

First, extra electrons are added to hydrogen gas to make $H^{-}$ions. These ions are then accelerated to an energy of $750 \mathrm{keV}$ by a Cockroft-Walton accelerator. The ions are then injected into a $175 \mathrm{~m}$ long linear accelerator (linac) that accelerates the ions to $400 \mathrm{MeV}$, bunching them at the same time. The ions are passed through a thin carbon foil that strips off their electrons, resulting in a proton beam. The protons are then injected into a circular booster of $75 \mathrm{~m}$ radius and emerge with an energy of $8 \mathrm{GeV}$.

For antiproton production, protons are injected into the Main Ring and are 


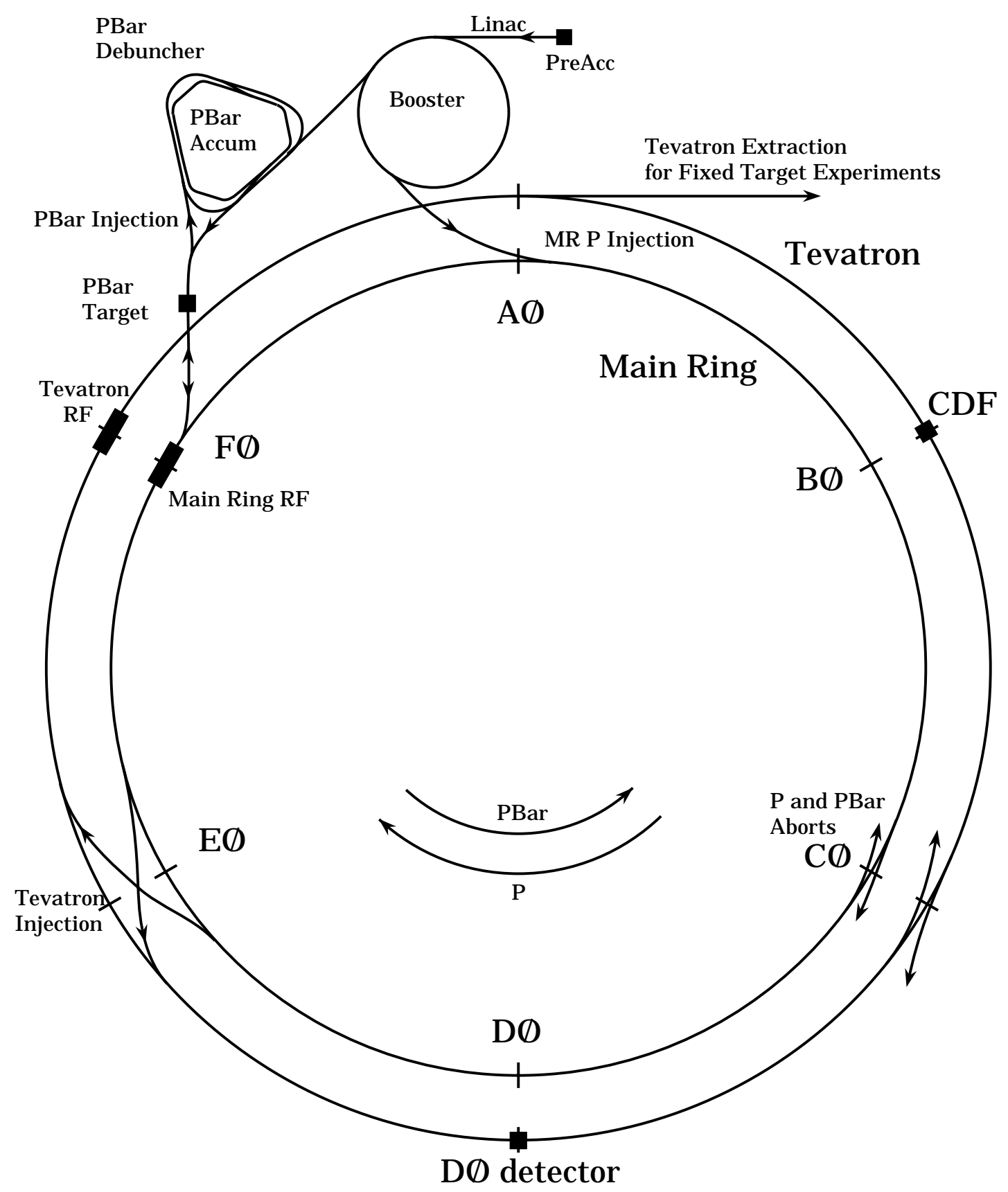

Figure 2.1: A schematic of the Tevatron collider complex (not drawn to scale). 
boosted to an energy of $120 \mathrm{GeV}$. The Main Ring is a proton synchrotron $6.3 \mathrm{~km}$ in circumference. The protons are kept in a circular orbit using 1000 conventional magnets that bend and focus the beam. The protons are then extracted and directed to an antiproton production target, consisting of nickel. Negatively charged particles coming out from the target as a result of the collisions of protons with the target are passed through a bending magnet. Only negatively charged particles with an energy of $8 \mathrm{GeV}$ are collected and transported to the Debuncher, where the particles other than antiprotons are removed after a few cycles. The Debuncher also reduces the momentum spread of the beam and stochastically "cools" the beam. The Debuncher has sensors that measure the momentum spread of the beam and send a signal to a "kicker" on the other side of the Debuncher that gives corrective "kicks" to the beam to reduce the spread. After cooling, the antiproton beam is injected into the Accumulator where the beam is cooled even further and stored for later injection into the Main Ring.

When there are enough antiprotons (typically $5 \times 10^{11}$ antiprotons) in the Accumulator, protons are no longer directed to the antiproton target but are accelerated to $150 \mathrm{GeV}$ in the Main Ring. The proton bunches are coalesced further and then injected into the Tevatron. The Tevatron is a proton synchrotron with $\sim 1000 \mathrm{su-}$ perconducting magnets housed in the same tunnel as the Main Ring. The process is repeated five more times resulting in six bunches of protons spaced symmetrically in the Tevatron. Then antiprotons are injected into the Main Ring from the Accumulator and are accelerated to $150 \mathrm{GeV}$. Antiproton bunches are also coalesced into a single bunch and the bunch is injected into the Tevatron rotating opposite to the direction of the protons. This process is repeated five more times. The proton and antiproton beams are then accelerated to $900 \mathrm{GeV}$ resulting in counter-rotating proton-antiproton beams with six equally spaced bunches each. The proton and antiproton bunches pass through each other at six interaction regions in the Tevatron, labelled $\mathrm{A} \varnothing$ through $\mathrm{F} \varnothing$. The $\mathrm{CDF}$ detector is located at the $\mathrm{B} \varnothing$ interaction region. A second detector $\mathrm{D} \varnothing$ is located at the $\mathrm{D} \varnothing$ interaction region. A proton-antiproton collision has a center-of-mass energy $(\sqrt{s})$ of $1.8 \mathrm{TeV}$. 


\subsection{Overview of the CDF Detector}

The Collider Detector at Fermilab (CDF) is a general-purpose detector designed to study the results of $p \bar{p}$ collisions at $\sqrt{s}=1.8 \mathrm{TeV}$. It is almost cylindrically symmetric with respect to the beam line and also is forward-backward symmetric along the beam line with the center of the detector located at the nominal $p \bar{p}$ collision point. It consists of several layers of different detector subsystems that are designed to measure the various aspects of particles produced in the collisions. An isometric view of the detector is shown in Figure 2.2.

Figure 2.3 shows a schematic view of a quadrant of the CDF detector along with the coordinate system, $(x, y, z)$, employed in this study. The $z$-direction is defined to be the proton beam direction and the $x$-direction is defined to be pointing out of the Tevatron ring. The polar angle, $\theta$, and the azimuthal angle, $\phi$, are defined with respect to the $z$ direction. One useful variable to describe the $p \bar{p}$ collisions is pseudorapidity, $\eta$, that is defined as

$$
\eta \equiv-\ln \left(\tan \frac{\theta}{2}\right)
$$

with $\theta$ measured from either the nominal $p \bar{p}$ collision point, i.e. $z=0$, or the actual $p \bar{p}$ collision point.

The nominal $p \bar{p}$ collision point is surrounded by a beryllium beam pipe $3.8 \mathrm{~cm}$ in diameter and tracking detectors that measure the position of the collisions and the momenta of the charged particles produced in each collision. They are immersed inside a $1.4 \mathrm{~T}$ magnetic field generated by a solenoid $5 \mathrm{~m}$ long and $3 \mathrm{~m}$ in diameter consisting of 1164 turns of $\mathrm{NbTi} / \mathrm{Cu}$ superconductor. The tracking system is described in detail in Section 2.3.

The tracking detectors are surrounded by the calorimeter systems that measure the electromagnetic and hadronic energy flow from the $p \bar{p}$ collision points. Behind the calorimeter systems are drift chambers comprising the muon systems that detect the muons that penetrated calorimeters and other materials. The calorimeter systems and the muon systems are described in detail in Sections 2.4 and 2.5, respectively. 


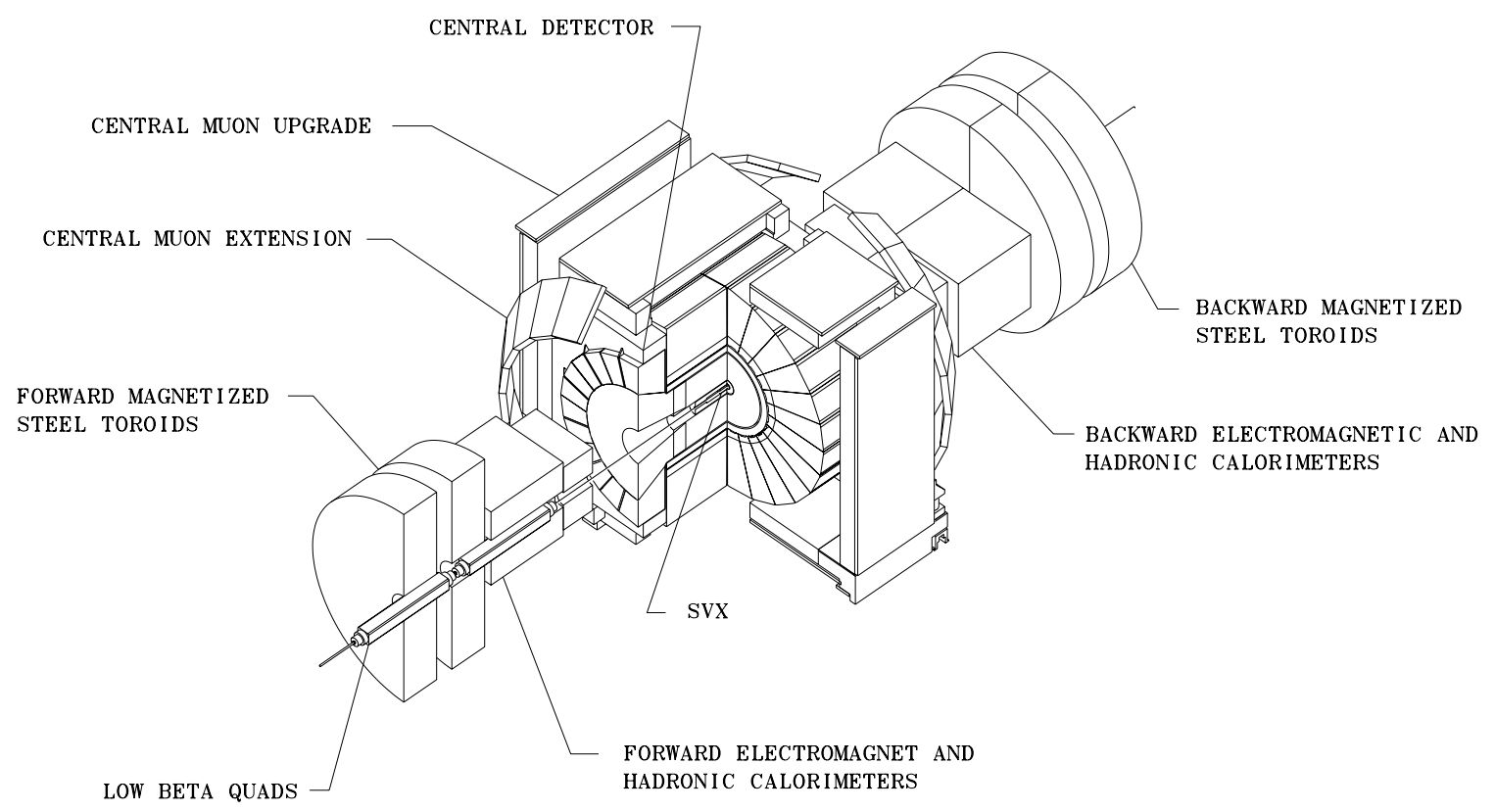

Figure 2.2: Isometric view of the CDF detector with a quadrant cut away to expose the inner tracking detectors.

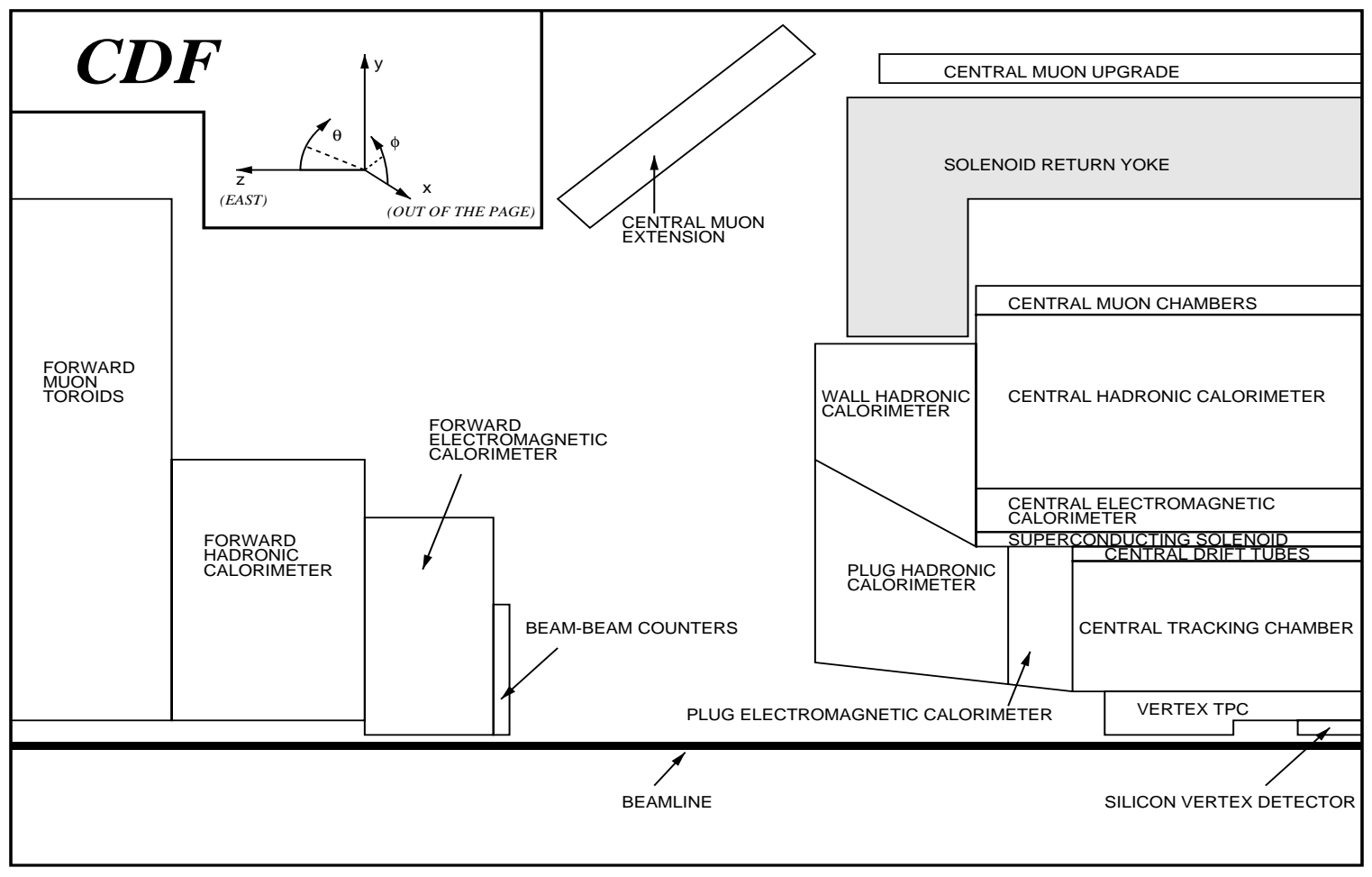

Figure 2.3: Longitudinal view of one quadrant of the CDF detector. The coordinate definitions are shown in the inset. 


\subsection{The Tracking Detectors}

The CDF tracking system consists of the silicon vertex detector (SVX), the vertex time projection drift chamber (VTX), the central tracking chamber (CTC), and the central drift tube array (CDT).

The SVX [29, 30] is designed to detect charged particles close to the interaction points enabling the precise measurement of the proton-antiproton collision point as well as secondary vertices that result from $b$-quark decays. The SVX information is also used to determine the momenta of charged particles in conjunction with the CTC. Originally the SVX was installed in 1992 and was replaced by an improved version, the $\mathrm{SVX}^{\prime}$, in $1993 .^{1}$

The SVX consists of two identical cylindrical modules. An isometric view of one of the SVX modules is shown in Figure 2.4. Each module has four layers of silicon microstrip detectors concentrically arranged from $3.0 \mathrm{~cm}$ to $7.9 \mathrm{~cm}$ away from the beam line. For the SVX', the inner most layer is located $2.9 \mathrm{~cm}$ from the beam line. The SVX active region is $51 \mathrm{~cm}$ long, with a $2.15 \mathrm{~cm}$ gap at $z=0$ where two modules are joined.

Each layer consists of twelve "ladder" modules spanning about $30^{\circ}$ in $\phi$ and aligned parallel to the $z$ axis. The adjacent ladder modules overlap each other slightly to ensure that the detector provides complete coverage in $\phi$ to charged particles, with the exception of the inner most layer of the SVX, which has $1.26^{\circ}$ gaps in $\phi$ between the adjacent ladder modules. Each ladder module is made up of three single-sided silicon microstrip detectors that are $8.5 \mathrm{~cm}$ long and $\sim 300 \mu \mathrm{m}$ thick with the strips running in the $z$ direction. These three detectors are electrically connected with "microbonds". The silicon strips have a pitch of $60 \mu \mathrm{m}$ for three inner layers and $55 \mu \mathrm{m}$ for the fourth layer. The SVX has a total of 46080 readout channels. The average spatial resolutions are $13 \mu \mathrm{m}$ for the SVX and $11.6 \mu \mathrm{m}$ for the SVX', and the resulting impact parameter resolutions for the high transverse momentum track are $17 \mu \mathrm{m}$ for the SVX and $13 \mu \mathrm{m}$ for the SVX'. In the analysis presented here, the SVX is used to determine the position of the beam line and the particle track positions.

\footnotetext{
${ }^{1}$ The SVX will refer to both the SVX and SVX', unless noted otherwise.
} 


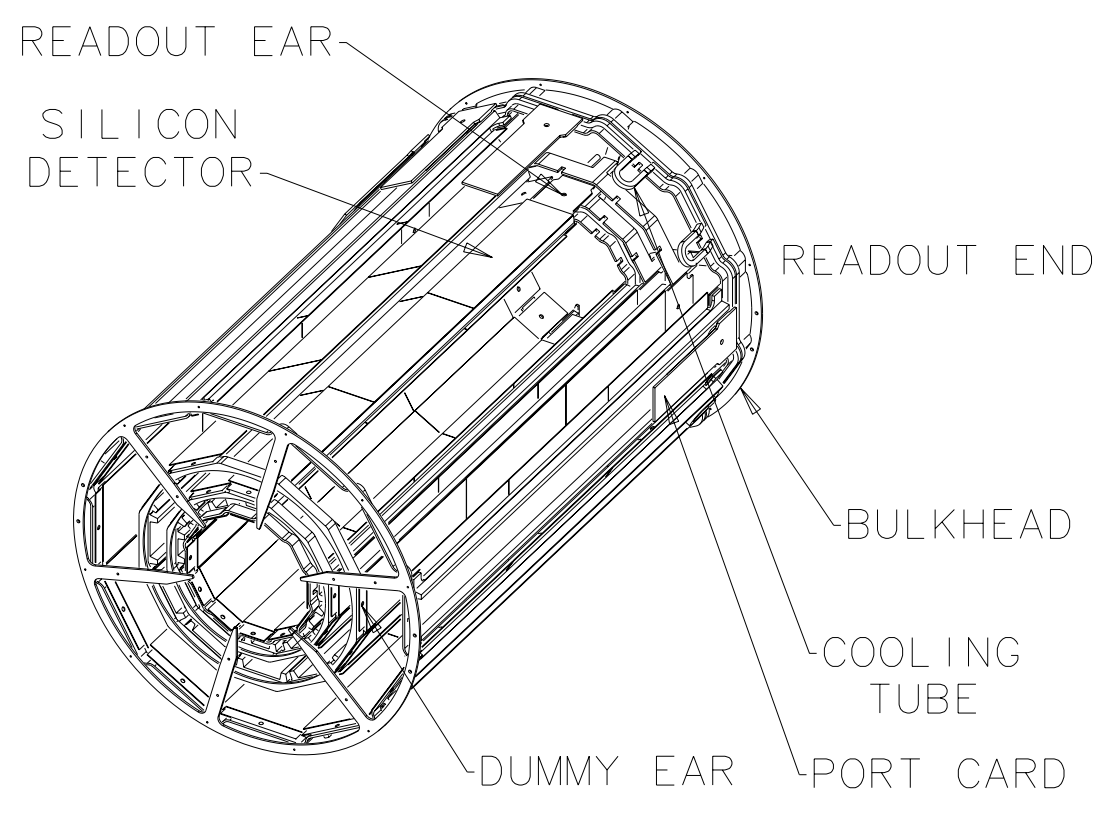

Figure 2.4: Isometric view of one of the two SVX barrels. The barrels are joined at the dummy-ear sides with an effective gap of $2.15 \mathrm{~cm}$ between active regions.

The SVX and the beam pipe are surrounded by a set of vertex time projection drift chambers (VTX) [31]. The VTX measures the $r-z$ position of particle tracks and is used to determine the $z$ position of the $p \bar{p}$ interaction vertex with a resolution of approximately $2 \mathrm{~mm}$. The VTX consists of 284 -cm long octagonal time projection chamber modules with outer radii of $22 \mathrm{~cm}$ and covers $280 \mathrm{~cm}$ in $z$ direction. Each chamber module has a high voltage grid that divides it into two equal length drift regions.

The Central Tracking Chamber (CTC) [32] is a $3.2 \mathrm{~m}$ long cylindrical drift chamber with inner and outer radii of $31 \mathrm{~cm}$ and $132 \mathrm{~cm}$, respectively, and mounted outside the VTX. It consists of 6156 sense wires grouped into 84 layers arranged in nine superlayers labelled 0 to 8 . Figure 2.5 shows one of the CTC endplates, which illustrates the wire mount slots. In a given superlayer, the sense wires define cells that are electrically separated. There are five axial superlayers $(0,2,4,6$, and 8) whose wires are parallel to the beam line, with each consisting of 12 layers of sense wires. There are four stereo superlayers $(1,3,5$, and 7$)$ whose wires are canted $\pm 3^{\circ}$ with respect to the beam line providing $r-z$ information. The stereo superlayers have 6 layers of 
sense wires. There are $30,42,48,60,72,84,96,108$, and 120 cells from superlayer 0 to 8 , respectively.

Each cell consists of sense wires surrounded by field wires and and potential wires in between each sense wire. The sense wires are made of $40 \mu \mathrm{m}$ diameter gold-plated tungsten. The field wire voltages control the strength of the drift field whereas the potential wire voltages control the gas gain on the sense wires. The cells are arranged such that the electric field inside the cell is approximately $45^{\circ}$ with respect to the radial direction. This ensures that the drift direction is almost perpendicular to the radial direction when the electric field is $1350 \mathrm{~V} / \mathrm{cm}$ and the magnetic field is $1.5 \mathrm{~T}$ in the gas mixture consisting of $49.6 \%$ of argon, $49.6 \%$ of ethane, and $0.8 \%$ ethanol. Three additional wires are located strategically to keep the drift field inside the cell uniform. When a charged particle deposits ionisation in the cell, the maximum drift distance of the ions for both axial and stereo superlayers is less than $40 \mathrm{~mm}$ corresponding to a maximum drift time of $\sim 800 \mathrm{~ns}$. The $r$ resolution is $\lesssim 0.2 \mathrm{~mm}$ per wire while the $z$ resolution is less than $4 \mathrm{~mm}$. The transverse momentum resolution is $\delta p_{T} / p_{T} \sim 0.001 p_{T}$, where $p_{T}$ is measured in units of $\mathrm{GeV} / c$.

The Central Drift Tube (CDT) array is located outside of the CTC and consists of an array of drift tubes operating in the limited streamer mode. The CDT provides $r$ - $\phi$ information but is not used in the analysis described in this thesis.

\subsection{The Calorimeters}

The CDF calorimeter system consists of several electromagnetic and hadronic sampling calorimeters covering $|\eta|<4.2$ with electromagnetic calorimeters placed in front of the hadronic calorimeters relative to the nominal $p \bar{p}$ collision point. The calorimeters are segmented into $\eta$ - $\phi$ projective "towers", each pointing back to the nominal $p \bar{p}$ collision point. The central region $(|\eta|<1.1)$ of the detector is covered by the calorimeters with scintillator active layers; the Central Electromagnetic (CEM) calorimeter, the Central Hadronic (CHA) calorimeter, and the Endwall Hadronic (WHA) calorimeter. The higher $|\eta|$ regions are covered by gas chamber active layer calorimeters; the Plug Electromagnetic (PEM) calorimeter, the Plug 


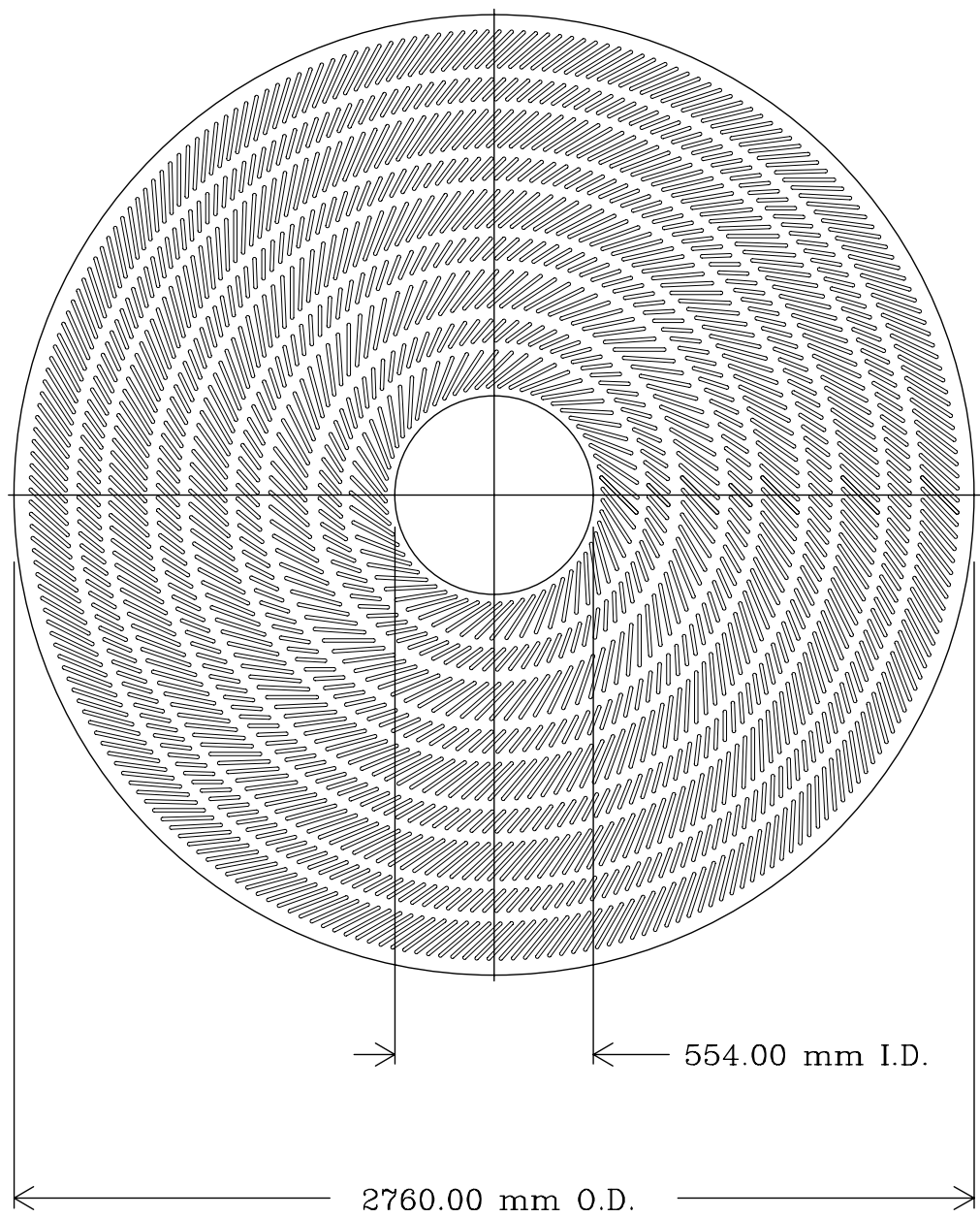

Figure 2.5: CTC endplate viewed from the beam direction. The slots shown are the wire mounts. 


\begin{tabular}{|c|c|c|c|c|c|}
\hline Calorimeter & $|\eta|$ coverage & active & absorber & Thickness & Energy Resolution \\
\hline \hline CEM & $0-1.1$ & scintillator & $\mathrm{Pb}$ & $18 X_{0}$ & $13.7 \% / \sqrt{E} \oplus 2 \%$ \\
\hline $\mathrm{PEM}$ & $1.1-2.4$ & gas chamber & $\mathrm{Pb}$ & $18-21 X_{0}$ & $22 \% / \sqrt{E} \oplus 2 \%$ \\
\hline $\mathrm{FEM}$ & $2.2-4.2$ & gas chamber & $\mathrm{Pb} / \mathrm{Sb}$ & $18 X_{0}$ & $26 \% / \sqrt{E} \oplus 2 \%$ \\
\hline $\mathrm{CHA}$ & $0-0.8$ & scintillator & $\mathrm{Fe}$ & $4.7 \lambda_{0}$ & $50 \% / \sqrt{E} \oplus 3 \%$ \\
\hline WHA & $0.7-1.3$ & scintillator & $\mathrm{Fe}$ & $4.5 \lambda_{0}$ & $75 \% / \sqrt{E} \oplus 4 \%$ \\
\hline $\mathrm{PHA}$ & $1.3-2.4$ & gas chamber & $\mathrm{Fe}$ & $5.7 \lambda_{0}$ & $106 \% / \sqrt{E} \oplus 6 \%$ \\
\hline FHA & $2.4-4.2$ & gas chamber & $\mathrm{Fe}$ & $7.7 \lambda_{0}$ & $137 \% / \sqrt{E} \oplus 3 \%$ \\
\hline
\end{tabular}

Table 2.1: Summary of coverage and energy resolution of the CDF calorimeters. The thickness is given in radiation lengths $\left(X_{0}\right)$ for the electromagnetic calorimeters and absorption lengths $\left(\lambda_{0}\right)$ for the hadronic calorimeters. The energy in the resolution formulae is in units of $\mathrm{GeV}$.

Hadronic (PHA) calorimeter, the Forward/backward Electromagnetic (FEM), and the Forward/backward Hadronic (FHA) calorimeter. The features of these calorimeters are summarized in Table 2.1.

The CEM [33] covers $|\eta|<1.1$ and consists of two arches, with 24 modules in each arch with each module subtending in $15^{\circ}$ in $\phi$. Each module is further segmented into 10 projective towers with equal $\eta$ sizes. A projective tower consists of 21 to 31 layers of $5.0 \mathrm{~mm}$ thick polystyrene scintillator and 20 to 30 layers of $3.2 \mathrm{~mm}$ thick lead absorber depending on the $\eta$ of the tower. The varying number of scintillator-absorber layers is to present nearly constant radiation length thickness for particles with different $\eta$. The scintillator signal from each tower is read out by two photomultiplier tubes. An array of multi-wire proportional chambers is inserted approximately at the shower maximum depth in the CEM to provide measurement of the lateral profile of the electromagnetic shower. The information is used to distinguish between electron and hadron showers.

The CHA [34] covers the region $|\eta|<0.8$ and is located behind the CEM with segmentation identical to the CEM in $\eta$ and $\phi$. The CHA consists of 48 modules, with each module comprising 32 alternating layers of $2.5 \mathrm{~cm}$ thick steel absorber and $1.0 \mathrm{~cm}$ thick acrylic scintillator. Each tower is read out by two photomultiplier tubes.

The WHA [34] is located at each end of the central calorimeter system (CEM and CHA) and covers the region $0.7<|\eta|<1.3$. The WHA consists of 15 alternating 
layers of $5.0 \mathrm{~cm}$ thick steel absorber and $1.0 \mathrm{~cm}$ thick acrylic scintillator. Unlike the CHA, the sampling layers are laid perpendicular to the beam line.

The PEM [35] is a disk-shaped calorimeter $2.8 \mathrm{~m}$ in diameter and $50 \mathrm{~cm}$ thick located at each end of the CTC and covers $1.1<|\eta|<2.4$. The PEM consists of 34 sampling layers of a $2.7 \mathrm{~mm}$ thick lead sheet and wire chambers divided into four quadrants of $\Delta \phi=90^{\circ}$ each. A wire chamber is made of a conductive plastic tube of cross section $7 \mathrm{~mm} \times 7 \mathrm{~mm}$ with $0.8 \mathrm{~mm}$ thick walls and a $50 \mu \mathrm{m}$ diameter gold-plated tungsten anode wire strung in the center. The drift tube wires in each quadrant in a single layer are ganged up and provide a measurement of the longitudinal shower development. Each wire chamber layer is attached to a cathode pad plate segmented into pads of $\Delta \eta \leq 0.09$ and $\Delta \phi=5^{\circ}$. Pads are ganged together longitudinally to form projective towers and used to measure the energy of a shower. The shower position is measured with strips in $\eta$ and $\phi$ directions embedded in layers 6 to 15 .

The FEM [36] is located approximately $6.5 \mathrm{~m}$ from the nominal $p \bar{p}$ collision point and covers $2.2<|\eta|<4.2$. The FEM consists of four quadrants in $\phi$. Each quadrant module has 30 sampling layers, each consisting of a $0.48 \mathrm{~cm}$ thick lead sheet and a chamber of gas proportional tubes with cathode pads. The lead sheets contain $6 \%$ of antimony for rigidity. Each chamber consists of 124 proportional tubes of cross section $0.7 \mathrm{~cm} \times 1.0 \mathrm{~cm}$ formed by an aluminium plate attached with "T" shaped extruded aluminium channels and a cathode plate and $50 \mu \mathrm{m}$ diameter gold-plated tungsten wire strung in each tube. The cathode plate is segmented into pads of $\Delta \eta=0.1$ and $\Delta \phi=5^{\circ}$. Pads are ganged together longitudinally to form projective towers.

The PHA is located behind the PEM covering $1.3<|\eta|<2.4$. The PHA consists of 20 sampling layers of $5.1 \mathrm{~cm}$ thick steel plate and proportional chambers of cross section $14 \mathrm{~mm} \times 8 \mathrm{~mm}$ with cathode pad readout. The cathode pads are segmented into projective towers with size $\Delta \eta=0.09$ and $\Delta \phi=5^{\circ}$.

The FHA [37] covers $2.2<|\eta|<4.2$ with full azimuthal coverage and is located behind the FEM. The FHA is similar to the FEM in construction. Each quadrant consists of 27 layers of a $5 \mathrm{~cm}$ thick steel plate and a chamber of gas proportional tubes with cathode pads. The cathode pads are segmented into $\Delta \eta=0.1$ and $\Delta \phi=5^{\circ}$ projective towers. 


\subsection{The Muon Chambers}

This analysis relies on the ability of the detector to identify energetic muons from the decay of $W$ and $W^{\prime}$ bosons and measure their trajectories. The muons penetrate matter to a much greater degree due to the fact that they are heavy and interact only through the electroweak force. This penetrating property of muons is used to identify them by placing the charged particle detectors beyond intervening material. This material, which is primarily absorbers in the calorimeters in the CDF detector, absorbs a large fraction $(>99 \%)$ of hadrons and electrons before they reach a set of muon chambers. There are three muon chamber subsystems used in this analysis; the Central Muon (CMU) drift chambers, the Central Muon Upgrade (CMP) chambers, and the Central Muon Extension (CMX) chambers [38]. The $\eta-\phi$ coverage of these chambers is shown in Figure 2.6. There are muon chambers in the forward region behind the FHA but they are not used in this analysis.

\subsubsection{The Central Muon Chambers}

The CMU is located outside the CHA at a radial distance of $347 \mathrm{~cm}$ from the beam line and covers $|\eta|<0.7$ with a gap of $3^{\circ}$ in polar angle at $\eta=0$. Figure 2.7 shows the location of the CMU with respect to a central calorimeter wedge. The CMU is segmented in $\phi$ into 24 wedges that lie on the top of each central calorimeter wedge. Each wedge spans $12.6^{\circ}$ in $\phi$, leaving a $2.4^{\circ}$ gap in the muon coverage between wedges. A CMU wedge comprises three modules each spanning $4.2^{\circ}$ in $\phi$. A module is made of four layers of four rectangular drift cells, as shown in Figure 2.8. Overall dimensions of the drift cell are $63.5 \mathrm{~mm}($ width) $\times 26.8 \mathrm{~mm}$ (height) $\times 2261 \mathrm{~mm}$ (length). A

$50 \mu \mathrm{m}$ diameter stainless steel sense wire is located at the center of the cell along the $z$ direction.

Two of the four sense wires in the alternating layers are aligned along the same ray from the $p \bar{p}$ interaction point and the other two sense wires are offset by $2 \mathrm{~mm}$ from the first at the mid point of the chamber, as shown in Figure 2.8. This resolves the ambiguity as to which side of the sense wires a track passes by determining which two sense wires were hit first. The radial arrangement of the sense wires also 


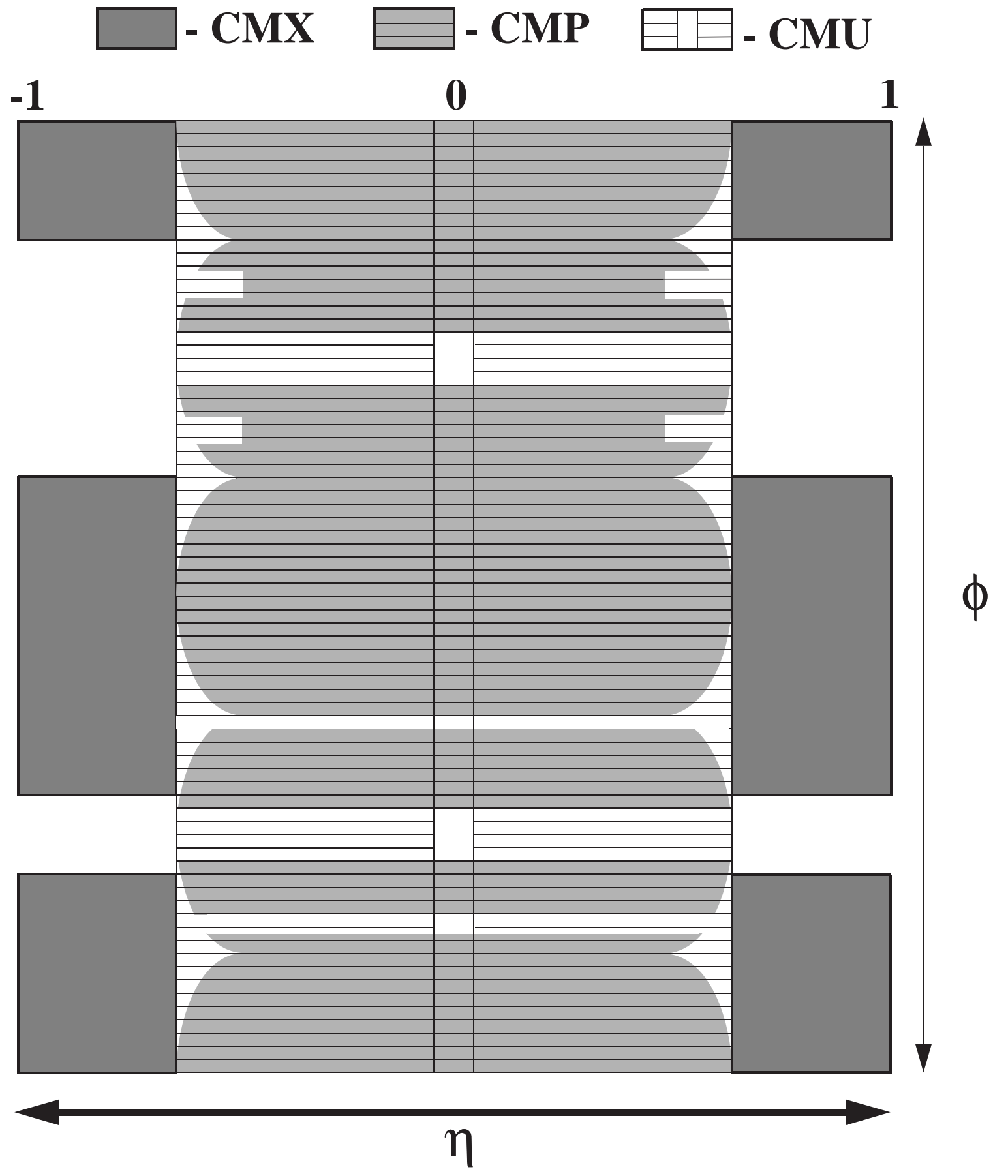

Figure 2.6: Schematic of angular coverage by central muon chambers in $\eta-\phi$ space. The azimuthal angle ranges from 0 to $2 \pi$ (bottom to top). 


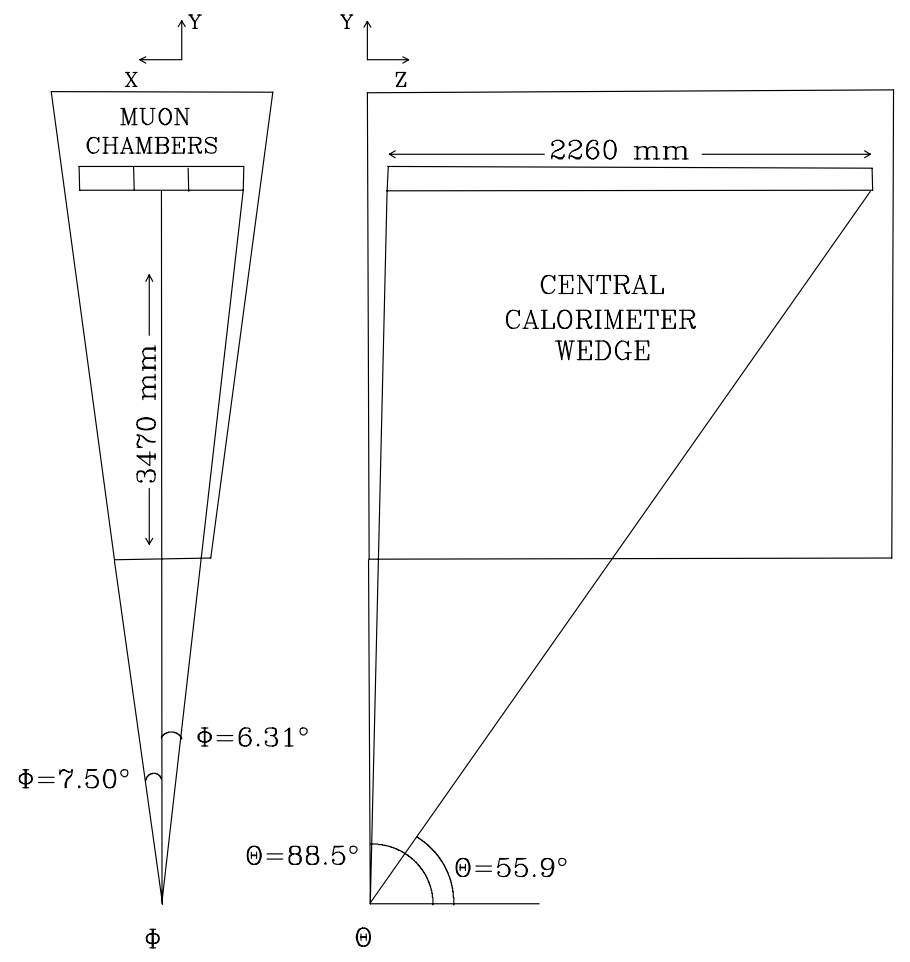

Figure 2.7: Layout of the CMU chamber with respect to a central calorimeter wedge and the $p \bar{p}$ collision point in $\phi$ (left) and in $\theta$ (right).

provides independent of the CTC a crude measurement of the transverse momentum of a particle by relating the angle between the track and the radial direction (see Section 2.6.1).

The muon chambers are operated in the limited streamer mode with a $50 \%$ argon and $50 \%$ ethane gas admixture. The sense wires are normally held at $+3150 \mathrm{~V}$, the top and bottom walls at ground, and the sidewalls at $-2500 \mathrm{~V}$.

The track position along the $z$ direction can be measured by using the "charge division" technique ${ }^{2}$ with a resolution of $1.2 \mathrm{~mm}$. However $z$ position information of the track is not used in this analysis. The position resolution in the $\phi$ direction is $250 \mu \mathrm{m}$.

\footnotetext{
${ }^{2}$ The charge division technique determines the position of a passing particle by measuring the amount of charge reaching the two ends of a resistive wire.
} 


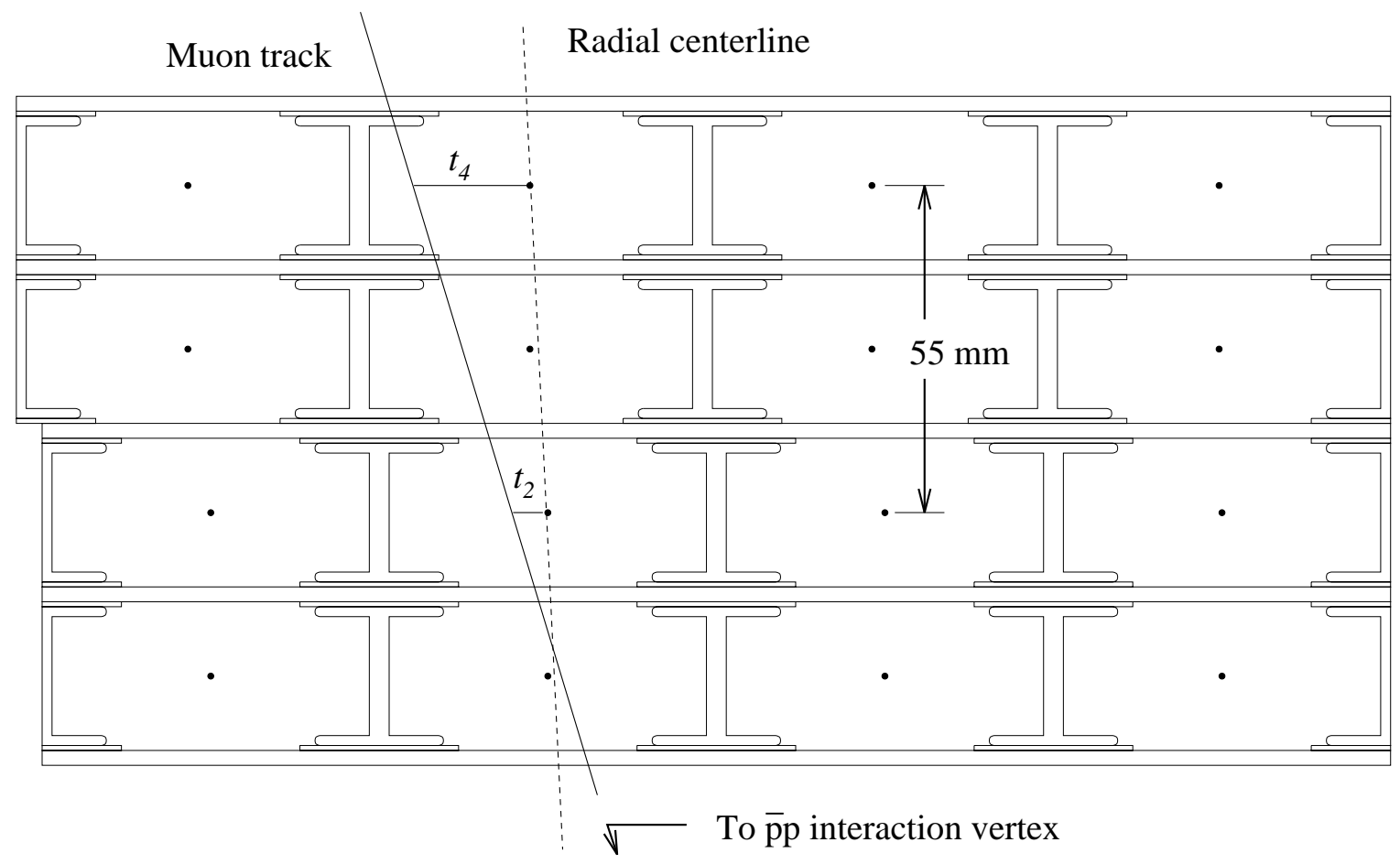

Figure 2.8: Cross section of a single CMU module. Here $t_{2}$ and $t_{4}$ represent the drift times of the electrons for the second and fourth layers in the same module. The difference $t_{4}-t_{2}$ provides the angle between the radial direction and the muon track, thus providing a crude method to determine the transverse momentum of the muon candidate track. 


\subsubsection{The Central Muon Upgrade Chambers}

Since the CMU is located on average 5.4 pion interaction lengths from the $p \bar{p}$ collision point, one in every 220 high energy pions is expected to reach the CMU, leaving little energy in the calorimeters. These "punch-through" hadrons increase the muon trigger rate and pose a serious background to muon identification. The CMP is therefore designed to augment the CMU by requiring muon candidates to pass through additional material between the CMU and a set of additional chambers. The additional material averages 3.0 pion interaction lengths, reducing the the background from hadronic punch-through by a factor of 20 .

The overall shape of the CMP is box-like, using the steel return yoke to provide additional absorption material on the top and the bottom of the CMU and using $60 \mathrm{~cm}$ steel slabs on each side. Because of the box geometry and the limited space available, the CMP covers only part of the CMU acceptance, as is shown in Figure 2.6.

The CMP chambers consist of four half-cell staggered layers of single drift tubes that operate in proportional mode. Tubes are made of $0.26 \mathrm{~cm}$ aluminium extrusions with a $2.5 \mathrm{~cm} \times 15 \mathrm{~cm}$ cross section and are typically $640 \mathrm{~cm}$ long covering $|\eta|<0.6$. A single $50 \mu \mathrm{m}$ diameter gold-plated tungsten wire is strung in the center of the tube and the field shaping cathode strips are laid on the top and the bottom of the tube. The sense wire is held at $+5600 \mathrm{~V}$ and the cathode strips in the center are held at $+3000 \mathrm{~V}$. The outer cathodes strips are held at successively decreasing voltages from the center of the cell. The argon-ethane gas mixture used in the CMU is also used for the CMP chambers.

\subsubsection{The Central Muon Extension Chambers}

The CMX consists of 32 fan-shaped modules (16 modules at each end) with eight layers of drift tubes, covering the region $0.65<|\eta|<1.0$. The modules are arranged to form a conical surface tapered towards the higher $|\eta|$ region with an angle of $45^{\circ}$ with respect to the beam line. The azimuthal coverage of the CMX has a $30^{\circ}$ gap at the top of the detector for the Tevatron Main Ring and the solenoid refrigerator, and a $90^{\circ}$ gap at the bottom of the detector where the conical sections are interrupted by 
the floor. For the CMX, muon candidates have to pass through the calorimeters and the magnet yoke, representing $6.5 \sim 7.5$ interaction lengths.

The drift tubes for the CMX are similar to those for the CMP except that they are $180 \mathrm{~cm}$ long. There are 48 tubes in a CMX module grouped into four logical layers each containing two successive half-cell staggered physical layers. Each physical layer contains six drift tubes. Figure 2.9 illustrates of the drift tube layout in a CMX module. This half-cell staggered configuration allows the average muons to traverse six drift tubes.

Because the maximum drift time of ionisation in the CMX chambers is long $(\sim$ $1.4 \mu \mathrm{s})$ compared to the spread of arrival times of the background particles produced by the Main Ring and particles scattering from the beam line, a scintillator array mounted on each side of the CMX module is used to provide background rejection and fast triggering. There are four scintillators per CMX module, providing a high degree of segmentation. A muon candidate is required to generate a coincidence between an inner and an outer scintillator adjacent to a track segment in the CMX drift chambers.

\subsection{The Trigger Systems}

The proton and antiproton beam bunch crossings in the Tevatron occurred every $3.5 \mu \mathrm{s}$, corresponding to a rate of $286 \mathrm{kHz}$ at the $\mathrm{B} \varnothing$ interaction point. With a typical instantaneous luminosity of $\mathcal{L}=10^{31} \mathrm{~cm}^{-2} s^{-1}$ and the measured total $p \bar{p}$ cross section of $(80.03 \pm 2.24) \times 10^{-27} \mathrm{~cm}^{2}$ at $\sqrt{s}=1.8 \mathrm{TeV}$, on average $\sim 1.6 p \bar{p}$ interactions per bunch crossing was expected [28].

An interaction recorded by the CDF detector generated $\sim 165 \mathrm{kB}$ of digitised information. The offline data handling capacity of the experiment limited this rate of recorded interactions to be $\sim 8 \mathrm{~Hz}$. These necessitated a trigger system that reduced the recorded event rate by selecting interesting physics events with as little deadtime ${ }^{3}$ as possible.

\footnotetext{
${ }^{3}$ Deadtime is the fraction of time where incoming data cannot be considered because the trigger system or detector has not completed processing of an earlier interaction.
} 

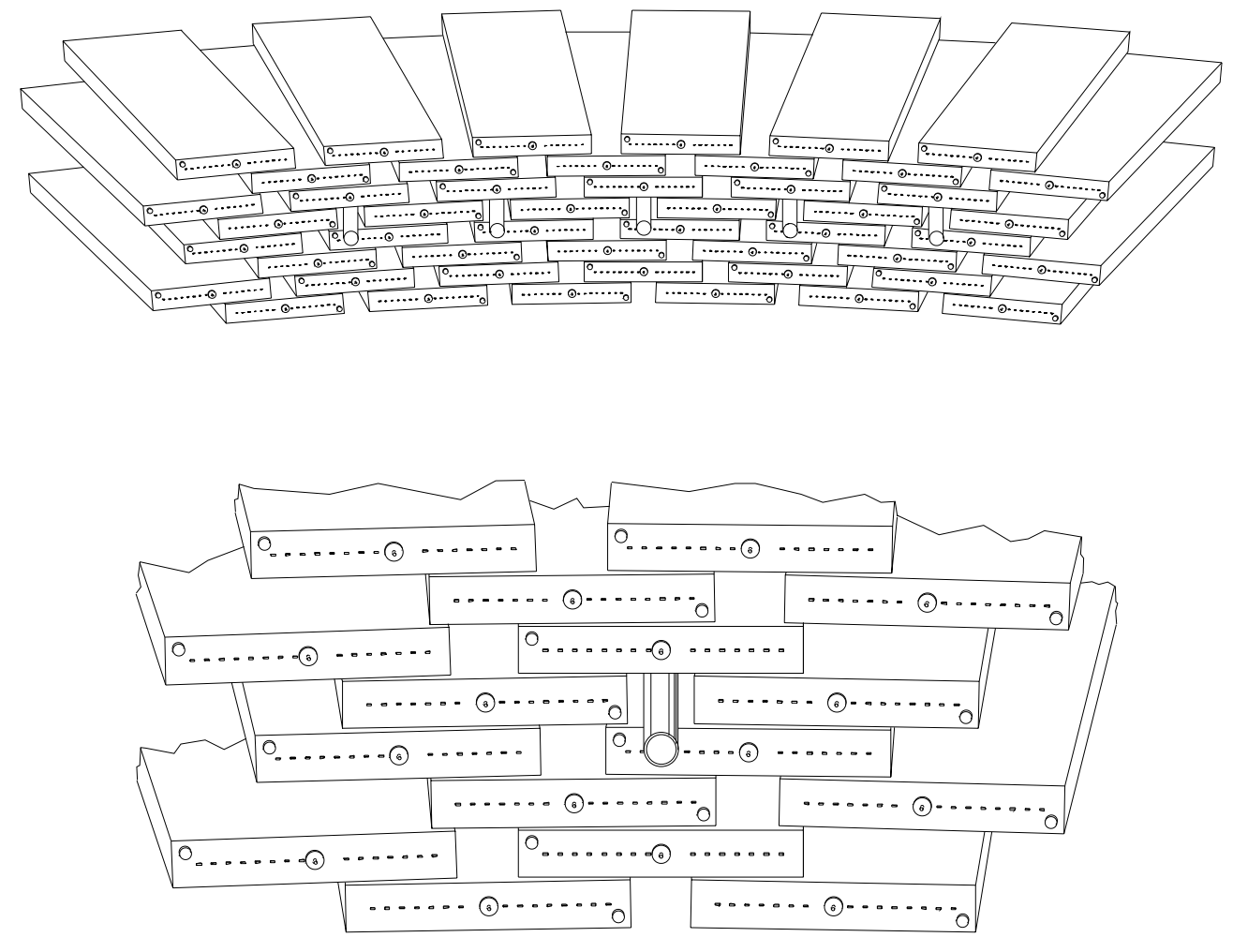

Figure 2.9: Drift tube layout for a $15^{\circ}$ central muon extension (CMX) module (top). Also shown is the close up view of the staggered cell configuration (bottom). The rods in the diagrams are used to attach the module to the support arch. 
The trigger system employed at CDF had three levels. Each level made decisions using successively more detailed requirements based on more information, thus taking more time to make a decision. All of the triggers were programmable in several respects so that each could be tuned to different run conditions and physics requirements. The decision at each level was based on a logical "OR" of requirements that were designed to select events with different characteristics. The overall goal was to operate the detector with less than $15 \%$ loss in efficiency due to deadtime. The general description of the trigger system is presented here. The trigger requirements specific to this analysis are given in Section 3.2 .

\subsubsection{Level 1}

The Level 1 trigger decision to pass an event to the next level must be made within $3.5 \mu \mathrm{s}$ in order to ensure the detector could consider the next bunch crossing. To accommodate this requirement, the analog signals from selected detector subsystems were read and processed with dedicated FASTBUS-based electronics. By the time the Level 1 decision was made, the trigger system had accumulated information concerning the global features of the event. The Level 1 decision was based solely on information from the calorimeters and the muon chambers.

The Level 1 calorimeter trigger decision was made from the analog signal coming from either the scintillator phototubes in the central calorimeters or the cathode pads in the gas calorimeters. To reduce the number of signals, the calorimeters were segmented into logical trigger towers of 0.2 in $\eta$ and $15^{\circ}$ in $\phi$. The energy from trigger towers that were above thresholds were weighted in $\theta$ to determine transverse energy, $E_{T} \equiv E \sin \theta$, and then summed over separately for different calorimeters. If the total $E_{T}$ determined in this manner exceeded a preset threshold, the event was accepted by the Level 1 trigger.

The muon component of the Level 1 trigger decision was based on the transverse momentum, $p_{T} \equiv p \sin \theta$, of a muon track candidate observed in the muon chambers. The time difference between the signals arriving from pairs of sense wires aligned 
in the radial direction ${ }^{4}$ measured the angle between the ray originating from the $p \bar{p}$ interaction point and a muon track candidate (see Figure 2.8). Due to the axial magnetic field inside the solenoid, this angle is a measure of the curvature of the muon candidate trajectory and is inversely proportional to the $p_{T}$ of the track. The muon triggers required the difference between the arrival time of the signals from a sense wire pair in any of the alternative layers in a muon chamber to be less than a preset value, corresponding to the requirement of a specific minimum $p_{T}$ requirement.

\subsubsection{Level 2}

Upon the Level 1 trigger accept, the analogue event information in the CDF detector was held for up to $20 \mu \mathrm{s}$. During this time, the Level 2 trigger system reconstructed the event information and created physics objects such as electrons, muons, and jet candidates and then made trigger decisions based on the criteria imposed on the physics objects.

At Level 2, a fast track reconstruction was performed with the Central Fast Tracker (CFT), a hardware track finder. The CFT used timing information from five axial superlayers of the CTC (see Section 2.3) to determine the $p_{T}$ and $\phi$ of charged particle tracks. Two types of timing information were used; prompt hits and delayed hits. Prompt hits were the signals from the sense wires within $80 \mathrm{~ns}$ from the beam bunch crossing time. The delayed hits occurred $500 \sim 650$ ns after the beam bunch crossing with the same time interval as the prompt hits. By comparing the hit patterns through a look-up table, the $p_{T}$ of individual tracks could be measured with a resolution of $\delta p_{T} / p_{T} \sim 0.035 p_{T}$, where $p_{T}$ is in units of GeV/c. The CFT generated a list of track candidates in eight $p_{T}$ bins and this information was passed to the rest of the Level 2 trigger system to be used to match the track candidates with track stubs in the muon chambers and calorimeter clusters.

The formation of calorimeter energy clusters was also performed in Level 2. Two sets of thresholds, the "seed" and "shoulder", were applied on trigger towers, where the seed threshold was higher than the shoulder threshold. When a trigger tower

\footnotetext{
${ }^{4}$ This is not true in a strict sense. As described in Section 2.5.1, a pair of sense wires are aligned radially and the other pair is shifted by some distance.
} 
passed the seed threshold, it formed the basis for a cluster and the four adjacent trigger towers were checked to see if any of them passed the shoulder threshold. A trigger tower was added to the cluster if it passed the shoulder threshold. Additional neighbouring towers were added to the cluster if they passed the shoulder threshold and did not belong to other clusters. Electromagnetic energy clusters and total energy clusters were formed in a two pass process, with the former being used in electron triggers and the latter being used in jet triggers. The trigger towers containing clusters were matched to the CFT track list in $\phi$. After the clustering was performed, the $E_{T}$, $\phi$, and $\eta$ of each cluster were calculated. Global sums of calorimeter energy such as total energy, total transverse energy, total missing transverse energy, and total cluster energy were also calculated.

The hits in the muon chambers were matched to the CFT track list. The CFT tracks were extrapolated to the muon chambers and a match was declared if the extrapolated track and the hits in the muon chambers were within a certain window in $\phi, 15^{\circ}$ in the beginning of the first data taking period and $5^{\circ}$ during the remaining data taking period. A muon candidate was required to have a matching CFT track with $p_{T}$ greater than a given threshold. The typical threshold in this analysis was 9.2 and $12 \mathrm{GeV} / c$, depending on the running period.

\subsubsection{Level 3}

When an event was accepted by at least one Level 2 trigger, all channels in the CDF detector were digitised and read out by the Data Acquisition (DAQ) system (see Section 2.7) and sent to the Level 3 processor farm that consisted of a number of commercial processors. ${ }^{5}$ There were 48 processors in the Level 3 processor farm during Run 1A and 64 processors in Run 1B. A single processor was assigned to each event passing the Level 2 trigger, and the event data was transferred to the processor using a sophisticated high-speed network, known as Ultranet. This system read the data from the FASTBUS electronics cards using a $\mathrm{VME}^{6}$ based microprocessor that then transferred the data via the Ultranet hub to a processor in Level 3. Up to 48

\footnotetext{
${ }^{5}$ Manufactured by Silicon Graphics Inc., running under the IRIX operating system.

${ }^{6}$ VME stands for "Versa Module Eurocard", a crate-based electronics system.
} 
events in Run 1A and 64 events in Run 1B could be processed in parallel, freeing up the detector, the Level 1, and the Level 2 trigger systems for the subsequent beam bunch crossings.

An event was reconstructed in Level 3 using a special version of the full event reconstruction programme that was mostly written in the FORTRAN programming language. The trigger decision was based on combinations of physics objects reconstructed by detailed algorithms. There were numerous trigger streams that fired on different physics requirements. The triggers relevant to the analysis in this thesis required muon candidates to deposit a certain amount of energy in the hadronic calorimeter, match with a CTC track extrapolated to the muon chambers, and to have a minimum transverse momentum. When any of the Level 3 trigger requirements were satisfied, the event information was written to the data storage medium.

\subsection{The Data Acquisition System}

The CDF DAQ and trigger system had a hierarchical structure, as shown in Figure 2.10. At the lowest level of this structure, analog signals from various detector components were amplified, discriminated, and reshaped. A small fraction of analog signals that contained the rudimentary event information was sent to the Level 1 and Level 2 trigger system through dedicated cables from the front end electronic cards. When an event was accepted by a Level 1 and a Level 2 trigger, an accept signal was sent to the trigger supervisor, a FASTBUS module that coordinated the readout of the entire event. The trigger supervisor signalled the front end electronics cards to digitise the data for the event and instructed FASTBUS Readout Controllers (FRCs) to read digitised signals from the front end electronics cards. The FRCs sent this data to Scanner CPUs, or SCPUs, over a 16-bit parallel bus. The SCPUs, VME modules with Motorola 68030 processors running the VxWorks real-time operating system, moved the event data to a Level 3 processor through the Ultranet hub, a commercial data switch. The scanner manager, a VME module with a Motorola 68030 processor, used Scramnet, a reflective memory network, to instruct the SCPUs to send the event data to an unoccupied Level 3 processor (see Section 2.6.3). The trigger super- 
visor interface allowed communication between the trigger supervisor and the scanner manager.

After an event was accepted by a Level 3 trigger, it was sent to the consumer server, a process running on a dedicated two-processor Challenge , $^{7}$ via the Ultranet hub. The consumer server wrote the data on local disk, and the data were subsequently written on to $8 \mathrm{~mm}$ tapes. The event data could be accessed by various "consumer" processes over ethernet. The consumers were diagnostic processes that monitored trigger rate, luminosity, detector performance, and a select set of physics processes. The data taking was initiated and terminated by an operator issuing commands to run control, a process running on a VAX computer. ${ }^{8}$ The commands and messages between run control and the scanner manager were relayed by the user control interface, a VME module with a Motorola 68030 processor running the VxWorks real-time operating system.

\footnotetext{
${ }^{7}$ Manufactured by Silicon Graphics, Inc.

${ }^{8}$ Manufactured by Digital Equipment Corporation.
} 


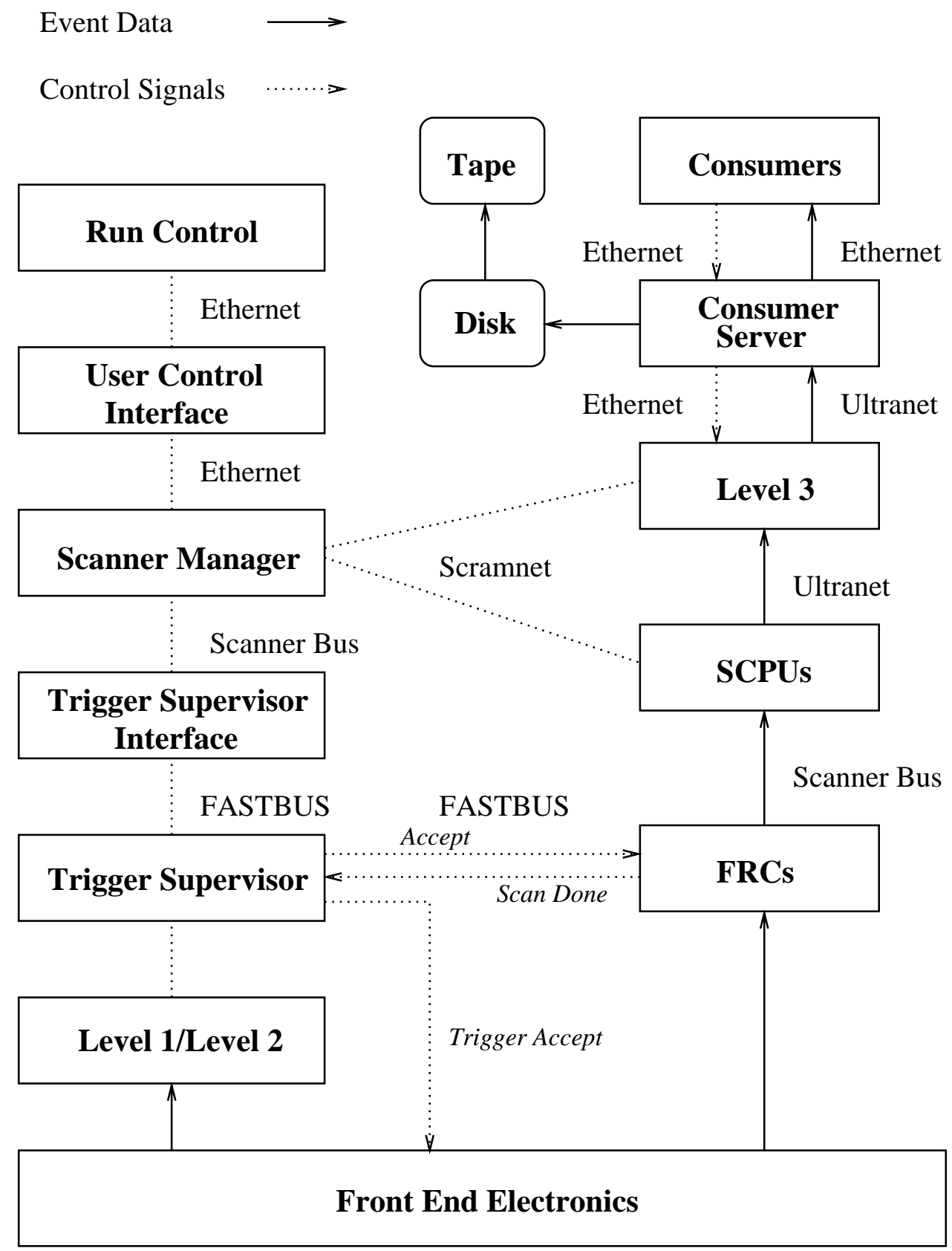

Figure 2.10: A schematic of the CDF data acquisition and trigger system. The individual components are described in the text. 


\section{Chapter 3}

\section{Event Selection}

In this chapter, the data sets used in this analysis are defined, the experimental signature of the $W^{\prime} \rightarrow \mu \nu$ process is described, and specific requirements imposed on the data to identify the candidate events and to reject the backgrounds are outlined. Characteristics of the $W / W^{\prime} \rightarrow \mu \nu$ candidate sample, such as the transverse mass distribution of the candidates, are discussed.

\subsection{The Data Sample}

The data samples used in this study were recorded with the CDF detector in the period from 1992 to 1995. During that time, the Tevatron collided protons and antiprotons at $\sqrt{s}=1.8 \mathrm{TeV}$. There were two "runs" during this period. The first run, referred to as Run 1A, began in August 1992 and ended in May 1993 with a recorded integrated luminosity of $\int \mathcal{L} d t=19 \pm 1 \mathrm{pb}^{-1}$. After the end of Run $1 \mathrm{~A}$, several upgrades were made to the CDF detector: The replacement of SVX with SVX' (see Section 2.3) and the upgrade of the DAQ system (see Section 2.7) took place. The second run, referred to as Run 1B, began in January 1994 and ended in July 1995 with a recorded integrated luminosity of $\int \mathcal{L} d t=88 \pm 7 \mathrm{pb}^{-1}$.

During the Run 1B data taking period, improvements in the beam optics of the Tevatron allowed for higher instantaneous luminosity than achieved during Run 1A. The mean instantaneous luminosity increased from $\mathcal{L} \sim 3.6 \times 10^{30} \mathrm{~cm}^{-2} \mathrm{~s}^{-1}$ for Run $1 \mathrm{~A}$ to $\mathcal{L} \sim 7.9 \times 10^{30} \mathrm{~cm}^{-2} \mathrm{~s}^{-1}$ for Run $1 \mathrm{~B}$. The peak instantaneous luminosity in 
Run $1 \mathrm{~B}$ was $\mathcal{L} \sim 2.5 \times 10^{31} \mathrm{~cm}^{-2} \mathrm{~s}^{-1}$. This increase in luminosity required certain triggers to be prescaled (i.e. only a fraction of the triggers were recorded), broadened underlying event distributions (see Section 5.2.1), and degraded the track momentum resolution (see Section 5.2.2).

The analysis presented here used both Run $1 \mathrm{~A}$ and Run $1 \mathrm{~B}$ data sets. When necessary, the differences in the run conditions for the data sets were accounted for so that the data sets could be treated as one data set, which will be referred to as Run 1A+1B, henceforth.

\subsection{Triggers and Production}

The events for this analysis were first selected by the three level trigger system, as described in Section 2.6. The Level 1 muon trigger required a muon stub, which was defined by at least two hits in a set of muon chambers (see Section 2.6.1), with $p_{T}>6 \mathrm{GeV} / c$ in either the CMU or the CMP, or with $p_{T}>10 \mathrm{GeV} / c$ in the CMX. The Level 1 CMX trigger was not available for most of Run $1 \mathrm{~A}$ and, therefore, was not used in this analysis for selecting events during Run $1 \mathrm{~A}$.

The Level 2 trigger extrapolated CFT tracks to the muon chambers and matched them to muon stubs that passed the Level 1 requirements. In Run $1 \mathrm{~A}$, the CFT track of a muon candidate was required to have $p_{T}>9.2 \mathrm{GeV} / c$ and its extrapolation to the muon chambers to be within $15^{\circ}\left(5^{\circ}\right)$ of a muon stub in azimuth in the early (late) part of Run 1A. There were three triggers used to select events for this analysis: CMU_CMP_CFT_9_2, CMUP_CFT_9_2_5DEG, and CMUNP_CFT_9_2_5DEG. The CMU_CMP_CFT_9_2 trigger required a muon candidate to have a matching muon stub in the CMU in the region where there was no CMP coverage or to have matching muon stubs in both the CMU and CMP where the region was covered by both (see Figure 2.6). The CMU_CMP_CFT_9_2 trigger was gradually replaced by the CMUP_ CFT_9_2_5DEG trigger and the CMUNP_CFT_9_2_5DEG trigger in the course of Run 1A. The CMUP_CFT_9_2_5DEG trigger required a muon candidate to have muon stubs in both the CMU and CMP, and the CMUNP_CFT_9_2_5DEG trigger required a muon candidate to have a muon stub in the CMU in the region where there is no CMP coverage. 
In Run 1B, a muon candidate was required to have a CFT track with $p_{T}>$ $12 \mathrm{GeV} / c$ and a matching muon stub within $5^{\circ}$ in azimuth that passed the Level 1 requirements. There were three triggers used in this analysis: CMUP_CFT_12_5DEG, CMNP_CFT_12_5DEG, and CMX_CFT_12_5DEG. The CMUP_CFT_12_5DEG trigger required a muon candidate to have muon stubs in both CMU and CMP in the region covered by both the CMU and CMP. The CMNP_CFT_12_5DEG trigger required a muon candidate to have a muon stub in the CMU in the region that is not covered by the CMP. The CMX_CFT_12_5DEG trigger required a muon candidate to have a muon stub in the CMX. Due to the high trigger rates, both CMNP_CFT_12_5DEG and CMX_CFT_12_5DEG triggers were dynamically prescaled, i.e. they were limited to never exceed a certain rate. Approximately $60 \%$ of these triggers were therefore rejected due to this prescaling over the Run 1B data taking period.

In Level 3, a muon candidate was required to have a reconstructed CTC track with $p_{T}>18 \mathrm{GeV} / c$ and a muon stub that was within $5 \mathrm{~cm}\left(\sim 2^{\circ}\right)$ of the track extrapolated to the CMU in azimuth or within $10 \mathrm{~cm}$ to a muon stub either in the CMP or CMX. The energy in the hadronic calorimeter associated with the muon candidate track was required to be less than $6 \mathrm{GeV}$.

These events were subsequently processed completely to apply calibration constants, reconstruct charged particle trajectories and analyse calorimeter energy depositions. There were $8.3 \times 10^{4}$ events and $6.5 \times 10^{5}$ events in Run $1 \mathrm{~A}$ and Run $1 \mathrm{~B}$ samples, respectively. ${ }^{1}$

\section{3 $\quad W^{\prime} \rightarrow \mu \nu$ Event Identification}

In the scope of this search, ${ }^{2}$ the signature of a $W^{\prime} \rightarrow \mu \nu$ decay would be similar to that of a $W \rightarrow \mu \nu$ decay with a muon and a neutrino from a heavier $W^{\prime}$ boson decay typically having higher transverse momenta than those from a $W$ boson decay. Also, due to the phase space available to the partons in the initial state, $W^{\prime}$ bosons are expected to be produced more centrally than $W$ bosons (see Figure 5.1).

\footnotetext{
${ }^{1}$ These samples included events accepted by other Level 2 muon triggers.

${ }^{2}$ The neutrino from a $W^{\prime}$ boson decay is light and does not decay inside the detector volume.
} 
A neutrino is not detected directly by the CDF detector. Its presence, however, is inferred from the observation of a large imbalance in the vector sum of the transverse energy flow in the event, or missing $E_{T}\left(\mathbb{F}_{T}\right)$. However, since the longitudinal momentum of the colliding partons in the lab frame is unknown, the longitudinal momentum of the neutrino cannot be reconstructed. Therefore, the invariant mass of the $\mu \nu$ system cannot be fully reconstructed. Instead, the transverse mass, $M_{T}$, of the $\mu \nu$ system, which is the analogue of the invariant mass in the plane transverse to the beam, is calculated as

$$
M_{T}=\sqrt{\left(p_{T}^{\mu}+\mathbb{H}_{T}\right)^{2}-\left(\vec{p}_{T}^{\mu}+\vec{H}_{T}\right)^{2}}
$$

where $\vec{p}_{T}^{\mu}$ is the transverse momentum of the muon and $\vec{H}_{T}$ represents the transverse momentum of the neutrino.

The transverse mass distribution for a $W$ or $W^{\prime}$ boson peaks just below the actual mass of the boson and quickly falls off above the peak. It also exhibits a Jacobian smearing below the boson mass.

In this analysis, the $W \rightarrow \mu \nu$ candidate events were used to normalise the expected yield of $W^{\prime} \rightarrow \mu \nu$ events. This removes any systematic effects common to both our model of the $W \rightarrow \mu \nu$ and $W^{\prime} \rightarrow \mu \nu$ processes and their detection. The existence of a $W^{\prime} \rightarrow \mu \nu$ process would manifest itself as a "bump" in the transverse mass distribution above the standard model background.

\subsection{Primary Muon Selection}

A muon candidate was identified as an object that has a track in the CTC with a matching muon stub in the muon chambers and a small amount of energy in the calorimeter towers pierced by the track. The selection criteria for the muon candidate with the highest transverse momentum in an event, referred to as the primary muon candidate, can be placed into three groups; the event vertex requirements, the track quality requirements, and the requirements pertaining to the intrinsic characteristics of a muon. 


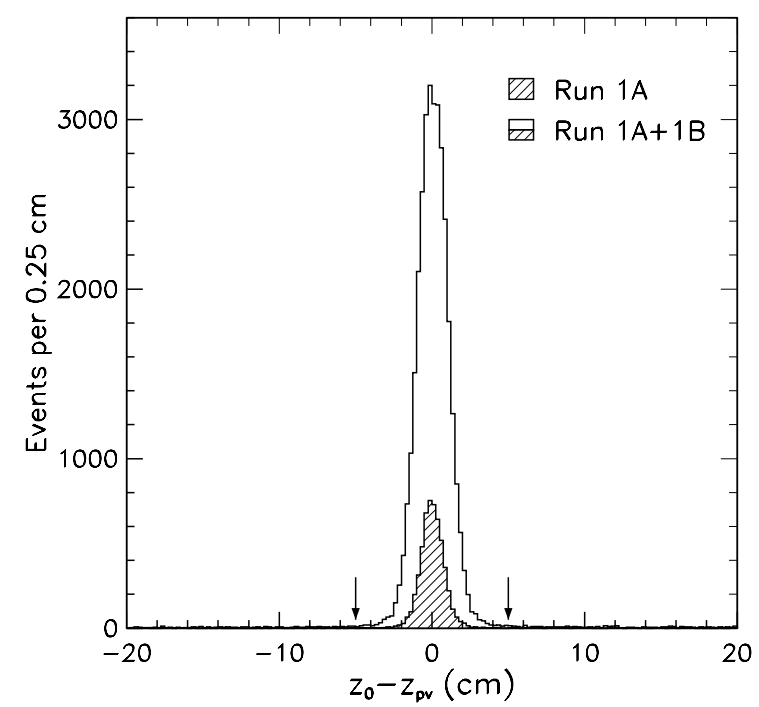

Figure 3.1: The $z_{0}-z_{p v}$ distribution of the primary muon candidates. All the cuts for the $W / W^{\prime} \rightarrow \mu \nu$ event selection were applied except the cut on $z_{0}-z_{p v}$. The arrows indicate where the cuts were made.

A primary muon candidate was required to originate from a $p \bar{p}$ collision point, or a primary vertex. The longitudinal coordinate of the primary vertex, $z_{p v}$, was determined by charged particles observed in the VTX. The "quality" of each vertex candidate depended on the number of tracks in the VTX pointing to the vertex. Because multiple interactions were common (more so in Run 1B than in Run 1A), the high quality vertex ${ }^{3}$ that was closest to the longitudinal coordinate of the primary muon candidate at the point closest to the beam position, $z_{0}$, was identified as the primary vertex. The primary muon candidate was required to have a primary vertex within $5 \mathrm{~cm}$ of its $z_{0}$. The $z_{0}-z_{p v}$ distribution of the $W / W^{\prime} \rightarrow \mu \nu$ event candidates is shown in Figure 3.1.

The transverse coordinates of the primary vertices in an event were calculated from the run-averaged ${ }^{4}$ beam position measured by the SVX. The beam position typically varied less than $10 \mu \mathrm{m}$ over a single run period whereas the beam spot size was on the order of $50 \mu \mathrm{m}$. The impact parameter, $d_{0}$, defined as the closest

\footnotetext{
${ }^{3}$ The vertices were placed into 12 classes with class 12 being the highest quality. The vertices with class 10 or higher were considered as primary vertex candidates in this analysis.

${ }^{4} \mathrm{~A}$ "run", in this context, refers to a period of uninterrupted data collection, usually lasting from several hours to up to 20 hours.
} 

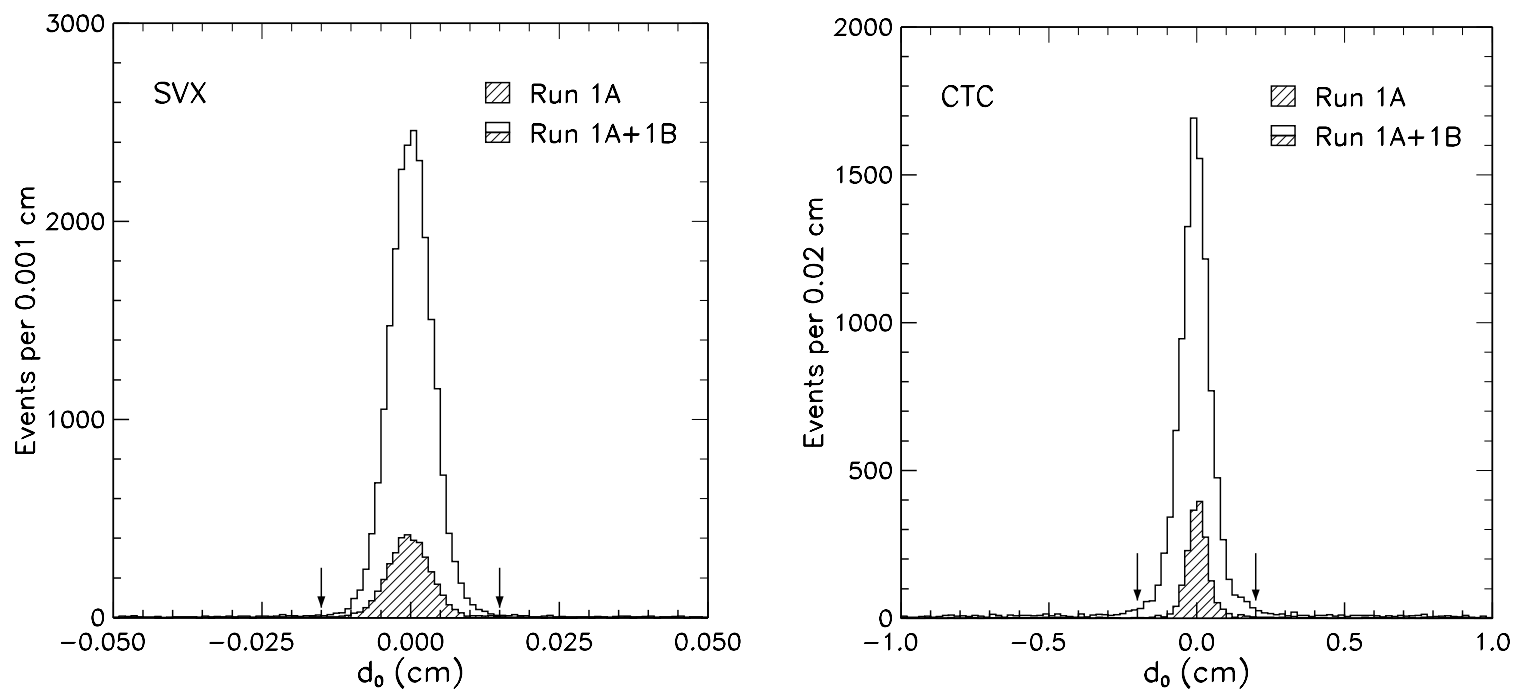

Figure 3.2: The impact parameter distributions of the primary muon candidates measured with the SVX $+\mathrm{CTC}$ (left) and the CTC (right). All the requirements on the $W / W^{\prime} \rightarrow \mu \nu$ event candidates were applied except the cut on $d_{0}$. The arrows indicate where the cuts were made. Note the difference in horizontal scale.

distance between the beam position and the muon track candidate, was required to be less than $0.15 \mathrm{~mm}$ if the track had two or more hits in the SVX, or to be less than $2 \mathrm{~mm}$ otherwise. The impact parameter distributions of the $W / W^{\prime} \rightarrow \mu \nu$ event candidates are shown in Figure 3.2. Primary vertex information was used to provide an additional fit point for fitting a track to hits in the CTC (a beam constrained fit) and to remove tracks that did not originate from an interaction point, such as cosmic rays, and badly reconstructed tracks.

Track quality requirements were imposed to remove poorly measured tracks. The quality of a track candidate depends on the number of hits used in the CTC as well as the distribution of the hits. ${ }^{5}$ To ensure that the trajectory of the primary muon traversed all nine superlayers of $\mathrm{CTC}$, the track was required to intersect one of the CTC endplate planes at a radius, $r_{\text {exit }}$, greater than $132 \mathrm{~cm}$, the radial distance of the outer-most CTC sense wire layer. Also, the CTC track of the primary muon candidate was required to have the number of hits in the CTC superlayers through 3

\footnotetext{
${ }^{5}$ The rudimentary requirement for successful track reconstruction was that there should be at least two axial superlayers with at least four hits each and at least two stereo superlayers with at least two hits each.
} 


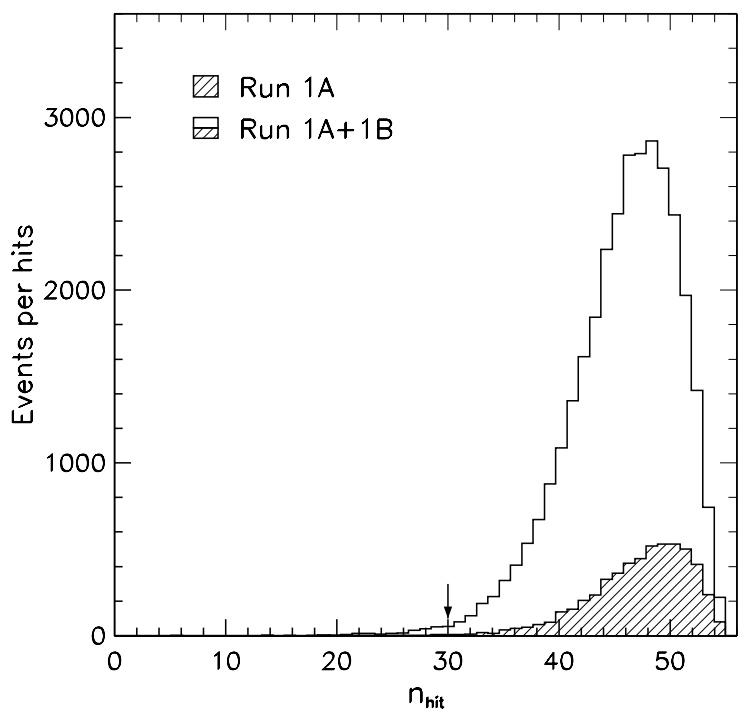

Figure 3.3: The number of hits in the CTC superlayers 3 to $8, n_{\text {hit }}$. All the cuts were applied except the cut on $n_{\text {hit }}$. The arrow indicates where the cut was made.

to $8, n_{\text {hit }}$, to be at least 30 out of possible 54 (see Section 2.3). Figure 3.3 shows the hit distribution for the $W / W^{\prime} \rightarrow \mu \nu$ event candidates. The muon candidates were required to have $z_{0}$ within $60 \mathrm{~cm}$ of the nominal $p \bar{p}$ collision point, $z=0$.

The main background in this muon candidate sample was expected to come from pions whose shower was not been fully contained by the calorimeters. The primary muon candidates were required to have $E_{e m}<2 \mathrm{GeV}$ and $E_{\text {had }}<6 \mathrm{GeV}$, where $E_{e m}$ and $E_{\text {had }}$ were the energy deposited in the electromagnetic calorimeter and the hadronic calorimeter, respectively, in the towers the muon candidate intersected. Even though $E_{\text {had }}<6 \mathrm{GeV}$ was required for muon candidates in Level 3, it was imposed here again to remove those events where a muon candidate other than the primary muon candidate had fired the triggers. The $E_{e m}$ and $E_{\text {had }}$ distributions of the $W / W^{\prime} \rightarrow \mu \nu$ event candidates are shown in Figure 3.4. In order to remove spurious tracks, $E_{\text {had }}+E_{e m}>0.1 \mathrm{GeV}$ was required for the muon candidates.

Pions that are misidentified as muon candidates usually occur within jets and are, on average, not isolated from other energy flow. The isolation variable, Iso, was defined as

$$
I s o \equiv E_{T}(R<0.4)-E_{T}^{\mu} \text {, }
$$



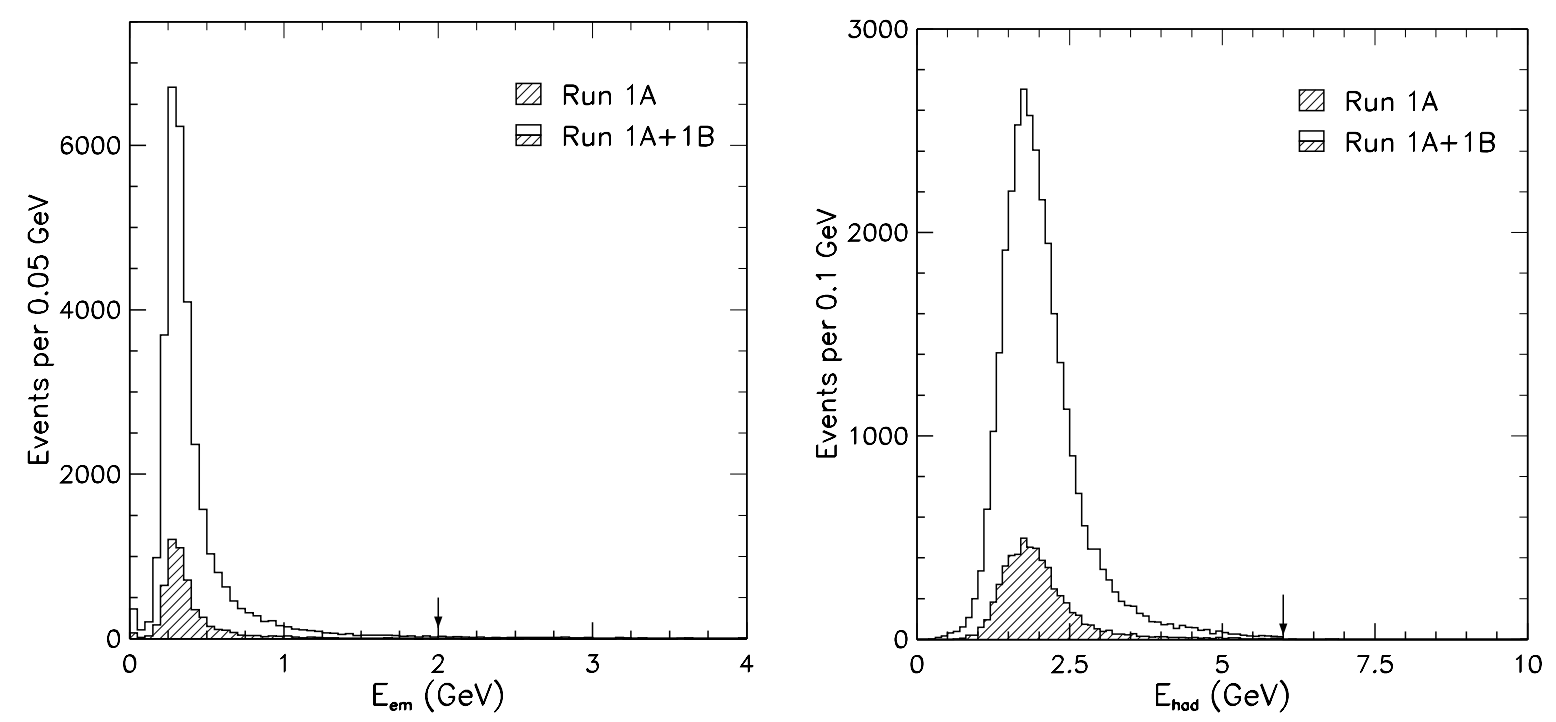

Figure 3.4: The primary muon $E_{e m}$ and $E_{\text {had }}$ distributions. All the cuts were applied except the cut on $E_{e m}$ and $E_{\text {had }}$, respectively. The arrows indicate where the cuts were made.

where $E_{T}(R<0.4)$ was the transverse energy deposited within a cone of $R=0.4$ around the muon candidate, where $R \equiv \sqrt{(\Delta \phi)^{2}+(\Delta \eta)^{2}}$, and $E_{T}^{\mu}$ was the transverse energy deposited by the muon candidate. ${ }^{6}$ The muon candidate was required to have Iso $<3 \mathrm{GeV}$, as shown in Figure 3.5. The matching in azimuth between the CTC track extrapolated to the muon chambers and the muon stubs in the muon chambers was required to satisfy $\left|\Delta x_{C M U}\right|<2 \mathrm{~cm},\left|\Delta x_{C M P}\right|<5 \mathrm{~cm}$ or $\left|\Delta x_{C M X}\right|<5 \mathrm{~cm}$. Finally, the primary muon candidate was required to have $p_{T}>20 \mathrm{GeV} / c$.

\subsection{Missing Transverse Energy}

The missing transverse energy was calculated as

$$
\vec{\not}_{T}=-\sum_{i} \vec{E}_{T_{i}}-\sum_{j}\left(\vec{p}_{T_{j}}^{\mu}-\vec{E}_{T_{j}}^{\mu}\right)
$$

\footnotetext{
${ }^{6}$ From here on, $\eta$ is calculated from $\theta$ measured at the track vertex of the muon candidate, as opposed to $\theta$ measured at the nominal $p \bar{p}$ collision point as defined in Equation 2.1.
} 


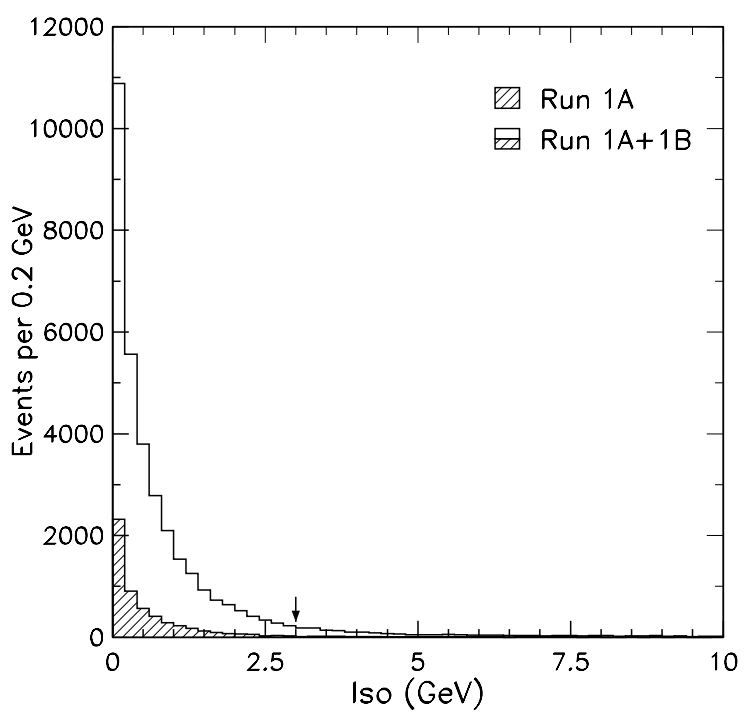

Figure 3.5: The primary muon Iso distributions. All the cuts were applied except the cut on Iso. The arrow indicates where the cut was made.

where $\vec{E}_{T_{i}}$ and $\vec{E}_{T_{j}}^{\mu}$ are the transverse energy of all calorimeter towers in the detector and those towers intersected by the muon candidates, respectively. Because the CDF calorimeter system is not completely hermetic, the particles can go through uninstrumented regions causing the jet energy to be significantly underestimated. Also, the calorimeter response is not linear to the incident particle energy. The vector sum of the transverse energy, $\sum \vec{E}_{T_{i}}$, was corrected by applying corrections for this calorimeter response to each jet energy cluster of a cone of $R=0.7$ with uncorrected $E_{T}$ greater than $5 \mathrm{GeV}$, and then propagating this correction to the $\overrightarrow{\mathbb{F}}_{T}$. The $\overrightarrow{\mathbb{F}}_{T}$ was also corrected for the primary and a secondary muon candidates if it existed. The secondary muon candidate for the $\vec{\not}_{T}$ correction was required to pass $I s o<3 \mathrm{GeV}$, $E_{e m}<2 \mathrm{GeV}, E_{\text {had }}<6 \mathrm{GeV}, E_{\text {had }}+E_{\text {em }}>0.1 \mathrm{GeV}$, and $\Delta x$ matching cuts. Events were required to satisfy $\mathbb{F}_{T}>20 \mathrm{GeV}$ after these corrections.

\section{6 $Z / \gamma \rightarrow \mu \mu$ Rejection}

The $Z / \gamma \rightarrow \mu \mu$ process yields the largest background contribution to the $W / W^{\prime} \rightarrow \mu \nu$ event candidate sample. To remove the evident $Z / \gamma \rightarrow \mu \mu$ events, events were rejected 


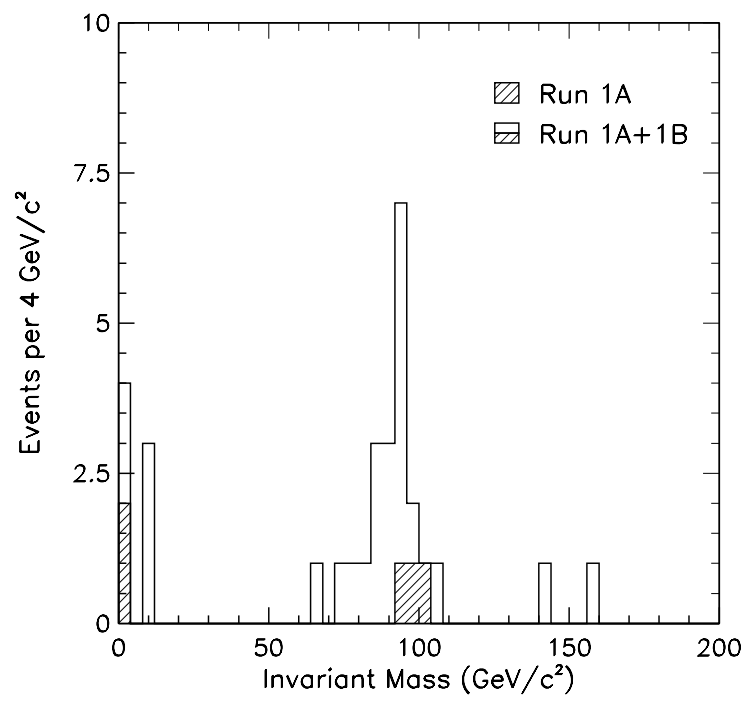

Figure 3.6: The invariant mass distributions of the events removed from the $W / W^{\prime} \rightarrow$ $\mu \nu$ event candidate sample by the $Z / \gamma \rightarrow \mu \mu$ rejection cut.

when a second muon candidate was identified. The second muon candidate was required to have $p_{T}>15 \mathrm{GeV} / c, E_{\text {had }}<6 \mathrm{GeV}, E_{\text {em }}<2 \mathrm{GeV}, E_{\text {had }}+E_{\text {em }}>0.1 \mathrm{GeV}$, Iso $<3 \mathrm{GeV}$, and to satisfy the $\Delta x$ matching requirements. Also, the difference between $z_{0}$ 's of the two muon candidates was required to satisfy $\left|\Delta z_{0}\right|<5 \mathrm{~cm}$. The events that satisfied these requirements were removed regardless of the invariant mass of the dimuon system. The invariant mass distribution of the rejected events is shown in Figure 3.6. A small $Z \rightarrow \mu \mu$ signal is evident.

\subsection{Cosmic Ray Rejection}

A cosmic ray can be misidentified as a $W / W^{\prime} \rightarrow \mu \nu$ event when one of the two resulting muon candidates is identified as a primary muon candidate whereas the other is not reconstructed or identified as a muon candidate. Two independent methods were used to remove cosmic ray events.

Since cosmic rays pass the detector at random time intervals, the timing information relative to the beam bunch crossing can be used to reject events. The hadron TDCs, $t_{\text {had }}$, record the time intervals between the signal from the phototubes of each hadron calorimeter tower and the "common stop" that is issued $\sim 550$ ns after the $p \bar{p}$ 

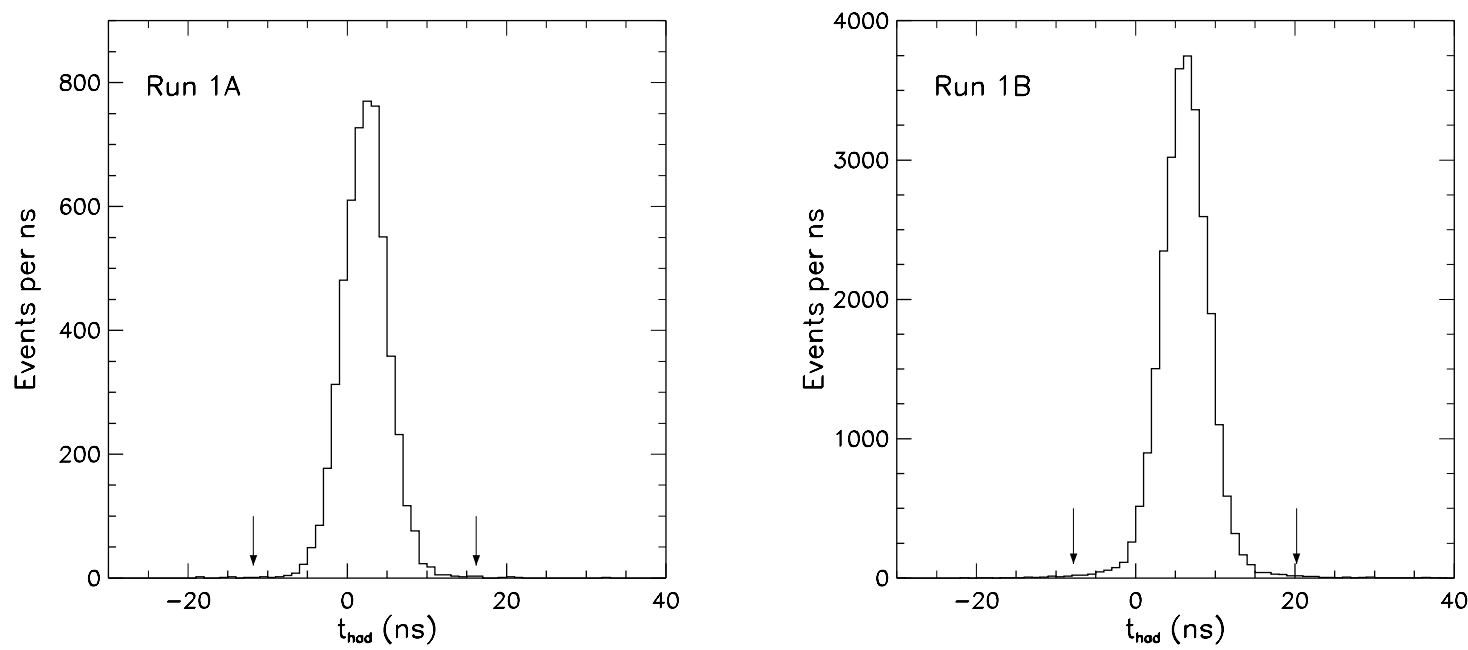

Figure 3.7: The hadron TDC, $t_{\text {had }}$, distributions of the $W / W^{\prime} \rightarrow \mu \nu$ event candidates in Run 1A (left) and Run 1B (right). All the cuts were applied except $t_{\text {had }}$ cuts. The arrows indicate where the cuts were made.

collision. ${ }^{7}$ The primary muon candidate was required to satisfy $\left|t_{\text {had }}-2.2\right|<14.0 \mathrm{~ns}$ for Run $1 \mathrm{~A}$ and $\left|t_{\text {had }}-6.2\right|<14.0 \mathrm{~ns}$ for Run 1B. Figure 3.7 shows the $t_{\text {had }}$ distributions of the $W / W^{\prime} \rightarrow \mu \nu$ event candidates without the $t_{\text {had }}$ requirement.

Cosmic rays were also removed using the event topology information. A fraction of cosmic rays that leave the obvious signatures, two back-to-back muon candidates, was removed by the $Z / \gamma \rightarrow \mu \mu$ rejection cut described in Section 3.6. In the case where one of the muon candidates fails the muon identification criteria, cosmic rays could end up in the $W / W^{\prime} \rightarrow \mu \nu$ event candidate sample. Two methods were used to reject most of the remaining background events. The first method removed cosmic rays that left a CTC track opposite the primary muon candidate. Events were rejected if there was a CTC track with $p_{T}>15 \mathrm{GeV} / c$ that satisfied ||$\phi_{1}-\phi_{2}|-\pi|<0.05$ and $\left|\eta_{1}+\eta_{2}\right|<0.2$, where $\phi_{1}\left(\phi_{2}\right)$ and $\eta_{1}\left(\eta_{2}\right)$ are the azimuthal angle and the pseudorapidity of the primary muon candidate (CTC track), respectively. Events

\footnotetext{
${ }^{7}$ The $t_{\text {had }}$ was not the absolute time interval between the signal from the hadronic calorimeter and the common stop signal, however. A shift was added that centered the TDC around zero for beam related signals.
} 


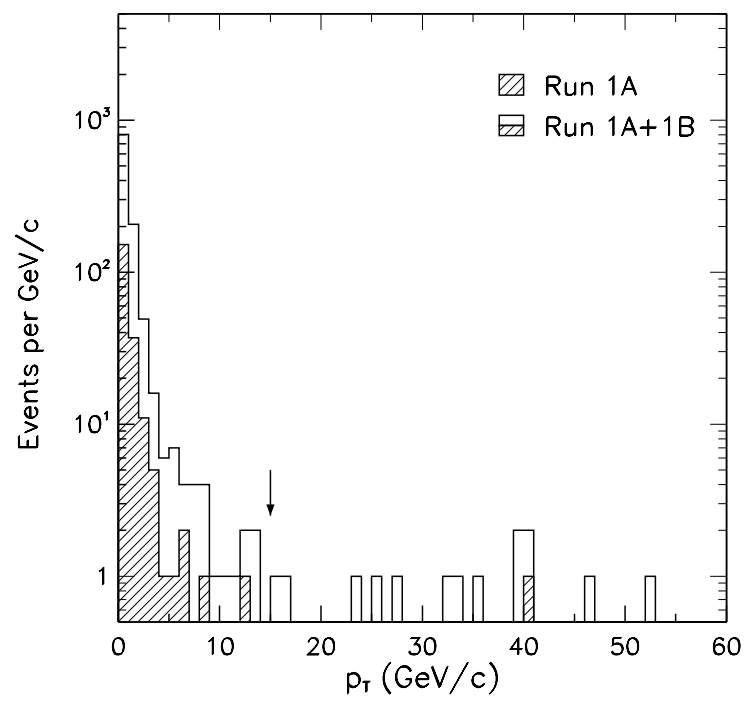

Figure 3.8: The transverse momentum distributions of the "back-to-back" tracks. All the cuts were applied except the "back-to-back" track removal. The arrow indicates where the cut was made.

were also removed if there was a muon stub in either the CMU or CMP that satisfied ||$\phi_{1}-\phi_{2}|-\pi|<0.05$, where $\phi_{1}$ and $\phi_{2}$ are the azimuthal angles of the primary muon candidate and a muon stub, respectively. The muon stubs in the CMX were not used here since the CMX was found to generate too many "fake" muon stubs. The transverse momentum distribution of the "back-to-back" CTC tracks and the opening angle distribution between the primary muon candidate and the muon stub farthest from the primary muon candidate in $\phi$ are shown in Figure 3.8 and 3.9, respectively.

These two cuts are referred to as "back-to-back" cuts.

\subsection{Final $W / W^{\prime} \rightarrow \mu \nu$ Candidate Sample}

There were 5408 Run $1 \mathrm{~A}$ events and 26584 Run 1B events in the $W / W^{\prime} \rightarrow \mu \nu$ event candidate sample. The transverse mass distributions are shown in Figure 3.10. There were one event and 13 events above $M_{T}=200 \mathrm{GeV} / c^{2}$ in Run $1 \mathrm{~A}$ and Run $1 \mathrm{~B}$, respectively, as listed in Table 3.1. The event display of the $W / W^{\prime} \rightarrow \mu \nu$ candidate event with the highest transverse mass value $\left(=403_{-65}^{+96} \mathrm{GeV} / c^{2}\right)$ is shown in Figure 3.11.

There is no clear excess of events in the high transverse mass region. In order 


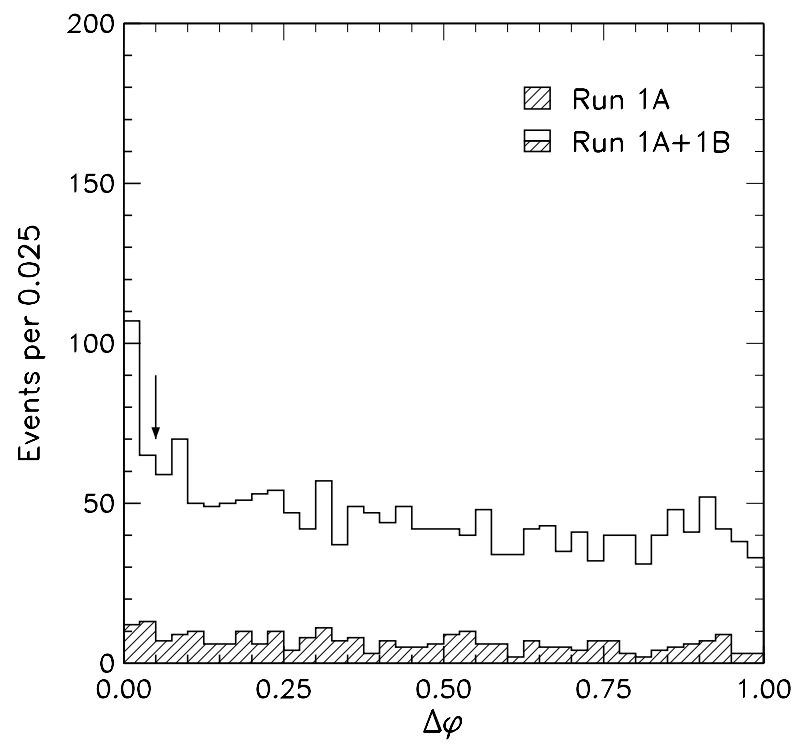

Figure 3.9: The opening angle between the primary muon candidate and the muon stub farthest from the primary muon candidate in $\phi$. The arrow indicates where the cut was made.

to quantify this observation, the efficiency for detecting a $W^{\prime} \rightarrow \mu \nu$ event must be determined relative to the efficiency for detecting a $W \rightarrow \mu \nu$ event, and the expected transverse mass distribution for the sources contributing to the event sample must be determined. These are determined in the following two chapters. 


\begin{tabular}{|c|rrr|}
\hline Run & $p_{T}^{\mu}(\mathrm{GeV} / c)$ & ${ }_{T}(\mathrm{GeV})$ & $M_{T}\left(\mathrm{GeV} / c^{2}\right)$ \\
\hline \hline 1A & $121_{-11}^{+13}$ & $98_{-11}^{+13}$ & $218_{-22}^{+26}$ \\
\hline 1B & $98_{-9}^{+11}$ & $123_{-10}^{+12}$ & $214_{-18}^{+22}$ \\
& $190_{-31}^{+45}$ & $84_{-37}^{+45}$ & $246_{-64}^{+94}$ \\
& $145_{-18}^{+25}$ & $145_{-19}^{+25}$ & $290_{-37}^{+50}$ \\
& $113_{-12}^{+15}$ & $111_{-12}^{+15}$ & $224_{-23}^{+29}$ \\
& $119_{-13}^{+16}$ & $96_{-13}^{+17}$ & $214_{-26}^{+33}$ \\
& $73_{-5}^{+6}$ & $156_{-}^{+8}$ & $213_{-11}^{+12}$ \\
& $118_{-12}^{+16}$ & $123_{-13}^{+16}$ & $241_{-25}^{+32}$ \\
& $313_{-75}^{+145}$ & $54_{-54}^{+145}$ & $258_{-115}^{+343}$ \\
& $114_{-12}^{+15}$ & $115_{-12}^{+15}$ & $230_{-24}^{+30}$ \\
& $132_{-15}^{+20}$ & $119_{-16}^{+20}$ & $250_{-31}^{+40}$ \\
& $107_{-10}^{+13}$ & $107_{-11}^{+13}$ & $213_{-21}^{+26}$ \\
& $136_{-16}^{+22}$ & $121_{-17}^{+22}$ & $256_{-33}^{+43}$ \\
& $196_{-32}^{+48}$ & $208_{-33}^{+48}$ & $403_{-65}^{+96}$ \\
\hline
\end{tabular}

Table 3.1: List of candidate events with $M_{T}>200 \mathrm{GeV} / c^{2}$. 


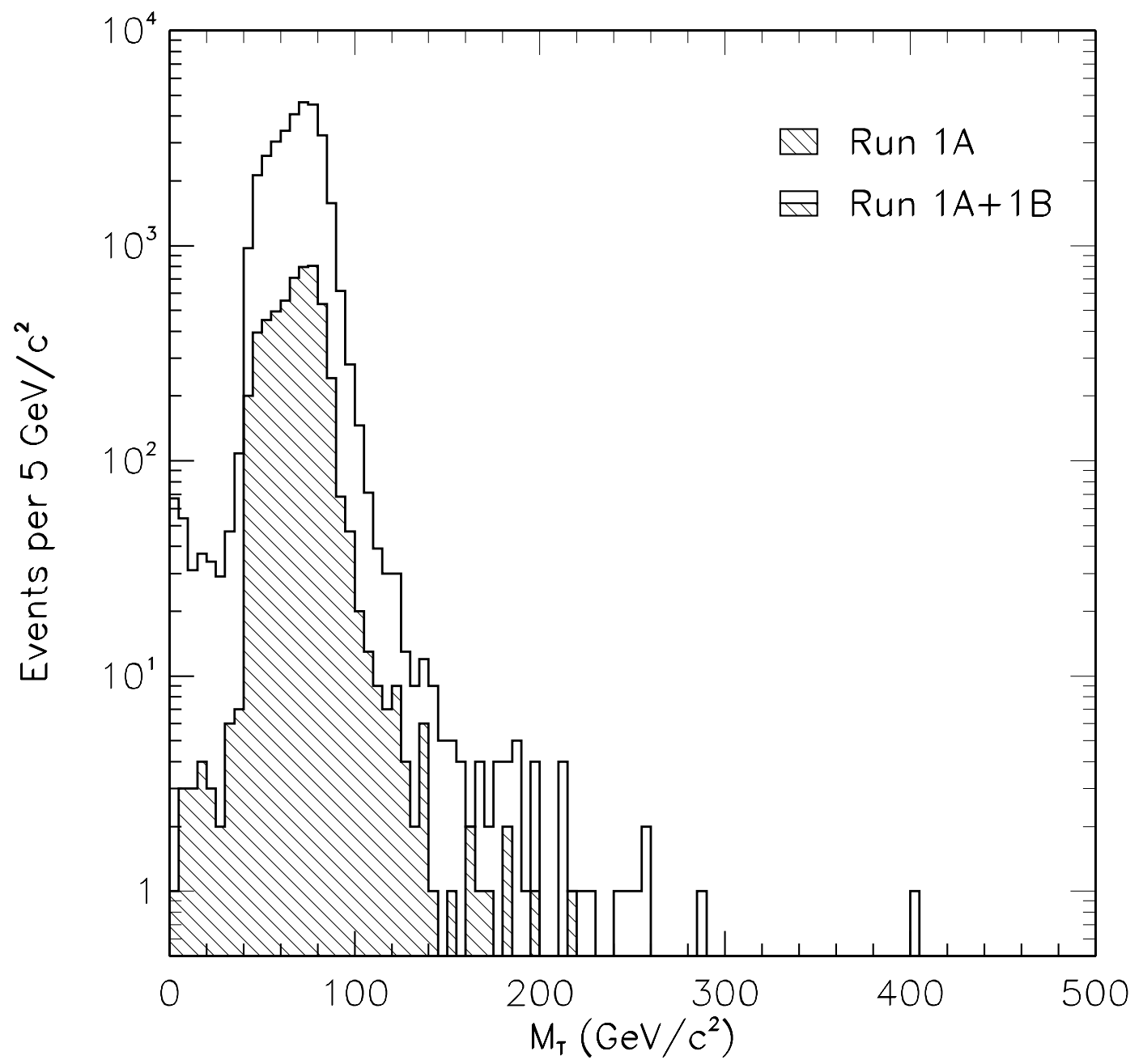

Figure 3.10: The transverse mass distributions of the $W / W^{\prime} \rightarrow \mu \nu$ event candidates in Run 1A and Run 1B. There are 5408 and 26584 events in Run $1 \mathrm{~A}$ and Run 1B, respectively. 

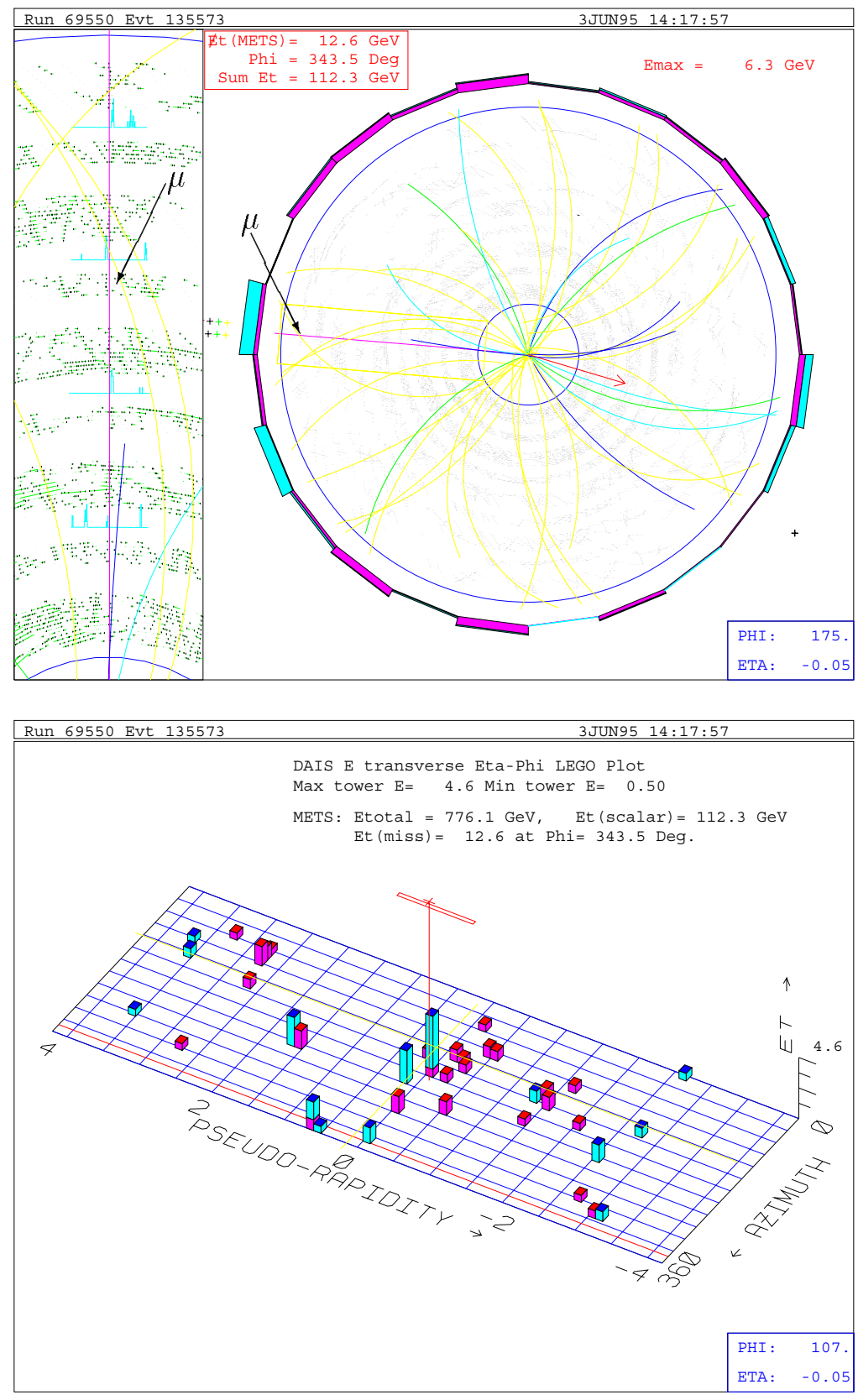

Figure 3.11: The event display of the highest transverse mass $\left(=403_{-65}^{+96} \mathrm{GeV} / \mathrm{c}^{2}\right)$ $W / W^{\prime} \rightarrow \mu \nu$ event candidate. The top figure shows the CTC tracks. The CTC sense wire hits are indicated as dots and the reconstructed tracks are shown as smooth curves. The bars outside the CTC represents transverse energy deposited in the $\mathrm{CEM} / \mathrm{CHA}$ wedges. The missing $E_{T}$ shown near the top of the figure is not corrected for muon candidates. The bottom figure is a "lego" display of energy deposit in the calorimeters. The calorimeter tower associated with the primary muon candidate is marked with a vertical bar. 


\section{Chapter 4}

\section{Efficiencies}

One of the factors in determining the expected number of observed $W^{\prime} \rightarrow \mu \nu$ events in the $W / W^{\prime} \rightarrow \mu \nu$ event candidate sample is the product of the efficiencies of event selection, including triggers, and the kinematic and geometric acceptance of the event. However, since the expected number of $W^{\prime} \rightarrow \mu \nu$ events is normalised to the number of observed $W \rightarrow \mu \nu$ events in the sample, it is the ratio of these products for the $W^{\prime} \rightarrow \mu \nu$ production to $W \rightarrow \mu \nu$ production that is important (see Section 7.1). Effects that are in principle common to the efficiencies or acceptances cancel in the ratio. One therefore can ignore these common effects and only determine the relative size of those effects that do not cancel.

The efficiencies were measured for Run 1B muon candidates, and were then extrapolated for Run 1A muons from the number of observed $W \rightarrow \mu \nu$ events in Run 1A. This technique, detailed in Section 7.2, was employed because the differences in efficiencies between Run $1 \mathrm{~A}$ and $1 \mathrm{~B}$ were modest.

The measured efficiencies of the trigger and event selection are presented in this chapter. Also, the corrections to the event selection efficiencies that depend on the muon momentum are discussed. The efficiencies presented here were incorporated into the detector model described in Section 5.2. 


\begin{tabular}{|cc|c|c|}
\hline \multicolumn{2}{|c|}{ Trigger } & Efficiency & Prescale \\
\hline \hline & CMUP & $0.861_{-0.035}^{+0.029}$ & - \\
Level $1 \cdot 2$ & CMNP & $0.871_{-0.061}^{+0.061}$ & 0.40 \\
& CMX & $0.696_{-0.049}^{+0.045}$ & 0.43 \\
\hline Level 3 & $0.970_{-0.007}^{+0.006}$ & - \\
\hline
\end{tabular}

Table 4.1: Trigger efficiencies in Run 1B measured in Ref. [39].

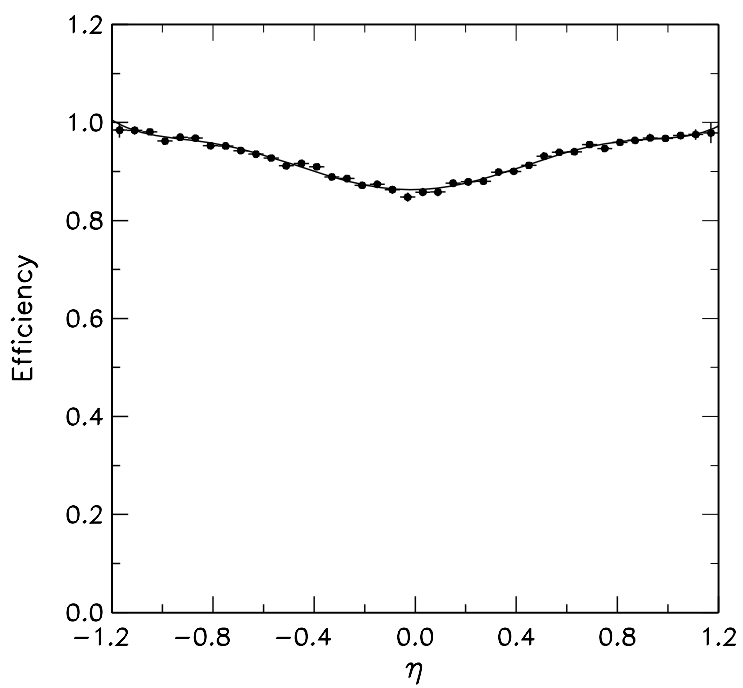

Figure 4.1: CFT efficiency as a function of $\eta$ measured in Ref. [40]. The solid line is a fit to the distribution with a 6 th order polynomial.

\subsection{Trigger Efficiencies}

The efficiency of the Level 1 and Level 2 triggers depended on the CFT tracking and the muon chamber efficiencies. In addition, the CMX trigger efficiency also depended on the scintillator coincidence efficiency (see Section 2.5.3). The trigger efficiencies in Run 1B were determined in Ref. [39] and are shown in Table 4.1. Figure 4.1 shows the CFT tracking efficiency in Run 1B measured in Ref. [40] as a function of $\eta$. The Run 1A CFT tracking efficiency shows a similar $\eta$-dependence [41]. In Run 1A, the Level 1 and Level 2 combined efficiency of the CMUP and CMNUP triggers was measured to be $0.887_{-0.018}^{+0.015}$ in Ref. [42]. The CMUNP trigger, the CMNP equivalent in Run $1 \mathrm{~A}$, was not prescaled. 


\subsection{Muon Selection Efficiencies}

The efficiencies of the muon selection cuts were measured with a sample of $Z \rightarrow \mu \mu$ event candidates. The events were required to have at least one muon candidate that passed all the primary muon selection cuts. ${ }^{1}$ The efficiency of a selection cut was determined from examining whether or not the second muon candidate passed the selection cut in question after it passed all the other selection cuts. ${ }^{2}$

Two methods were used to measure the efficiencies. One method used the "likedetector" muon candidates where both muon candidates hit the same type of muon chambers, that is, either $\mathrm{CMU} / \mathrm{P}-\mathrm{CMU} / \mathrm{P}$ or $\mathrm{CMX}-\mathrm{CMX}{ }^{3}$ Since the muon selection cut efficiencies for the "like-detector" muon candidates would be the same, the efficiency of a cut was calculated as

$$
\varepsilon=\frac{2 N_{2} / N_{1}}{1+N_{2} / N_{1}}
$$

where $N_{1}$ was the number of events that have at least one muon candidate passing the cut and $N_{2}$ was the number of events that had both muon candidates passing the cut.

The second method used the "unlike-detector" muon candidates where one of the muon candidates hit $\mathrm{CMU} / \mathrm{P}$ and the other hit $\mathrm{CMX}$ and vice versa. In this case, the efficiency of a selection cut for a $\mathrm{CMU} / \mathrm{P}$ muon was calculated as $\varepsilon=N_{2} / N_{1}$, where $N_{1}$ was the number of events that had a muon candidate hitting the CMX and passing all cuts and $N_{2}$ was the subset of those events that had a muon candidate that passed the cut in the CMU/P. Since only one muon is required to pass all the cuts in either CMU/P or CMX, the analogous efficiency can be calculated for a CMX muon selection cut as $\varepsilon=N_{2} / N_{1}$, where $N_{1}$ was the number of events that had a muon candidate hitting the $\mathrm{CMU} / \mathrm{P}$ and passing all cuts and $N_{2}$ was the subset of

\footnotetext{
${ }^{1}$ This sample was obtained from a similar set of Level 3 triggers but with no $E_{h a d}<6 \mathrm{GeV}$ requirement.

${ }^{2}$ In principle, the second muon candidate should be required to fire the trigger as well. However, because the trigger requirements were much looser than the muon selection cuts, the bias was expected to be negligible.

${ }^{3}$ Here CMU/P refers to either the CMU only or both the CMU and CMP.
} 


\begin{tabular}{|c|cc|cc|}
\hline Cut & \multicolumn{2}{|c|}{ Like-Detector } & \multicolumn{2}{c|}{ Unlike-Detector } \\
& $N_{2} / N_{1}$ & Efficiency & $N_{2} / N_{1}$ & Efficiency \\
\hline \hline$I s o<3 \mathrm{GeV}$ & $659 / 714$ & $0.9599 \pm 0.0054$ & $489 / 510$ & $0.9588 \pm 0.0088$ \\
$E_{\text {had }}+E_{\text {em }}>0.1 \mathrm{GeV}$ & $659 / 659$ & $1.000_{-0.0010}^{+0.0}$ & $489 / 489$ & $1.000_{-0.0026}^{+0.0}$ \\
$E_{\text {had }}<6 \mathrm{GeV}$ & $659 / 678$ & $0.9858 \pm 0.0033$ & $489 / 496$ & $0.9859 \pm 0.0053$ \\
$E_{\text {em }}<2 \mathrm{GeV}$ & $659 / 697$ & $0.9720 \pm 0.0045$ & $489 / 499$ & $0.9800 \pm 0.0063$ \\
$|\Delta x|$ & $659 / 674$ & $0.9887 \pm 0.0029$ & $489 / 494$ & $0.9899 \pm 0.0045$ \\
$\left|d_{0}\right|$ & $659 / 703$ & $0.9677 \pm 0.0049$ & $489 / 505$ & $0.9683 \pm 0.0078$ \\
$n_{\text {hit }} \geq 30$ & $659 / 665$ & $0.9955 \pm 0.0019$ & $489 / 490$ & $0.9980 \pm 0.0020$ \\
\hline
\end{tabular}

Table 4.2: Efficiencies of event selection cuts for CMU/P muons in CMU/P-CMU/P (like-detector) and CMU/P-CMX (unlike-detector) $Z \rightarrow \mu \mu$ event candidates.

those events that had a muon candidate that passed the cut in the CMX.

Since the two methods used mutually exclusive samples, the measured efficiencies were combined to reduce the uncertainties. The results of the muon selection efficiency measurements are shown in Tables 4.2 through 4.4 .

The efficiency of the $\left|z_{0}-z_{p v}\right|<5 \mathrm{~cm}$ cut was measured using only the primary muon candidates since the selection criteria for the primary vertex only demanded a vertex of class 10 or higher that was closest to the primary muon candidate in the $z$ direction. The $Z \rightarrow \mu \mu$ event candidate sample for this efficiency calculation was required to have both muon candidates passing all the primary muon selection cuts, but not requiring $\left|z_{0}-z_{p v}\right|<5 \mathrm{~cm}$ and not requiring their $z_{0}$ difference to be less than $5 \mathrm{~cm}$. The efficiency was calculated as $\varepsilon=N_{2} / N_{1}$, where $N_{1}$ is the number of events in this particular $Z \rightarrow \mu \mu$ event candidate sample and $N_{2}$ is the subset of those with the primary muon candidate passing the cut. The results are shown in Table 4.5.

The effects of the $\left|z_{0}\right|<60 \mathrm{~cm}, r_{\text {exit }}>132 \mathrm{~cm}$, and $p_{T}>20 \mathrm{GeV} / c$ cuts were incorporated in the acceptance calculation (see Section 5.2). 


\begin{tabular}{|c|cc|cc|}
\hline Cut & \multicolumn{2}{|c|}{ Like-Detector } & \multicolumn{2}{c|}{ Unlike-Detector } \\
& $N_{2} / N_{1}$ & Efficiency & $N_{2} / N_{1}$ & Efficiency \\
\hline \hline$I s o<3 \mathrm{GeV}$ & $83 / 88$ & $0.9708 \pm 0.0131$ & $489 / 513$ & $0.9532 \pm 0.0093$ \\
$E_{\text {had }}+E_{\text {em }}>0.1 \mathrm{GeV}$ & $83 / 83$ & $1.000_{-0.0078}^{+0.0}$ & $489 / 489$ & $1.000_{-0.0026}^{+0.0}$ \\
$E_{\text {had }}<6 \mathrm{GeV}$ & $83 / 87$ & $0.9765 \pm 0.0118$ & $489 / 504$ & $0.9702 \pm 0.0076$ \\
$E_{\text {em }}<2 \mathrm{GeV}$ & $83 / 90$ & $0.9595 \pm 0.0153$ & $489 / 506$ & $0.9664 \pm 0.0080$ \\
$|\Delta x|$ & $83 / 84$ & $0.9940 \pm 0.0060$ & $489 / 495$ & $0.9879 \pm 0.0049$ \\
$\left|d_{0}\right|$ & $83 / 85$ & $0.9881 \pm 0.0084$ & $489 / 499$ & $0.9800 \pm 0.0063$ \\
$n_{\text {hit }} \geq 30$ & $83 / 87$ & $0.9765 \pm 0.0118$ & $489 / 499$ & $0.9800 \pm 0.0063$ \\
\hline
\end{tabular}

Table 4.3: Efficiencies of event selection cuts for CMX muons in CMX-CMX (likedetector) and CMX-CMU/P (unlike-detector) $Z \rightarrow \mu \mu$ event candidates.

\begin{tabular}{|c|cc|}
\hline Cut & \multicolumn{2}{|c|}{ Efficiency } \\
& CMU $/ \mathrm{P}$ & CMX \\
\hline \hline Iso $<3 \mathrm{GeV}$ & $0.9596 \pm 0.0046$ & $0.9591 \pm 0.0076$ \\
$E_{\text {had }}+E_{\text {em }}>0.1 \mathrm{GeV}$ & $1.000_{-0.0009}^{+0.0}$ & $1.000_{-0.0025}^{+0.0}$ \\
$E_{\text {had }}<6 \mathrm{GeV}$ & $0.9858 \pm 0.0028$ & $0.9721 \pm 0.0067$ \\
$E_{\text {em }}<2 \mathrm{GeV}$ & $0.9747 \pm 0.0037$ & $0.9649 \pm 0.0071$ \\
$|\Delta x|$ & $0.9891 \pm 0.0024$ & $0.9903 \pm 0.0038$ \\
$\left|d_{0}\right|$ & $0.9679 \pm 0.0041$ & $0.9829 \pm 0.0050$ \\
$n_{\text {hit }} \geq 30$ & $0.9966 \pm 0.0014$ & $0.9792 \pm 0.0055$ \\
\hline
\end{tabular}

Table 4.4: Combined efficiencies of the cuts on CMU/P and CMX muons.

\begin{tabular}{|c|cc|}
\hline Muon Type & $N_{2} / N_{1}$ & Efficiency \\
\hline \hline CMU $/ \mathrm{P}$ & $904 / 960$ & $0.9417 \pm 0.0076$ \\
CMX & $330 / 350$ & $0.9428 \pm 0.0124$ \\
\hline
\end{tabular}

Table 4.5: Efficiencies of the matching cut between the track vertex $z$ position and the primary vertex $z$ position $\left(\left|z_{0}-z_{p v}\right|<5 \mathrm{~cm}\right)$. 


\section{3 $Z / \gamma \rightarrow \mu \mu$ and Cosmic Ray Rejection Effi- ciency}

The efficiency of the $Z / \gamma \rightarrow \mu \mu$ rejection cut was estimated using $W / W^{\prime} \rightarrow \mu \nu$ event candidates that passed all the event selection cuts but failed the $Z / \gamma \rightarrow \mu \mu$ rejection cut. As shown in Figure 3.6, there were a total of 25 such events in the Run 1B sample with 18 events within the $Z$ boson mass window, $61<M<121 \mathrm{GeV} / c^{2}$. Assuming the 7 events outside the mass window had come from $W$ +jets production and the 18 events within the mass window had come from $Z / \gamma \rightarrow \mu \mu$ decays, the efficiency of the cut is the number of $W / W^{\prime} \rightarrow \mu \nu$ event candidates passing all the cuts divided by the sum of this number and the number of events that failed the $Z / \gamma \rightarrow \mu \mu$ rejection cut that were outside the $Z$ boson mass window. This efficiency was $0.9997 \pm 0.0001$.

The efficiency of the timing cut, $\left|t_{\text {had }}-6.2\right|<14.0 \mathrm{~ns}$, was measured with the $Z \rightarrow$ $\mu \mu$ sample using the same methods discussed in Section 4.2. For the CMU/P muon, the efficiencies were $0.9578 \pm 0.0055\left(N_{2} / N_{1}=659 / 717\right)$ and $0.9626 \pm 0.0084\left(N_{2} / N_{1}=\right.$ 489/508) for "like-detector" muon candidates and "unlike-detector" muon candidates, respectively, giving the combined efficiency of $0.9593 \pm 0.0046$. For the CMX muon, the efficiencies were $0.8137 \pm 0.0297\left(N_{2} / N_{1}=83 / 121\right)$ and $0.8519 \pm 0.0148\left(N_{2} / N_{1}=\right.$ 489/574) for "like-detector" muon candidates and "unlike-detector" muon candidates, respectively, giving the combined efficiency of $0.8442 \pm 0.0133$.

The "back-to-back" cuts (see Section 3.7) were correlated with each other. Thus, their combined efficiency was measured using a " $W \rightarrow \mu \nu$ sample" that passed both "back-to-back" cuts, a " $W \rightarrow \mu \nu$ sample" that failed either "back-to-back" cuts, and a cosmic ray candidate sample. Here, the " $W \rightarrow \mu \nu$ sample" was defined as those events that passed all cuts for the final $W / W^{\prime} \rightarrow \mu \nu$ event candidate sample but not requiring the cosmic ray cuts and the $Z / \gamma \rightarrow \mu \mu$ rejection cut. It consisted of 26746 events. The cosmic ray candidates were defined as those events that failed both "back-to-back" cuts, and satisfied $\left|d_{0}\right|>0.2 \mathrm{~cm}$ and $\left|z_{0}-z_{p v}\right|>10 \mathrm{~cm}$ instead of the muon selection cuts on the same parameters. Also, the $\left|t_{\text {had }}-6.2\right|<14$ ns cut was not applied.

There were 176 events in the " $W \rightarrow \mu \nu$ sample" that failed either back-to-back 


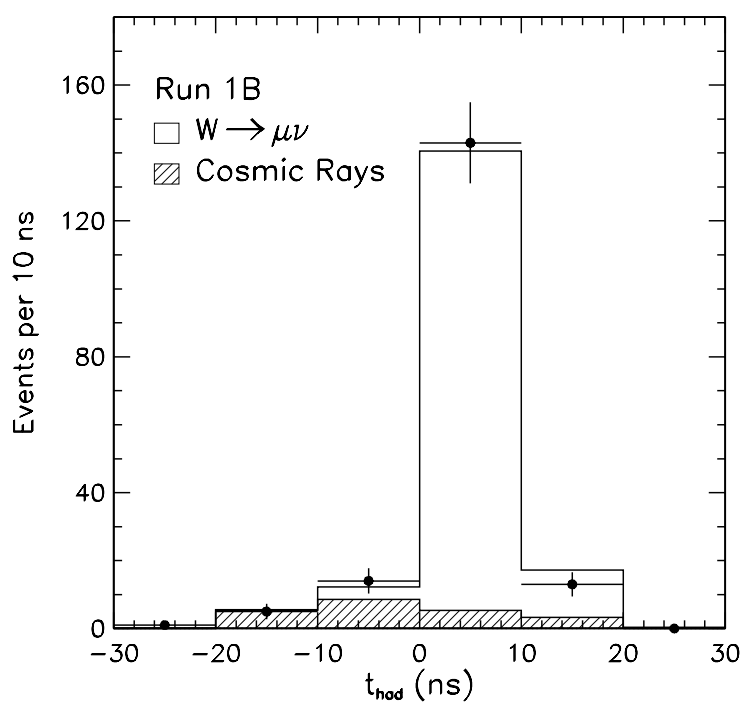

Figure 4.2: The hadron TDC, $t_{\text {had }}$, distribution of the events that failed the "backto-back" cuts (dots) is fitted with that of the $W \rightarrow \mu \nu$ sample and the cosmic ray sample in Run 1B.

cuts in the whole hadron TDC region and 169 events that were inside the hadron TDC cut window $\left(\left|t_{\text {had }}-6.2\right|<14.0 \mathrm{~ns}\right)$. The 176 event sample consisted of real $W \rightarrow \mu \nu$ events and cosmic rays, and these could be statistically separated using the $t_{\text {had }}$ distribution. The $t_{\text {had }}$ distribution of those 176 events were fitted with that of cosmic ray candidates and the " $W \rightarrow \mu \nu$ sample" that passed both "back-to-back" cuts. The fit, shown in Figure 4.2, estimated the number of non-cosmic ray events within the hadron TDC cut window to be $153 \pm 13$. This, therefore, represents the inefficiency of this cut, assuming that the composition of these 153 events was the same as the final $W / W^{\prime} \rightarrow \mu \nu$ event candidate sample. ${ }^{4}$ The efficiency calculated was $0.9943 \pm 0.0005$.

Although the $Z / \gamma \rightarrow \mu \mu$ rejection cut and the "back-to-back" cuts were correlated, the correlation was found to be small. The efficiencies of the $Z / \gamma \rightarrow \mu \mu$ rejection cut and the cosmic ray rejection cuts are summarised in Table 4.6.

\footnotetext{
${ }^{4}$ The second largest contribution to this sample would be from $Z / \gamma \rightarrow \mu \mu$. The contribution was estimated to be $18 \pm 4$ using a sample of $Z / \gamma \rightarrow \mu \mu$ Monte Carlo events, thus supporting the assumption that the 153 event candidate sample consisted primarily of $W / W^{\prime} \rightarrow \mu \nu$ events.
} 


\begin{tabular}{|c|c|}
\hline Cut & Efficiency \\
\hline \hline$Z / \gamma \rightarrow \mu \mu$ Rejection & $0.9997 \pm 0.0001$ \\
$\left|t_{\text {had }}-6.2\right|<14.0 \mathrm{~ns}$ & $0.9588 \pm 0.0037(\mathrm{CMU} / \mathrm{P})$ \\
back-to-back cuts & $0.8419 \pm 0.0127(\mathrm{CMX})$ \\
\hline
\end{tabular}

Table 4.6: Efficiencies of the $Z / \gamma \rightarrow \mu \mu$ rejection cut and cosmic ray rejection cuts.

\subsection{Efficiency Correction for Bremsstrahlung}

Because the muons from $W^{\prime} \rightarrow \mu \nu$ decay are expected to have significantly higher momenta than those from the $Z \rightarrow \mu \mu$ process, the efficiencies of the selection cuts that depend on the muon momentum need to be corrected for momentum-dependent effects. The two most significant sources of such muon momentum-dependence involved the amount of energy deposited in the calorimeters by a muon and the final state radiation of photons off a muon. These were both taken into account in the efficiency calculation.

At the muon momenta of interest, $p \gtrsim 100 \mathrm{GeV} / c$, energy loss in matter by radiative processes becomes important and this energy loss can no longer be treated as independent of the muon momentum. The momentum-dependence of the efficiency of the minimum ionisation cuts ( $E_{\text {had }}<6 \mathrm{GeV}$ and $E_{e m}<2 \mathrm{GeV}$ ) had been studied in Ref. [43] using a detailed GEANT Monte Carlo calculation [44] and this dependence is shown in Figure 4.3. The efficiency curve was scaled to match the measured efficiency of the minimum ionisation cuts at the mean momenta of muon candidates in the $Z \rightarrow \mu \mu$ event candidate sample,$^{5}$ and half the differences between the curve at the mean momenta of muons and the measured values for the CMU/P and CMX muons were assigned as the systematic uncertainties for the efficiency of the minimum ionisation cuts.

The final state radiation of photons off the muon affects the efficiencies of the isolation cut and minimum ionisation cuts depending on the separation between the muon and photons that were radiated off the muon. The effects of such final state

\footnotetext{
${ }^{5}$ The mean momentum was $47.3 \mathrm{GeV} / c$ for $\mathrm{CMU} / \mathrm{P}$ muon candidates and $56.9 \mathrm{GeV} / c$ for $\mathrm{CMX}$ muon candidates.
} 


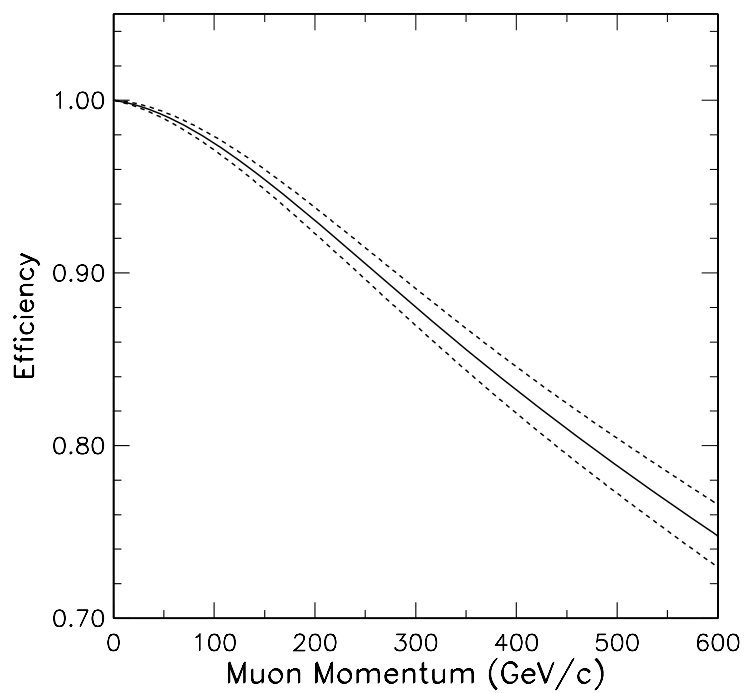

Figure 4.3: The combined efficiency of the minimum ionisation cuts, $E_{\text {had }}<6 \mathrm{GeV}$ and $E_{e m}<2 \mathrm{GeV}$, as a function of muon momentum calculated in Ref. [43]. The solid line is the nominal value and the dashed lines are the $\pm 1 \sigma$ statistical uncertainty variations.

radiation were studied using a PYTHIA Monte Carlo calculation [45] that generated $W \rightarrow \mu \nu$ and $W^{\prime} \rightarrow \mu \nu$ events as described in Section 5.1. The response of the detector to these events was modelled by CDFSIM, a sophisticated GEANT-like simulation of the CDF detector. The correction factor this introduced to the combined efficiency of isolation and minimum ionisation cuts with respect to the $W \rightarrow \mu \nu$ process had to account for the effects of these cuts already incorporated in the efficiency calculation, and was determined using the expression

$$
\text { Correction Factor }=\left[\frac{\left(N_{\gamma 2}+N_{\bar{\gamma} 2}\right)}{\left(N_{\gamma 1}+N_{\bar{\gamma} 1}\right)} \frac{N_{\bar{\gamma} 1}}{N_{\bar{\gamma} 2}}\right]_{W^{\prime}} /\left[\frac{\left(N_{\gamma 2}+N_{\bar{\gamma} 2}\right)}{\left(N_{\gamma 1}+N_{\bar{\gamma} 1}\right)} \frac{N_{\bar{\gamma} 1}}{N_{\bar{\gamma} 2}}\right]_{W},
$$

where $N_{\gamma 1}\left(N_{\bar{\gamma} 1}\right)$ is the number of events that $\operatorname{did}(\operatorname{did}$ not) radiate a photon before the cuts and $N_{\gamma 2}\left(N_{\bar{\gamma} 2}\right)$ is the number of events that did (did not) radiate a photon that passed the cuts. The factor $N_{\bar{\gamma} 1} / N_{\bar{\gamma} 2}$ compensates for the muon momentum dependence on the minimum ionisation cut efficiency that was already accounted for. Figure 4.4 shows the correction factor as a function of the $W^{\prime}$ boson mass. 


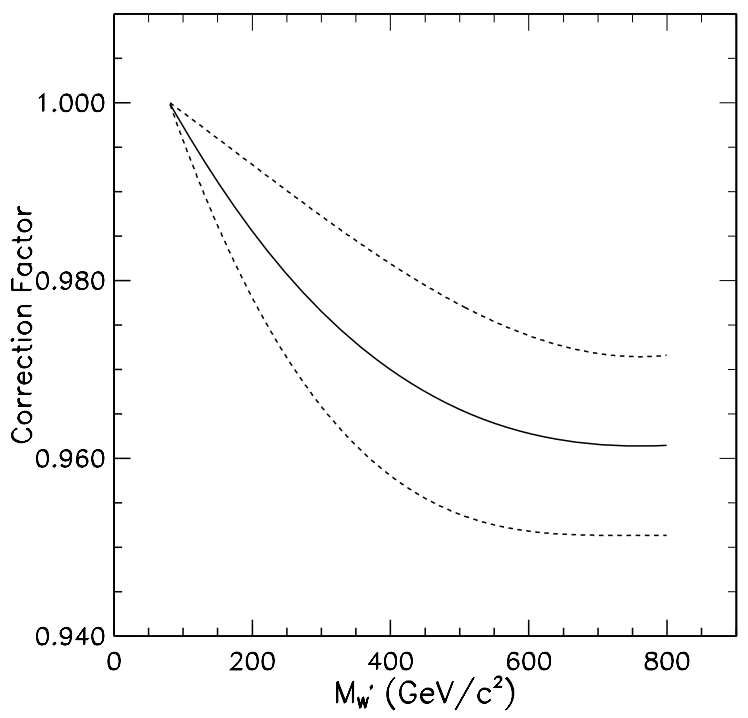

Figure 4.4: The correction factor to the combined efficiency of the isolation and minimum ionisation cuts arising from the effects of final state photon radiation. The dashed lines represent the $\pm 1 \sigma$ uncertainty variations. 


\section{Chapter 5}

\section{Monte Carlo Simulation}

The fraction of $W \rightarrow \mu \nu$ or $W^{\prime} \rightarrow \mu \nu$ events produced in $p \bar{p}$ collisions ending up in the $W / W^{\prime} \rightarrow \mu \nu$ event candidate sample (see Chapter 3 ) and the shape of the transverse mass distribution of these events are determined by the event kinematics, geometrical effects of the detector, and efficiencies of triggers and event selection. These were determined by Monte Carlo calculations. This chapter describes the algorithms used to model $W \rightarrow \mu \nu$ and $W^{\prime} \rightarrow \mu \nu$ events and their detection by the CDF detector.

\section{$5.1 \quad W / W^{\prime} \rightarrow \mu \nu$ Event Generation}

Samples of $W \rightarrow \mu \nu$ and $W^{\prime} \rightarrow \mu \nu$ events were generated with the PYTHIA Monte Carlo programme [45] using the CTEQ4A1 next-to-leading order parton distribution function set [16]. The choice of the CTEQ4A1 parton distribution function set was motivated by the facts that it is one of the latest next-to-leading order parton distribution function sets and it gives median cross section values for $W^{\prime}$ production among the next-to-leading order parton distribution function sets (see Section 7.3.2). Even though the PYTHIA calculation includes the higher order processes for $W$ and $W^{\prime}$ boson production, it was found to be inadequate to describe the $W$ boson $p_{T}$ distribution. Therefore, $d^{2} \sigma / d p_{T} d y$ distributions of $W$ as well as $W^{\prime}$ bosons were calculated separately and used to describe the $W / W^{\prime} \rightarrow \mu \nu$ system. The $d^{2} \sigma / d p_{T} d y$ distributions of $W$ and $W^{\prime}$ bosons were obtained from a next-to-leading order cal- 
culation of P. A. Arnold and R. P. Kauffman [46]. The calculation used soft gluon resummation at low $p_{T}$ and a next-to-leading order perturbative QCD calculation at high $p_{T}$. The $d^{2} \sigma / d p_{T} d y$ distributions were calculated for $p_{T} \leq 200 \mathrm{GeV} / c$ and $|y| \leq 2.875$ for $W$ and $W^{\prime}$ bosons using the CTEQ4A1 parton distribution function set with $\Lambda_{Q C D}^{[4]}=140 \mathrm{MeV}$. Figure 5.1 shows the transverse momentum distributions and the rapidity distributions of $W^{+}$and $W^{+}$bosons of various masses.

The production cross sections for $W$ and $W^{\prime}$ bosons were calculated to the leading order process as described in Section 1.3. To account for higher-order QCD effects, the cross section of the leading order process was multiplied by a $K$-factor

$$
K=1+\frac{8 \pi}{9} \alpha_{s}\left(M_{W^{\prime}}\right)
$$

where $\alpha_{s}\left(M_{W^{\prime}}\right)$ is the strong coupling constant calculated at $Q^{2}=M_{W^{\prime}}^{2}$. The $K$-factor has a typical value of $1.2 \sim 1.3$ at $\sqrt{s}=1.8 \mathrm{TeV}$.

The $W^{\prime}$ bosons were generated with masses from $200 \mathrm{GeV} / c^{2}$ to $800 \mathrm{GeV} / c^{2}$ in $50 \mathrm{GeV} / c^{2}$ intervals. The branching fraction of the $W^{\prime} \rightarrow \mu \nu$ process and the total width of the $W^{\prime}$ boson as a function of $W^{\prime}$ boson mass are shown in Figures 1.4 and 1.5, respectively. The branching fraction of the $W \rightarrow \mu \nu$ process and the total width and the mass of the $W$ boson in these calculations used were $1 / 9$ and $2.04 \mathrm{GeV}$, respectively [47]. The $W$ boson mass used in these calculations was $80.4 \mathrm{GeV} / c^{2}$ [48].

\subsection{Detector Simulation}

The detector simulation consisted of an underlying event model, which accounts for the response of the calorimeter to the overall transverse energy flow associated with the production of a boson, and a detector model, which accounts for the detection efficiency of the muons, trigger efficiencies, and other resolution effects. For each Monte Carlo event, the underlying event was determined, the resolution of the muon candidate was accounted for, and the effects of the trigger and detector acceptance were taken into account. 

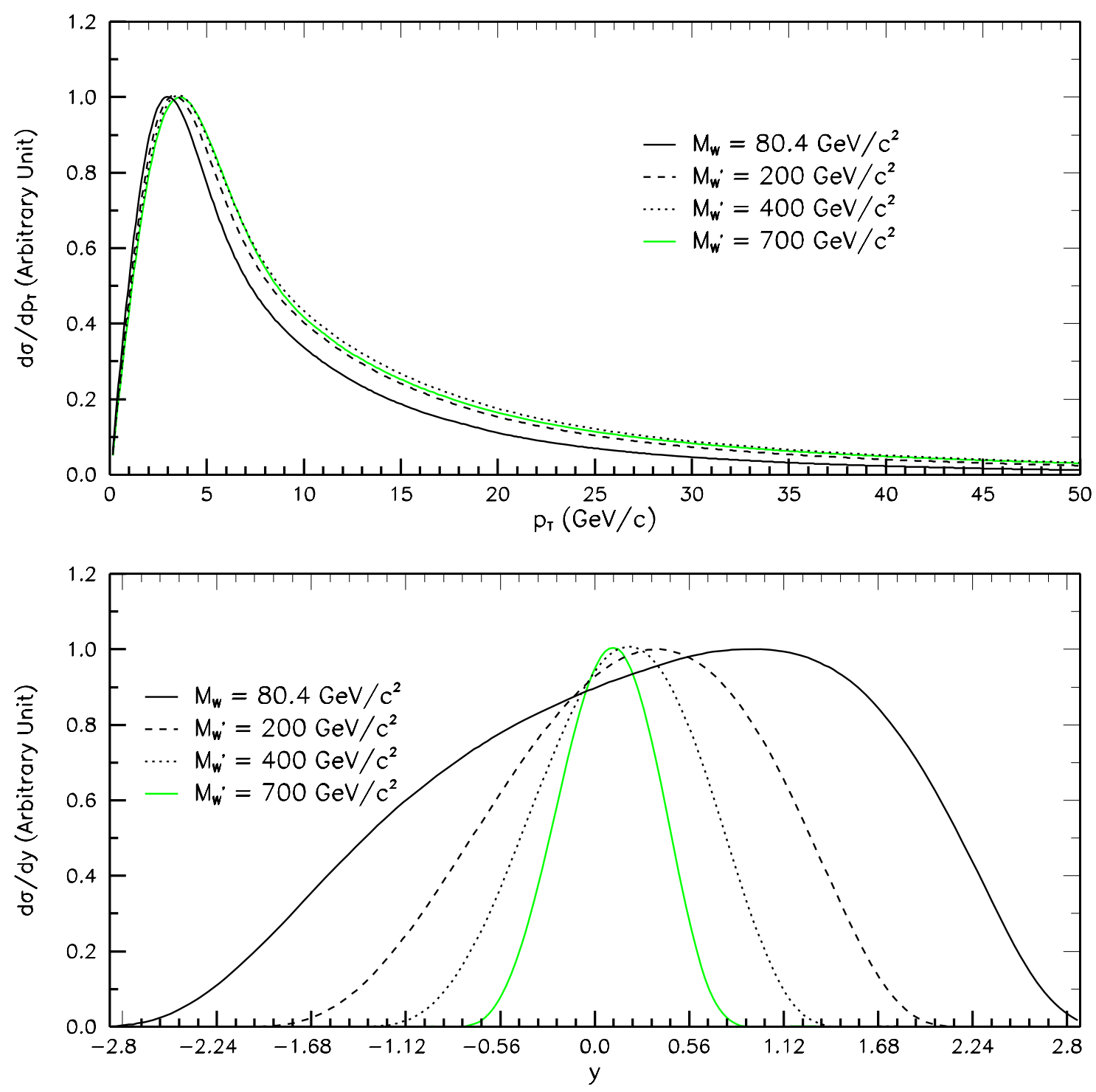

Figure 5.1: The transverse momentum distributions (top) and rapidity distributions (bottom) of the $W^{+}$boson and $W^{\prime+}$ bosons with different masses calculated with a next-to-leading order calculation by P. B. Arnold and R. P. Kauffman [46] using the CTEQ4A1 parton distribution function set. 


\subsubsection{Underlying Event Model}

The transverse momentum of the neutrino from a $W / W^{\prime}$ decay is determined indirectly by the measurement of $\vec{f}_{T}$. The energy flow associated with the underlying event, $\vec{u}$, is defined as the vector sum of the transverse energy flow of the particles that are not the decay products of the boson of interest. Therefore, $\vec{F}_{T}$ is related to the underlying event as $\vec{f}_{T}=\vec{u}-\vec{p}_{T}^{\mu}$, where $\vec{p}_{T}^{\mu}$ is the transverse momentum of the muon from the $W / W^{\prime}$ boson decay. Contributions to the observed underlying event come from the jets produced in association with the boson (the recoiling jets), the remnants of the $p \bar{p}$ collision, the jets from other interactions in the same beam bunch crossing, and any energy mismeasurement in the calorimeter. The recoiling jets are the component that gives non-zero $\vec{u}$ on average whereas other contributions give $\vec{u}$ with mean values of zero. Therefore, $\vec{u}$ is expected to be a function of the boson $p_{T}$, which balances out the recoiling jets. The underlying event can be decomposed into two components; $u_{L}$, the component longitudinal to the direction of the muon from $W / W^{\prime}$ boson, and $u_{T}$, the component perpendicular to that of the muon. The transverse mass can be written as

$$
M_{T}=2 \sqrt{p_{T}^{\mu}\left(p_{T}^{\mu}+u_{L}\right)}
$$

and, therefore, only $u_{L}$ contributes to the transverse mass determination.

The many contributions to the observed characteristics of the underlying event make it difficult to model it accurately with Monte Carlo calculations. Furthermore, the energy flow in the underlying event cannot be modelled using the $W \rightarrow \mu \nu$ sample, as the $p_{T}$ of the $W$ boson cannot be determined independently of the underlying event. Therefore, samples of $Z \rightarrow \mu \mu$ events were used to model the underlying event with the $Z$ boson $p_{T}$ calculated from its decay muon momenta. If one assumes that the response to the recoiling jets from a $W$ boson of a given $p_{T}$ is similar to that of the recoiling jets from a $Z$ boson of the same $p_{T}[49,50]$, their underlying events are also expected to be similar. In Figure 5.2, the $u_{L}$ and $u_{T}$ distributions of the $W / W^{\prime} \rightarrow \mu \nu$ event candidate sample and a $Z \rightarrow \mu \mu$ sample for Run 1B are compared and shown to agree well with each other. Here, $u_{L}$ and $u_{T}$ for $Z \rightarrow \mu \mu$ events were calculated 

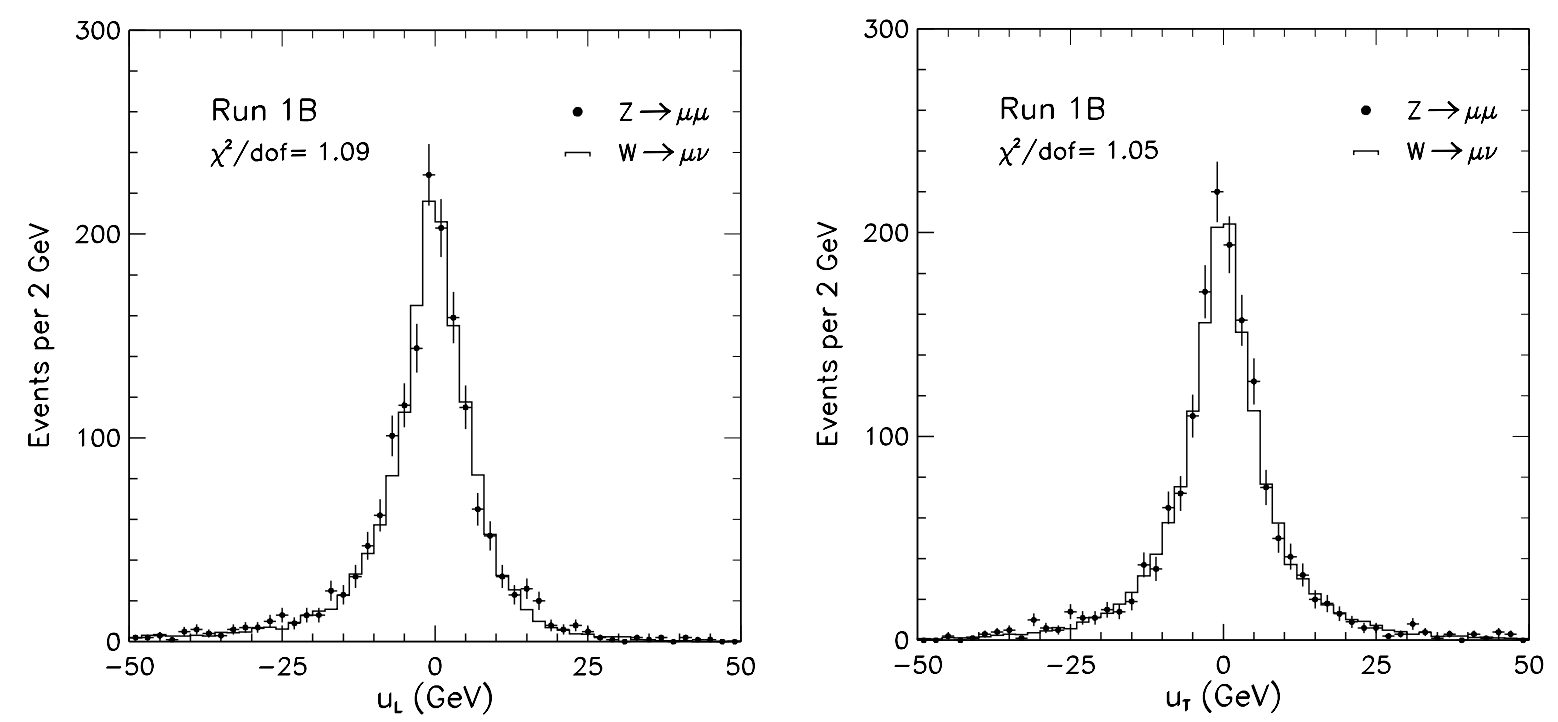

Figure 5.2: Comparisons of $u_{L}$ (left) and $u_{T}$ (right) distributions between the $Z \rightarrow \mu \mu$ sample and the $W / W^{\prime} \rightarrow \mu \nu$ event candidates in Run $1 \mathrm{~B}$.

by randomly choosing one of the muons to be the analogue of the neutrino and the other to be the muon from the corresponding $W \rightarrow \mu \nu$ decay.

The $Z \rightarrow \mu \mu$ samples were made by requiring both muon candidates to satisfy the muon selection criteria used in the $W / W^{\prime} \rightarrow \mu \nu$ event candidate selection criteria. In addition to these muon selection criteria, the $z_{0}$ 's of the muon candidates were required to be within $5 \mathrm{~cm}$ of each other and the invariant mass of the dimuon candidate was required to be between 81 and $101 \mathrm{GeV} / \mathrm{c}^{2}$. The invariant mass distributions of the $Z \rightarrow \mu \mu$ samples are shown in Figure 5.3. There were 344 events in Run $1 \mathrm{~A}$ and 1635 events in Run 1B.

The observed underlying event differs from the true underlying event, which is equal in magnitude and opposite in direction to the transverse momentum of the boson, due to the energy resolution of the calorimeters. Due to the correlation between $\vec{u}$ and the $p_{T}$ of the boson, the net energy flow in the observed underlying event was decomposed into the component parallel to the true underlying event, $u_{1}$, and the component perpendicular to the true underlying event, $u_{2}$, as shown in Figure 5.4. The $u_{1}$ and $u_{2}$ distributions as functions of the $Z$ boson $p_{T}$ for Run $1 \mathrm{~A}$ and Run $1 \mathrm{~B}$ are shown in Figures 5.5 and 5.6, respectively. In order to model $u_{L}$ and $u_{T}$ correctly, 

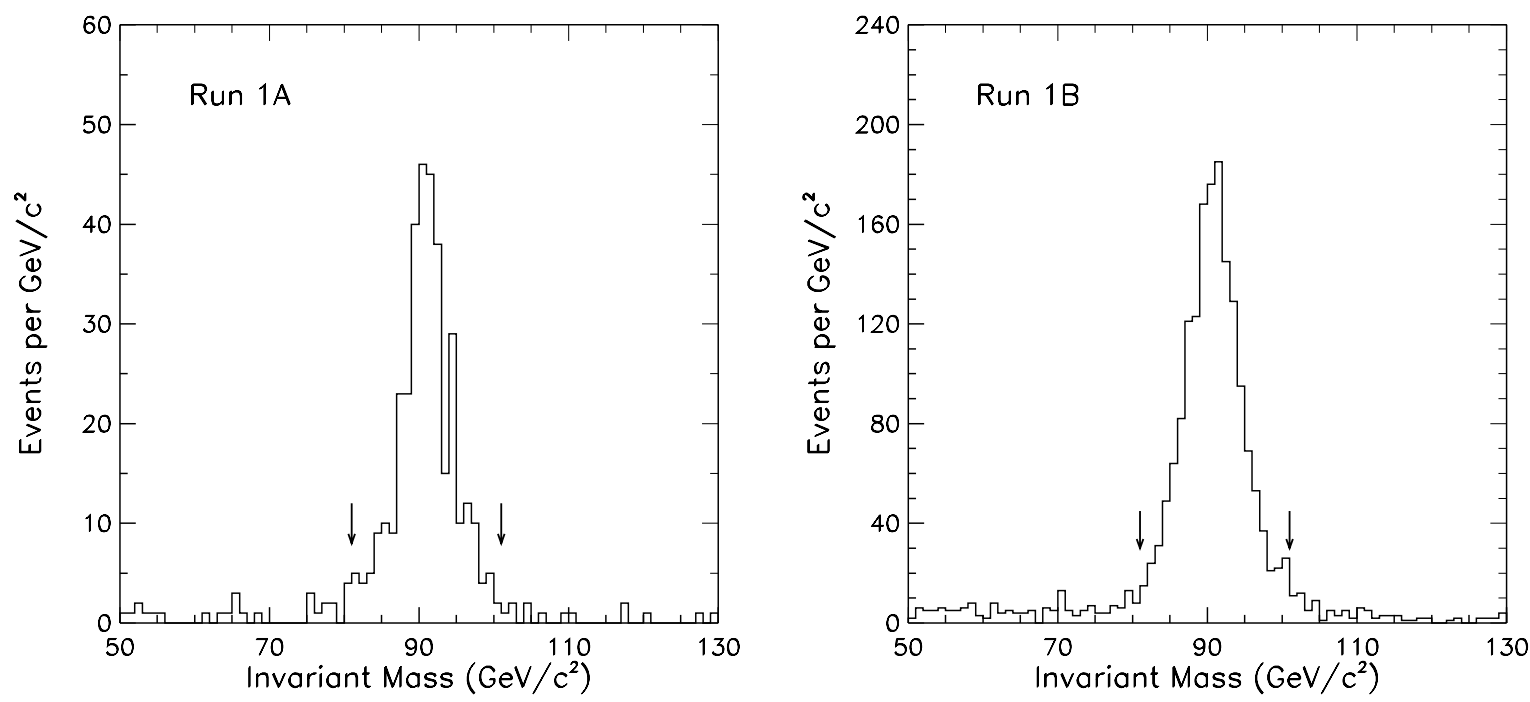

Figure 5.3: The invariant mass distributions of the $Z \rightarrow \mu \mu$ samples in Run $1 \mathrm{~A}$ (left) and Run $1 \mathrm{~B}$ (right). The events between the arrows $\left(81<M<101 \mathrm{GeV} / c^{2}\right)$ were used in the underlying event modelling. There are 344 events and 1635 events within the mass window in Run $1 \mathrm{~A}$ and Run $1 \mathrm{~B}$, respectively.

the observed $u_{1}$ and $u_{2}$ distributions and their correlation with the boson $p_{T}$ were first determined. These were then incorporated into the underlying event model to produce the correct correlations in the $u_{L}$ and $u_{T}$ variables.

The $u_{1}$ and $u_{2}$ distributions for a given $Z$ boson $p_{T}$ were parametrised with double Gaussian distributions. The probability density for the $i$ th event was assumed to have the form

$$
\begin{aligned}
p_{i}= & \left(\frac{1-R_{1}}{\sigma_{1}} \exp \left(-\frac{\left(u_{1_{i}}-\bar{u}_{1}\right)^{2}}{2 \sigma_{1}{ }^{2}}\right)+\frac{R_{1}}{\sigma_{2}} \exp \left(-\frac{\left(u_{1_{i}}-\bar{u}_{1}\right)^{2}}{2 \sigma_{2}{ }^{2}}\right)\right) \\
& \times\left(\frac{1-R_{2}}{\sigma_{3}} \exp \left(-\frac{u_{2_{i}}{ }^{2}}{2 \sigma_{3}{ }^{2}}\right)+\frac{R_{2}}{\sigma_{4}} \exp \left(-\frac{u_{2_{i}}{ }^{2}}{2 \sigma_{4}{ }^{2}}\right)\right),
\end{aligned}
$$

where

$$
\bar{u}_{1}=\left\{\begin{array}{cc}
m_{2} p_{T_{i}} & \left(p_{T_{i}} \leq m_{1}\right) \\
\left(m_{2}-m_{3}\right) m_{1}+m_{3} p_{T_{i}} & \left(p_{T_{i}}>m_{1}\right)
\end{array}\right.
$$




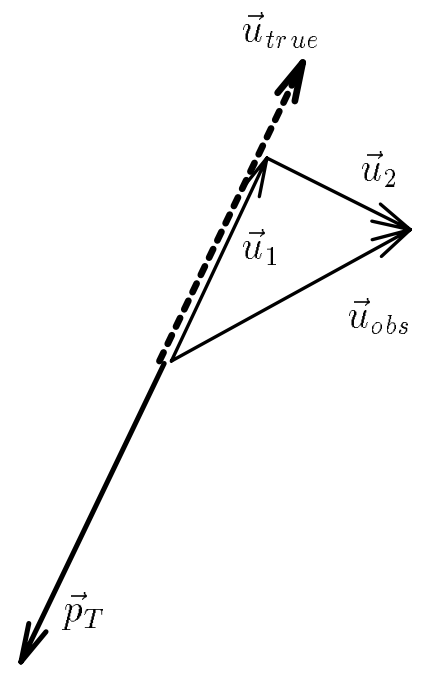

Figure 5.4: Decomposition of the observed underlying event, $\vec{u}_{\text {obs }}$, into components parallel, $\vec{u}_{1}$, and perpendicular, $\vec{u}_{2}$, to the true underlying event, $\vec{u}_{\text {true }}$. The energy flow $\vec{u}_{\text {true }}$ is equal in magnitude and opposite in direction to the transverse momentum vector of the boson $\vec{p}_{T}$.

and

$$
\begin{gathered}
\sigma_{1}=s_{1}+s_{2} p_{T_{i}} \\
\sigma_{3}=s_{3}+s_{4} p_{T_{i}} \\
\sigma_{2}=a_{1} \sigma_{1} \\
\sigma_{4}=a_{2} \sigma_{3} .
\end{gathered}
$$

The parameters $m_{i}, s_{j}, a_{k}$, and $R_{l}$ are determined by a likelihood fit of this probability density to the $Z \rightarrow \mu \mu$ sample. In this fit, $\bar{u}_{1}$ was forced to be zero at $p_{T}=0$ and $\bar{u}_{2}$ was assumed to be zero everywhere. ${ }^{1}$ The likelihood fit was performed with the MINUIT minimisation programme [52]. The results of the fit, presented in Table 5.1, show that the Run 1B underlying event has wider distributions around mean values of $u_{1}$ and $u_{2}$ than the underlying event in Run $1 \mathrm{~A}$. This was expected due to the larger mean number of collisions per beam bunch crossing in Run $1 \mathrm{~B}$ compared to Run $1 \mathrm{~A}$.

\footnotetext{
${ }^{1}$ Even if $\bar{u}_{1}$ was let float in the fit, the result was consistent with $\bar{u}_{1}=0$ at $p_{T}=0$ within the fitted uncertainty.
} 

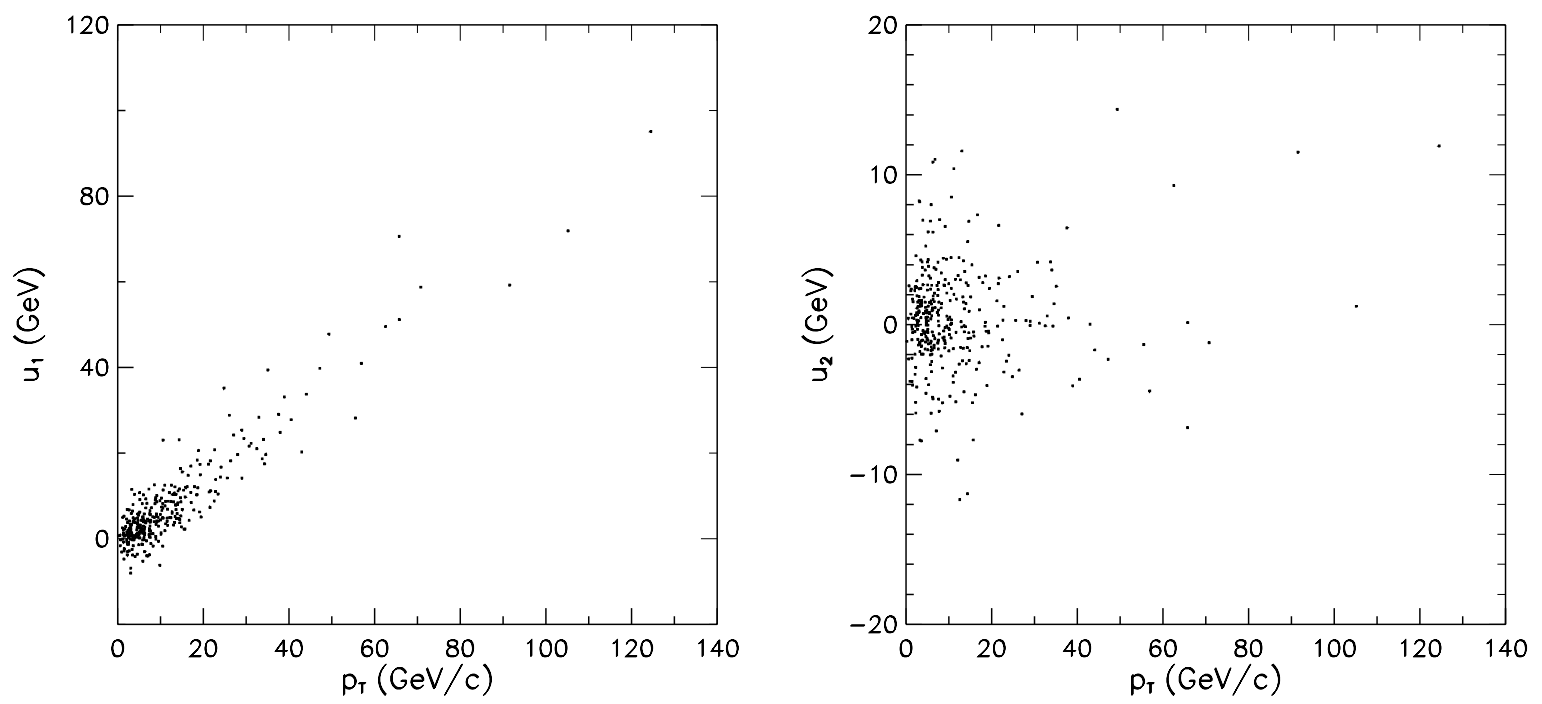

Figure 5.5: The $u_{1}$ vs $p_{T}$ (left) and $u_{2}$ vs $p_{T}$ (right) distributions of the $Z \rightarrow \mu \mu$ sample in Run $1 \mathrm{~A}$.
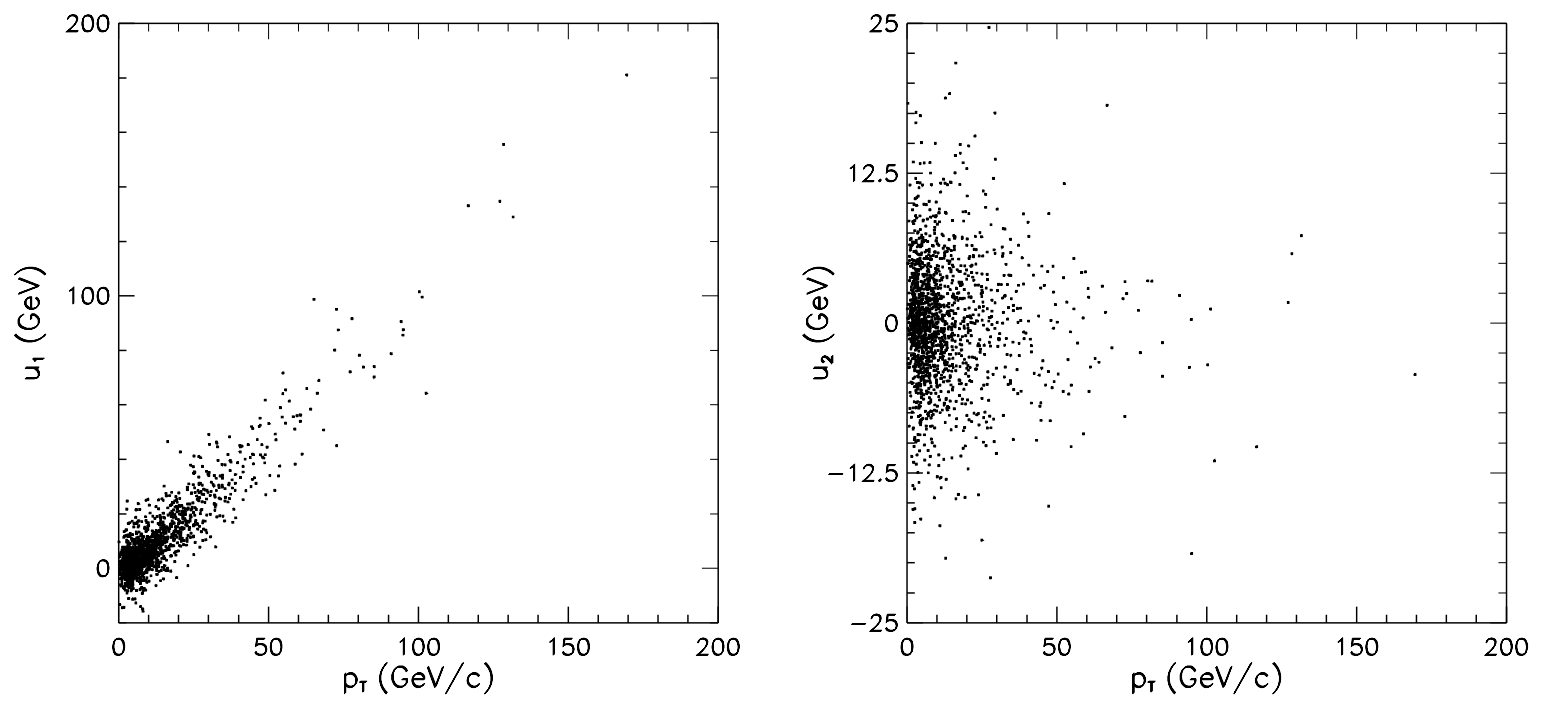

Figure 5.6: The $u_{1}$ vs $p_{T}$ (left) and $u_{2}$ vs $p_{T}$ (right) distributions of the $Z \rightarrow \mu \mu$ sample in Run 1B. 


\begin{tabular}{|c|cc|}
\hline Fit Parameters & Run 1A & Run 1B \\
\hline \hline$m_{1}$ & $7.74 \pm 0.90$ & $8.23 \pm 0.48$ \\
$m_{2}$ & $(4.49 \pm 0.31) \times 10^{-1}$ & $(4.97 \pm 0.21) \times 10^{-1}$ \\
$m_{3}$ & $(7.80 \pm 0.28) \times 10^{-1}$ & $1.01 \pm 0.02$ \\
$s_{1}$ & $1.80 \pm 0.27$ & $2.76 \pm 0.13$ \\
$s_{2}$ & $(9.02 \pm 1.64) \times 10^{-2}$ & $(9.13 \pm 0.93) \times 10^{-2}$ \\
$s_{3}$ & $1.60 \pm 0.16$ & $3.12 \pm 0.20$ \\
$s_{4}$ & $(3.31 \pm 0.96) \times 10^{-2}$ & $(2.14 \pm 0.46) \times 10^{-2}$ \\
$R_{1}$ & $(3.51 \pm 0.14) \times 10^{-1}$ & $(3.72 \pm 0.54) \times 10^{-1}$ \\
$R_{2}$ & $(3.78 \pm 0.79) \times 10^{-1}$ & $(4.15 \pm 0.62) \times 10^{-1}$ \\
$a_{1}$ & $2.04 \pm 0.17$ & $2.19 \pm 0.03$ \\
$a_{2}$ & $2.47 \pm 0.19$ & $2.06 \pm 0.08$ \\
\hline
\end{tabular}

Table 5.1: Results of the MINUIT fit to the $u_{1}$ and $u_{2}$ distributions for the $Z \rightarrow \mu \mu$ samples. See equations 5.3 to 5.5 for the definitions of the parameters. The $p_{T}$ is measured in units of $\mathrm{GeV} / c$.

Since the boson $p_{T}$ in the underlying event probability density function was a measured boson $p_{T}$ as opposed to the true boson $p_{T}$, the input $p_{T}$ to the probability density function must take into account the smearing due to detector resolution. For each Monte Carlo $W$ or $W^{\prime}$ boson generated as described in Section 5.1, a $Z \rightarrow \mu \mu$ event with the same boson $p_{T}$ as that of the $W$ or $W^{\prime}$ boson was also generated. The input $p_{T}$ of the $W$ or $W^{\prime}$ boson to the underlying event probability density function was replaced by the $Z$ boson $p_{T}$ reconstructed from the momenta of the muons with the detector model described in the following section. The underlying event model was then adjusted by "bootstrapping" to produce the fit results in Table 5.1.

In order to verify the accuracy of the resulting model, the $u_{L}$ and $u_{T}$ distributions of a Monte Carlo generated $Z \rightarrow \mu \mu$ sample that incorporated the underlying event model and of the $Z \rightarrow \mu \mu$ sample in Run 1B are compared in Figure 5.7. Also, $u_{L}$ and $u_{T}$ distributions of a Monte Carlo $W \rightarrow \mu \nu$ sample and of the $W / W^{\prime} \rightarrow \mu \nu$ event candidates in Run 1B are shown in Figure 5.8. The model and data distributions are in good agreement, validating the accuracy of this modelling technique. 

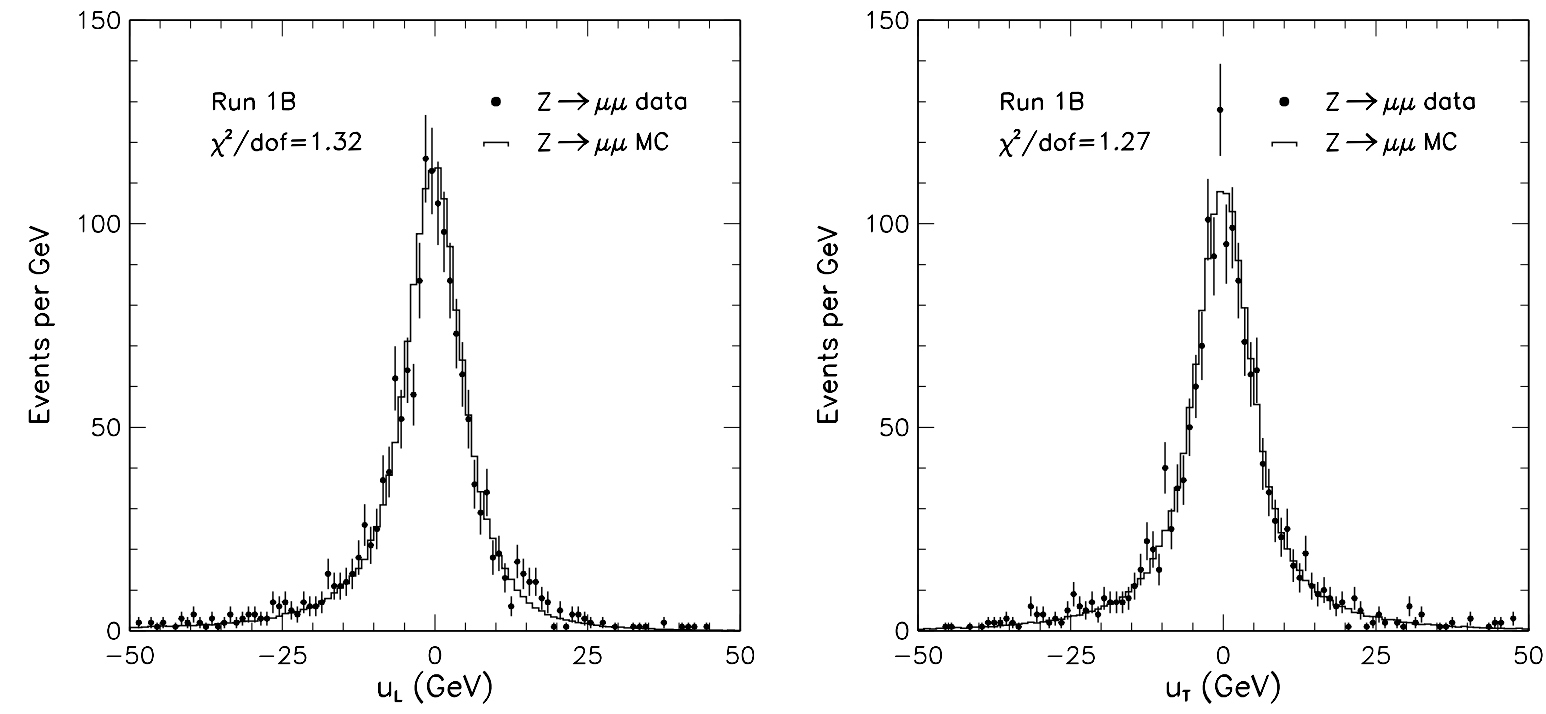

Figure 5.7: Comparisons between the $u_{L}$ distributions of the $Z \rightarrow \mu \mu$ data sample and a $Z \rightarrow \mu \mu$ Monte Carlo sample (left) and the $u_{T}$ distributions of the same samples (right) for Run 1B. The Monte Carlo distributions are normalised to the number of events in the $Z \rightarrow \mu \mu$ data sample within $\left|u_{L, T}\right|<50 \mathrm{GeV}$.
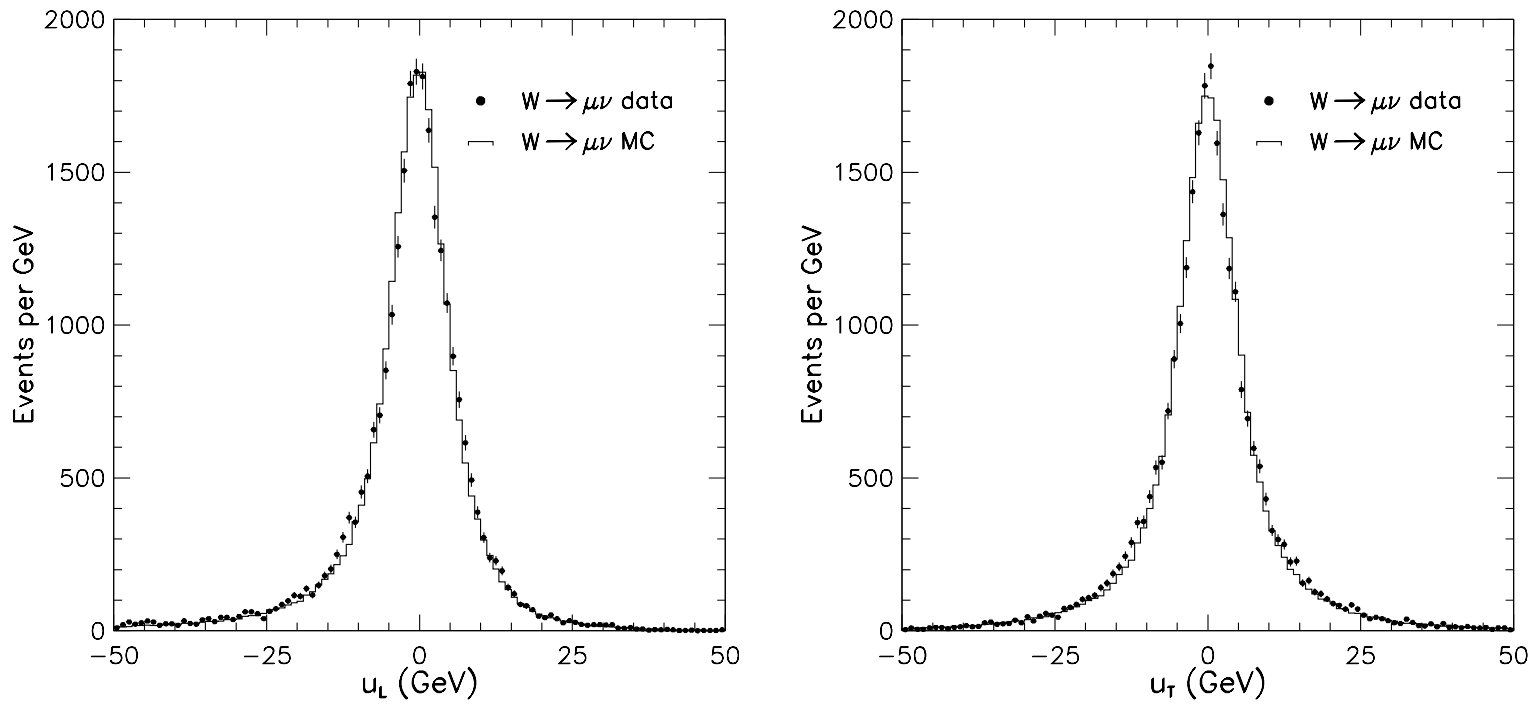

Figure 5.8: Comparisons between the $u_{L}$ distributions of the $W / W^{\prime} \rightarrow \mu \nu$ event candidate sample and a $W \rightarrow \mu \nu$ Monte Carlo sample (left) and the $u_{T}$ distributions of the same samples (right) for Run 1B. The Monte Carlo distributions were normalised to the number of events in the $W / W^{\prime} \rightarrow \mu \nu$ event candidate sample within $\left|u_{L, T}\right|<$ $50 \mathrm{GeV}$. 


\subsubsection{Detector Model}

The measured momentum of a muon is smeared due to the finite momentum resolution of the tracking system. The momentum resolution of a beam constrained fit of a track was measured to be [51]

$$
\delta p_{T} / p_{T}=\left\{\begin{array}{l}
(0.81 \pm 0.09) \times 10^{-3} p_{T}(\text { Run 1A }) \\
(1.01 \pm 0.06) \times 10^{-3} p_{T}(\text { Run 1B })
\end{array}\right.
$$

where $p_{T}$ is in units of $\mathrm{GeV} / c$. Since the transverse momentum was determined by the track's curvature, which is proportional to $1 / p_{T}$ and has a Gaussian resolution function, the curvature of the muon track was smeared with a Gaussian probability density function with widths of $0.81 \times 10^{-3}$ and $1.01 \times 10^{-3}$ for Run $1 \mathrm{~A}$ and Run $1 \mathrm{~B}$, respectively.

The distribution of the primary vertex along the $z$ direction was parametrised with a double Gaussian function fitted to the $z$ distribution of the class 12 vertices (see Section 3.4) in the events in Run 1B before applying any event selection cuts. The fitted double Gaussian function had means at $1.7 \mathrm{~cm}$ and $2.3 \mathrm{~cm}$ and widths of $33.9 \mathrm{~cm}$ and $18.4 \mathrm{~cm}$, respectively, in the detector model. The ratio of the areas of two Gaussians was approximately 2:1.

The efficiencies of the Level 1 and Level 2 triggers were taken into account using a model of their performance. The trigger simulation used the measured efficiencies and prescalings of the Level 1 and Level 2 triggers for Run 1B (see Section 4.1). The Run 1B trigger efficiencies were used for the CMUP and CMUNP Run 1A triggers, but with no prescaling for the CMUNP trigger. The parametrisations of the trigger efficiencies, with the $\eta$ dependence of the CFT efficiency incorporated, were used to determine the probability that a given Monte Carlo event satisfied the trigger requirements. The event selection efficiencies (see Chapter 4) were also parametrised to determine the probability of a given Monte Carlo event satisfying the event selection. The Level 3 triggers were not simulated but the effect of the $E_{\text {had }}<6 \mathrm{GeV}$ cut was accounted for in the event selection efficiency parametrisation.

The efficiency times acceptance for $W / W^{\prime} \rightarrow \mu \nu$ events for Run $1 \mathrm{~B}$ as a function 


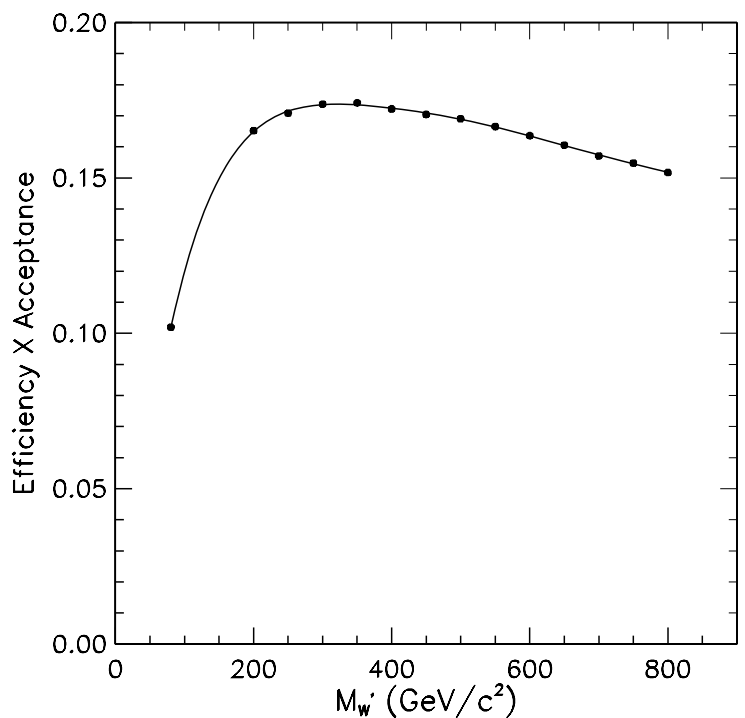

Figure 5.9: Efficiency times acceptance of $W / W^{\prime} \rightarrow \mu \nu$ events in Run $1 \mathrm{~B}$.

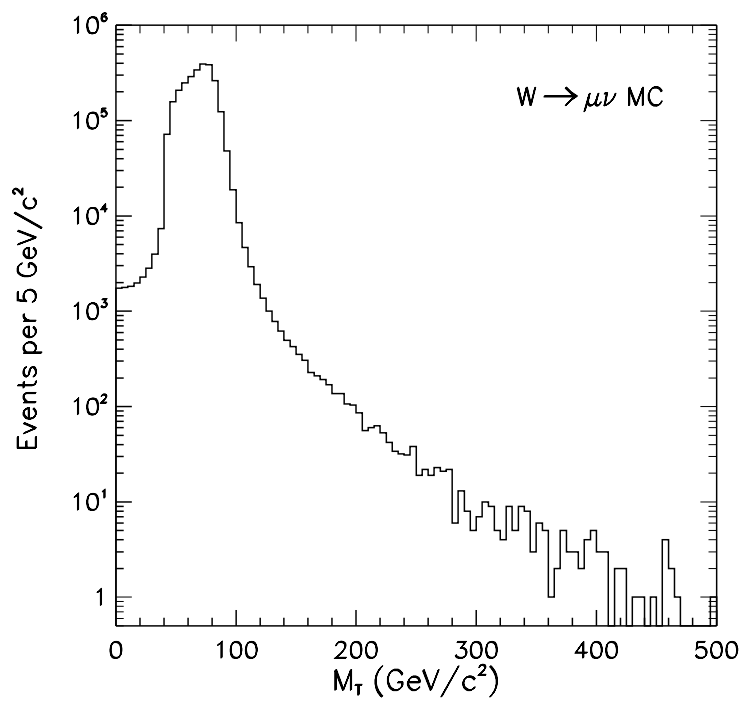

Figure 5.10: The expected transverse mass distribution of the observed $W \rightarrow \mu \nu$ events in Run 1B. The distribution shown is not normalised. 
of $W^{\prime}$ boson mass is shown in Figure 5.9. It rises as the $W^{\prime}$ boson mass increases in the low mass region due to an increase in the geometrical acceptance but falls in the high mass region due to the effects of cuts with muon momentum-dependent efficiencies (see Section 4.4).

The expected transverse mass distributions of observed $W \rightarrow \mu \nu$ and $W^{\prime} \rightarrow \mu \nu$ events are shown in Figures 5.10 and 5.11, respectively. The pile-up in the low transverse mass region for high mass $W^{\prime}$ bosons is due to the enhancement in the contribution from off-mass-shell $W^{\prime}$ bosons caused by the steeply falling proton and antiproton parton densities for partons with large momentum fractions. The position of the peak in the measured transverse mass distribution for a relatively heavy $W^{\prime}$ boson $\left(\gtrsim 600 \mathrm{GeV} / \mathrm{c}^{2}\right)$ is noticeably lower than the nominal mass due to the asymmetric resolution for very high- $p_{T}$ tracks. The expected transverse mass distributions of observed $W^{\prime} \rightarrow \mu \nu$ events overlaid with that of $W \rightarrow \mu \nu$ events are shown in Figure 5.12. 

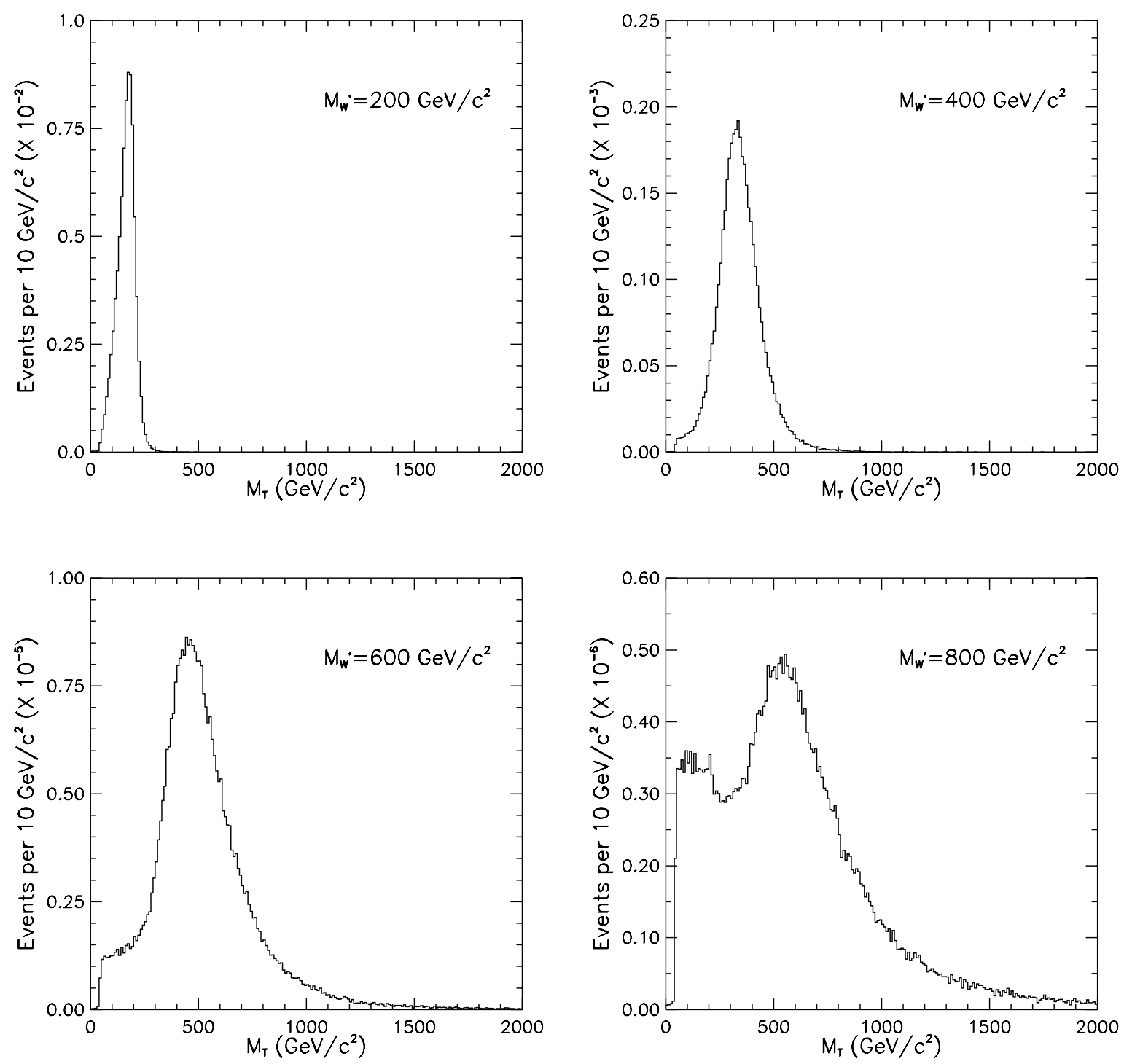

Figure 5.11: The expected transverse mass distributions of observed $W^{\prime} \rightarrow \mu \nu$ events for different $W^{\prime}$ boson masses for Run 1B. The distributions are shown in units of the expected number of observed $W \rightarrow \mu \nu$ events. The pile up in the low transverse mass region at high $M_{W^{\prime}}$ is due to contributions from off-mass-shell $W^{\prime}$ bosons (see text). 


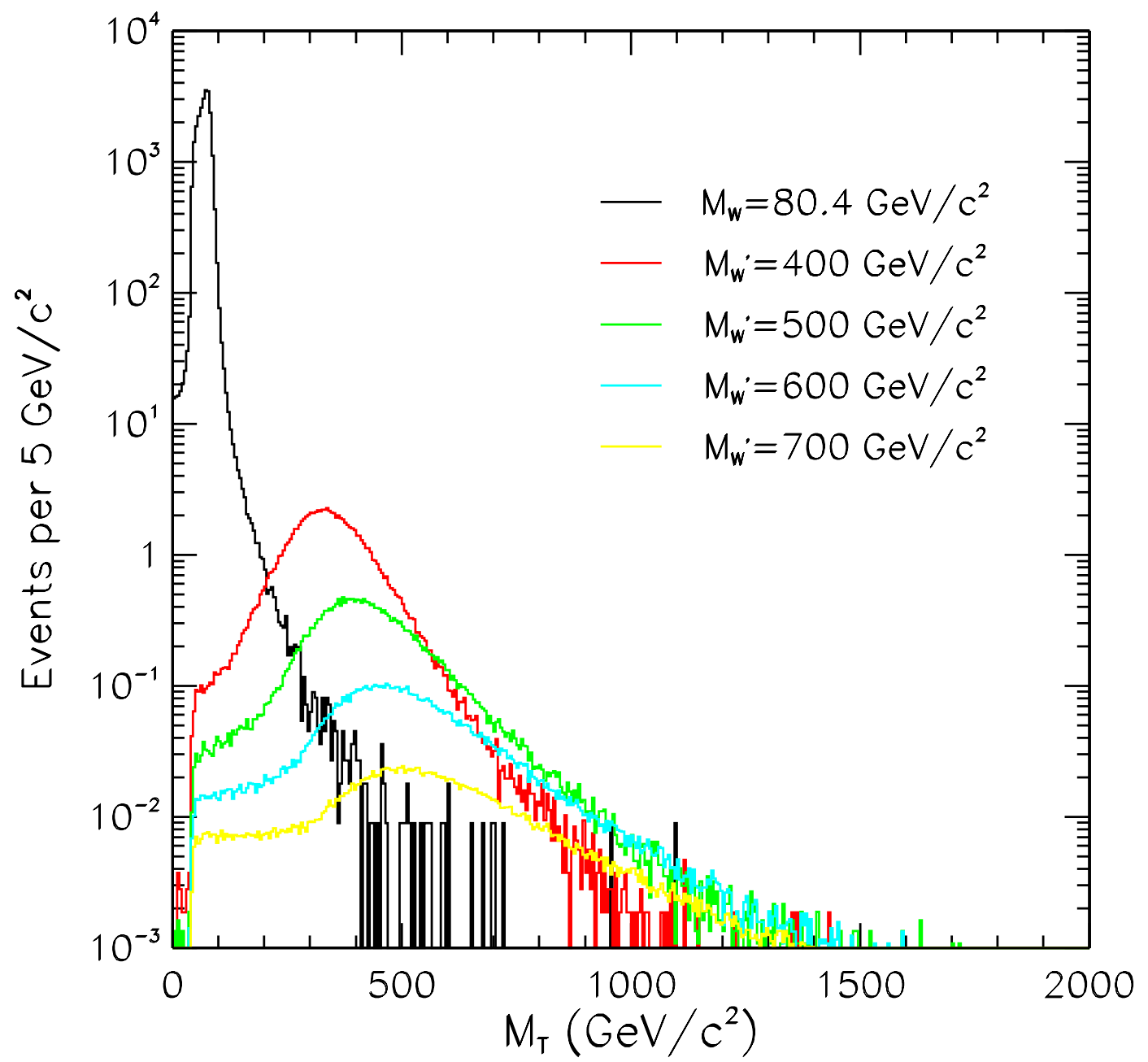

Figure 5.12: The expected transverse mass distributions of observed $W^{\prime} \rightarrow \mu \nu$ events for various masses overlaid with that of $W \rightarrow \mu \nu$ events for Run $1 \mathrm{~B}$. 


\section{Chapter 6}

\section{Backgrounds to $W^{\prime}$ Production}

Backgrounds to $W^{\prime} \rightarrow \mu \nu$ production come from sources with real muons such as $W \rightarrow \mu \nu, W \rightarrow \tau \nu \rightarrow \mu X, Z / \gamma \rightarrow \mu \mu, Z / \gamma \rightarrow \tau \tau \rightarrow \mu X, t \bar{t} \rightarrow \mu X$, and cosmic rays, and from QCD jet production that result in fake muon candidates. In this chapter, the background sources to $W^{\prime} \rightarrow \mu \nu$ production other than the $W \rightarrow \mu \nu$ process are described.

\section{1 $Z / \gamma \rightarrow \mu \mu$}

The $Z / \gamma \rightarrow \mu \mu$ process becomes a source of background when one of the muons is not identified as a muon candidate and the event then satisfies the $\mathbb{I}_{T}$ requirement. There are two cases: 1) The secondary muon falls outside the muon chamber coverage or 2) the secondary muon fails the secondary muon selection requirements for the $Z / \gamma \rightarrow \mu \mu$ rejection (see Section 3.6) and the event passes the "back-to-back" cuts (see Section 3.7).

Although it is possible to use a $Z \rightarrow \mu \mu$ event sample to construct a transverse mass distribution of the $Z / \gamma \rightarrow \mu \mu$ background by assuming one of the muons to be "lost", Monte Carlo calculations were used to determine this transverse mass distribution mainly due to statistical considerations. A sample of $Z / \gamma \rightarrow \mu \mu$ events was generated with the PYTHIA Monte Carlo programme using the CTEQ4A1 parton distribution function set. The $Z$ boson mass was set to $91.2 \mathrm{GeV} / c^{2}[53]$ and the minimum dimuon invariant mass of the $Z / \gamma \rightarrow \mu \mu$ events was $30 \mathrm{GeV} / c^{2}$. The events 
were reconstructed with the simple detector model described in Section 5.2.

The expected transverse mass distribution of $Z / \gamma \rightarrow \mu \mu$ events was normalised with respect to that of $W \rightarrow \mu \nu$ production using the measured ratio of the production cross section times branching fractions of the processes $p \bar{p} \rightarrow W X \rightarrow e \nu X$ to $p \bar{p} \rightarrow$ $Z X \rightarrow e e X[54]$

$$
\frac{\sigma \cdot \mathcal{B}(W \rightarrow e \nu)}{\sigma \cdot \mathcal{B}(Z \rightarrow e e)}=10.90 \pm 0.43
$$

assuming that $\frac{\sigma \cdot \mathcal{B}(W \rightarrow \mu \nu)}{\sigma \cdot \mathcal{B}(W \rightarrow e \nu)}=\frac{\sigma \cdot \mathcal{B}(Z \rightarrow \mu \mu)}{\sigma \cdot \mathcal{B}(Z \rightarrow e e)}=1$. The ratio of the cross sections for DrellYan $(Z / \gamma)$ to $Z$ boson production was used to correct for the non-resonant part of the $Z / \gamma$ production process:

$$
\int_{30 \mathrm{GeV} / c^{2}}^{\infty} \frac{d \sigma}{d M}(Z / \gamma) d M / \int_{30 \mathrm{GeV} / c^{2}}^{\infty} \frac{d \sigma}{d M}(Z) d M=1.25 \pm 0.01
$$

where $M$ is the invariant mass of the produced $Z$ or $Z / \gamma$ system. The expected $Z / \gamma \rightarrow \mu \mu$ event transverse mass distribution is shown in Figure 6.1. The relative expected rate of observed $Z / \gamma \rightarrow \mu \mu$ events with respect to the $W \rightarrow \mu \nu$ events is $(1.01 \pm 0.04) \times 10^{-1}$ for Run $1 B .^{1}$

\section{2 $W \rightarrow \tau \nu$ and $Z / \gamma \rightarrow \tau \tau$}

Backgrounds from $W$ and $Z$ production involving tau lepton decays have lower acceptances than the direct decays $W \rightarrow \mu \nu$ or $Z / \gamma \rightarrow \mu \mu$ since the additional neutrinos from the tau lepton decay carry off a fraction of the transverse momentum of the tau lepton. The expected contribution from the $W \rightarrow \tau \nu \rightarrow \mu \nu \nu \nu$ sequential decay process was modelled by a PYTHIA Monte Carlo calculation using the CTEQ4A1 parton distribution function set and the simple detector model. The resulting transverse mass distribution was normalised with respect to the $W \rightarrow \mu \nu$ process using the branching fraction $\mathcal{B}(\tau \rightarrow \mu \nu \nu)=0.1735 \pm 0.0010[55]$.

The $Z / \gamma \rightarrow \tau \tau \rightarrow \mu X$ decays are complicated by the presence of a second tau lepton, which can decay either leptonically or hadronically. The PYTHIA Monte

\footnotetext{
${ }^{1}$ The relative expected rate of observed $Z / \gamma \rightarrow \mu \mu$ events with respect to the $W \rightarrow \mu \nu$ events in Run $1 \mathrm{~A}$ is similar to that in Run $1 \mathrm{~B}$.
} 
Carlo programme was used to generate the sequential decay $Z / \gamma \rightarrow \tau \tau \rightarrow \mu X$ and the detector response was determined with the CDFSIM. The expected transverse mass distribution of $Z / \gamma \rightarrow \tau \tau$ events was normalised with respect to that of $W \rightarrow \mu \nu$ production in the same manner as that of $Z / \gamma \rightarrow \mu \mu$ production, taking into account the tau lepton branching fraction to $\mu \nu \nu \nu$. The expected transverse mass distributions for $W \rightarrow \tau \nu$ and $Z / \gamma \rightarrow \tau \tau$ events are shown in Figure 6.1 . The relative expected rates of the observed $W \rightarrow \tau \nu$ events and $Z / \gamma \rightarrow \tau \tau$ events with respect to the $W \rightarrow \mu \nu$ events are $(2.64 \pm 0.02) \times 10^{-2}$ and $(2.21 \pm 0.09) \times 10^{-3}$, respectively, for Run 1B.

\section{3 $t \bar{t}$ Production}

Top quark $(t \bar{t})$ production is not a large background since the cross section for this process is relatively small. However, because the top quark is heavy, the muons produced by a top quark decay are expected to have high $p_{T}$, thus contributing events to the high transverse mass region. There are two sources of muons from $t \bar{t}$ production: A top quark decays into a $b$-quark and $W$ boson pair and the $W$ boson decays into a $\mu \nu$ pair, or the $b$-quark decays into a muon and other fragments. Because the muon produced from $b$-quark decay is typically accompanied by hadrons and therefore is likely to fail the isolation cut imposed in the event selection, the muon from the $W$ boson decay is expected to be the main source of the $t \bar{t}$ production.

A sample of $t \bar{t}$ events were generated with the PYTHIA Monte Carlo programme using the MRS-R2 parton distribution function set [56]. The mass of top quark was set to be $176 \mathrm{GeV} / c^{2}[17]$. The detector response was modelled with CDFSIM. The resulting transverse mass distribution was normalised by the measured $t \bar{t}$ production cross section of $7.5_{-1.6}^{+1.9} \mathrm{pb}[57]$ and is shown in Figure 6.1 The relative expected rate of the observed $t \bar{t}$ production events with respect to the $W \rightarrow \mu \nu$ events is $\left(5.99_{-1.20}^{+1.52}\right) \times 10^{-4}$ for Run $1 \mathrm{~B}$. 

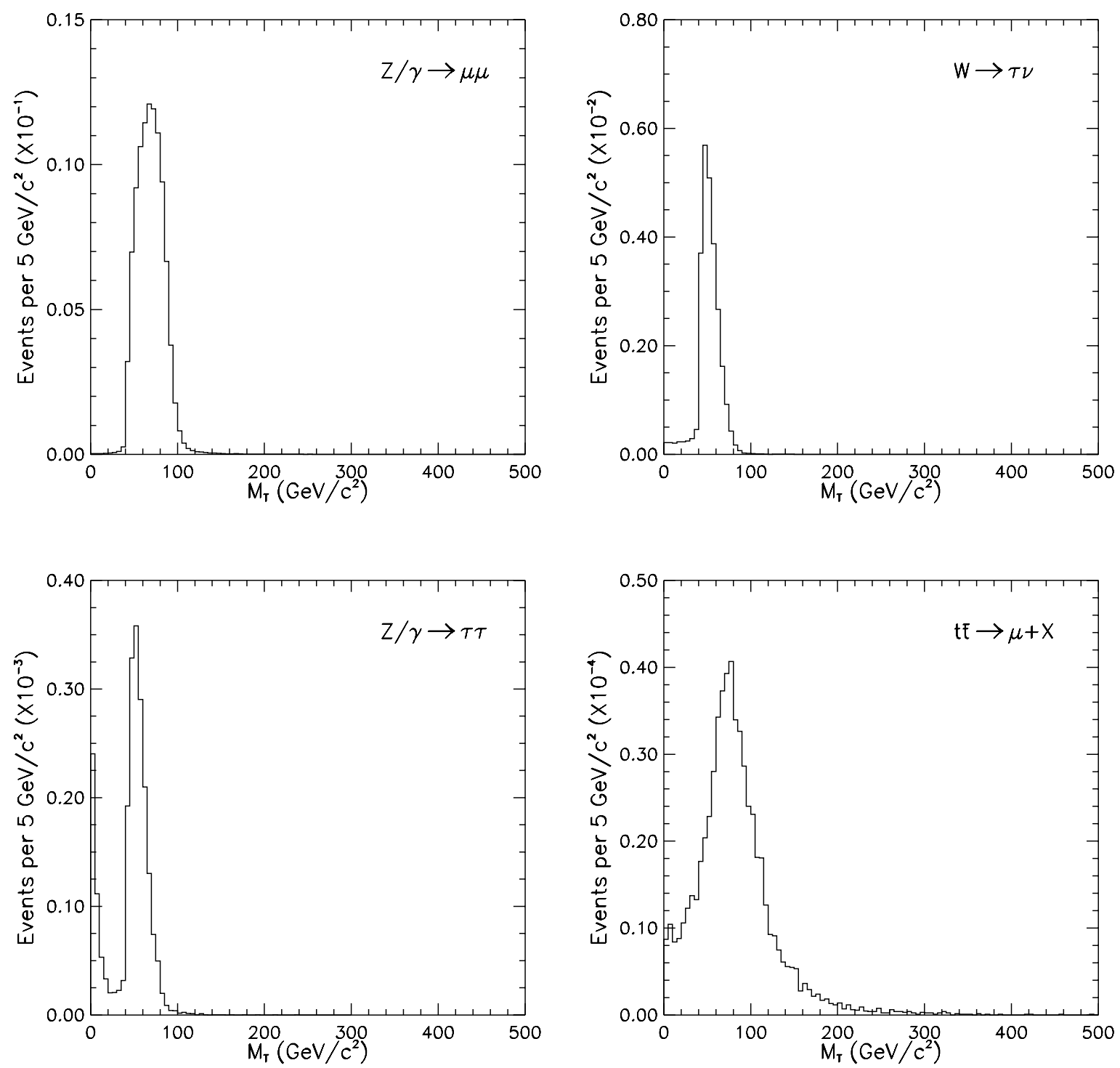

Figure 6.1: Expected transverse mass distributions of $Z / \gamma \rightarrow \mu \mu, W \rightarrow \tau \nu, Z / \gamma \rightarrow$ $\tau \tau$, and $t \bar{t}$ production normalised with respect to the expected number of observed $W \rightarrow \mu \nu$ events Run 1B. The distributions are shown in units of expected number of observed $W \rightarrow \mu \nu$ events. 


\subsection{QCD Background}

Background to the $W / W^{\prime} \rightarrow \mu \nu$ events from high- $p_{T}$ light quark and gluon production (QCD background) is expected when one or more of the resulting jets are misidentified as isolated muons that, in turn, result in a significant mismeasurement of $\mathbb{F}_{T}$.

The contribution from QCD background was calculated using a modified $W / W^{\prime} \rightarrow$ $\mu \nu$ event candidate sample created by removing the isolation requirement on the primary muon candidate. Since QCD background has more than one jet in an event, events in this sample were required to have at least one jet with observed $E_{T}$ greater than $5 \mathrm{GeV}$ that was at least $30^{\circ}$ in azimuth away from the primary muon candidate. Three subsamples were created using different isolation requirements: Iso $<1 \mathrm{GeV}$ ("muon enriched"), Iso $<3 \mathrm{GeV}$ ("W+ jets"), and Iso $>6 \mathrm{GeV}$ ("dijet enriched"). The "muon enriched" sample was expected to mainly comprise events that have a $W$ boson produced with jets, whereas the "dijet enriched" sample was expected to have an enhanced component of QCD background. The " $W+$ jets" sample was simply a subset of the $W / W^{\prime} \rightarrow \mu \nu$ event candidate sample with an additional jet requirement and was expected to contain the majority of the QCD background in the candidate sample. Since a majority of QCD background events come from dijet topologies, the azimuthal opening angle between the primary muon candidate and the jet with the highest $E_{T}, \phi(\mu, j)$, would be expected to have a distribution that peaks at $180^{\circ}$ for QCD background whereas the distribution of $\phi(\mu, j)$ for the "muon enriched" sample would not display this effect as prominently.

In order to estimate the number of QCD background events in the " $W+$ jets" sample, the $\phi(\mu, j)$ distribution of the " $W+$ jets" sample was fitted to the sum of the "muon enriched" sample and the "dijet enriched" sample simultaneously, as shown in Figure 6.2, with the relative fraction of each component being the only free parameter. From the fit, the numbers of QCD background events in the $W / W^{\prime} \rightarrow \mu \nu$

event candidate sample were determined to be $37_{-37}^{+54}$ events for Run $1 \mathrm{~A}$ and $201 \pm 99$ events for Run 1B.

The QCD background transverse mass distribution was assumed to be well represented by that of the "dijet enriched" sample and was normalised to the expected 

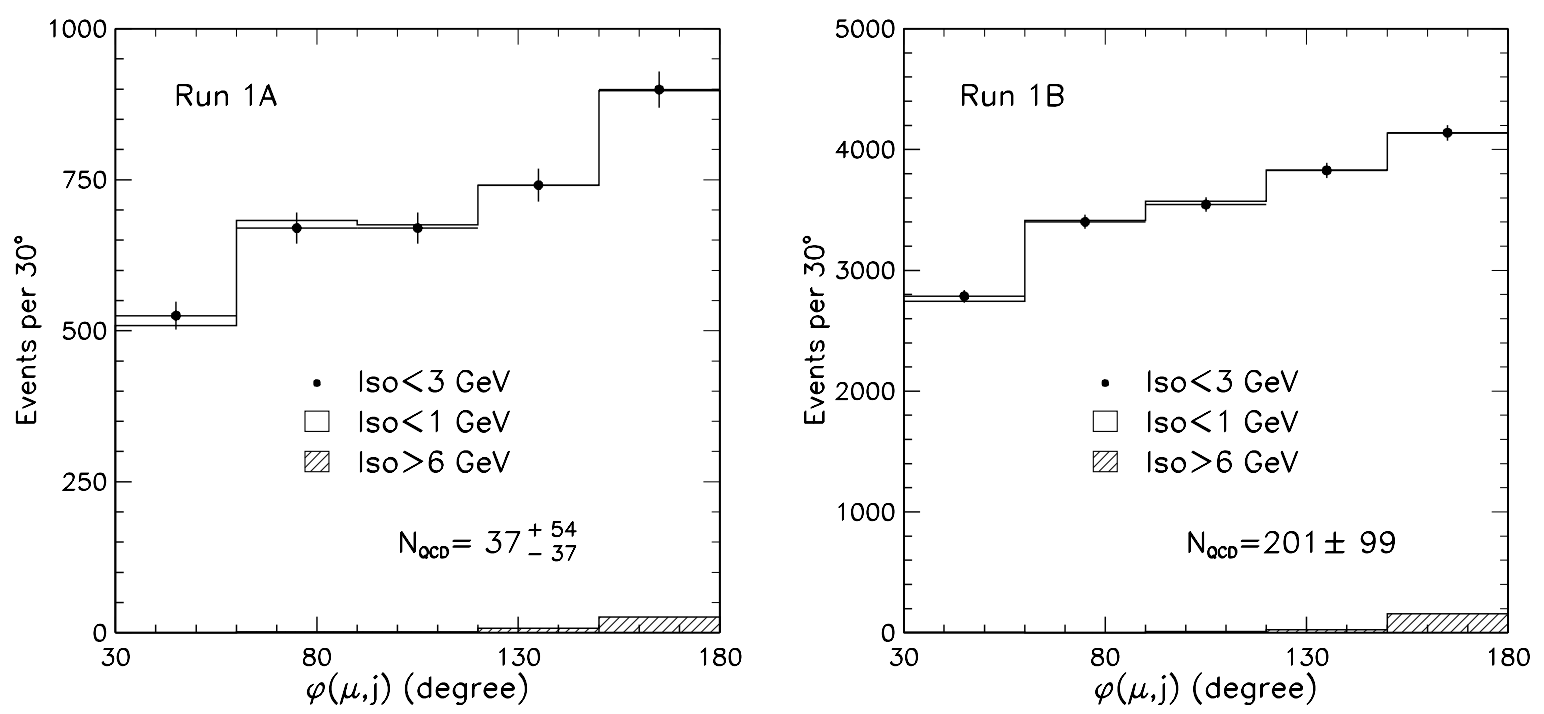

Figure 6.2: The distribution of the azimuthal opening angle between the muon candidate and the highest $E_{T}$ jet, $\phi(\mu, j)$, for the " $W+$ jets" sample $(I s o<3 \mathrm{GeV})$ fitted with the "muon enriched" sample $($ Iso $<1 \mathrm{GeV})$ and the "dijet enriched" sample (Iso $>6 \mathrm{GeV}$ ) for Run $1 \mathrm{~A}$ and Run $1 \mathrm{~B}$.

number of QCD background events. The transverse mass distribution of Run 1B "dijet enriched" sample was used to model the QCD background transverse mass distributions for both Run 1A and Run 1B because of the low statistics of the Run $1 \mathrm{~A}$ "dijet enriched" sample. The effect of this substitution was expected to be negligible due to the fact that the predicted number of QCD background in Run 1A was small. Figure 6.3 shows the predicted transverse mass distribution of QCD background for Run 1B.

\subsection{Cosmic Rays}

As was seen in Figure 3.7, the $W / W^{\prime} \rightarrow \mu \nu$ event candidates and the cosmic rays had different hadron TDC distributions. This was exploited to estimate the number of remnant cosmic ray events in the $W / W^{\prime} \rightarrow \mu \nu$ event candidate sample.

The hadron TDC distribution of the $W / W^{\prime} \rightarrow \mu \nu$ event candidate sample without the hadron TDC cut was fitted using a double Gaussian parametrisation in the region where the non-cosmic ray events dominate $\left(\left|t_{\text {had }}\right|<15 \mathrm{~ns}\right)$. The number of events in 


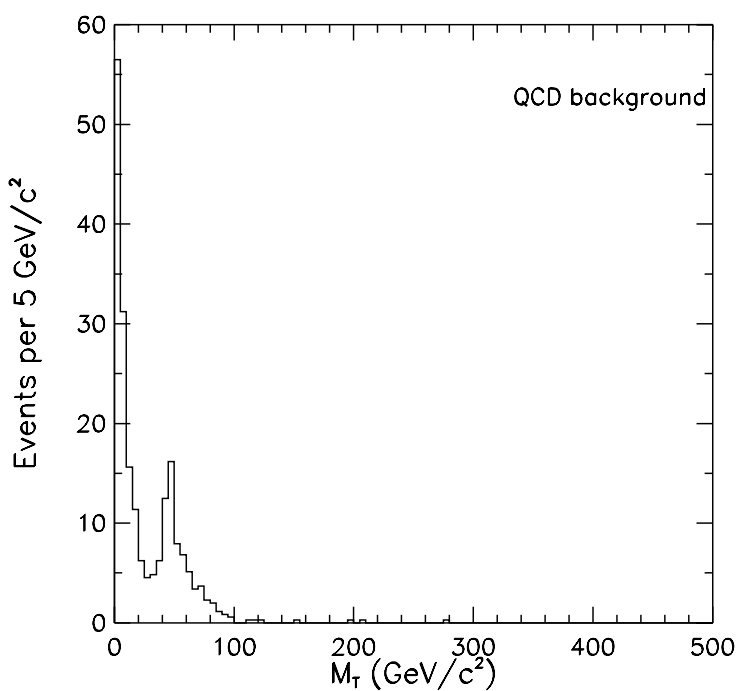

Figure 6.3: The transverse mass distribution of QCD background normalised to the expected number of events in Run 1B.

the $t_{\text {had }}<-7.8 \mathrm{~ns}$ region was subtracted from the number of events from the fit in the same region. The difference was the expected number of cosmic ray events outside the signal region of the hadron TDC distribution. The hadron TDC distribution of the cosmic ray sample (see Section 4.3) was then normalised to the number of expected cosmic ray events with $t_{\text {had }}<-7.8 \mathrm{~ns}$. This yielded $12_{-8}^{+31}$ cosmic ray events in the hadron TDC signal region.

Given that the estimated number of cosmic ray background events in the final sample was negligible compared to the total number of $W / W^{\prime} \rightarrow \mu \nu$ event candidates and these events are expected to have a low transverse mass distribution, the effect of the cosmic ray background will remain insignificant. Therefore this background contribution was ignored. ${ }^{2}$

\footnotetext{
${ }^{2}$ If all cosmic ray events were assumed to be in the low transverse mass region, where the $W \rightarrow \mu \nu$ background was expected to be dominant compared to the expected number of signal events, the decrease in the expected number of $W \rightarrow \mu \nu$ events was at most $\sim 0.2 \%$.
} 


\section{Chapter 7}

\section{Results}

As in any new particle search, the results of this study could either be an observation of the particle or quantitative limits on the rate for producing such an object. Since no clear signal for $W^{\prime} \rightarrow \mu \nu$ decay was observed, in this chapter the methodology of extracting limits from the data using the information about the expected backgrounds and possible signal is presented along with the search results.

\subsection{Expected Number of $W^{\prime} \rightarrow \mu \nu$ Events}

The expected number of observed $W^{\prime} \rightarrow \mu \nu$ events, $N_{W^{\prime}}$, is calculated as

$$
N_{W^{\prime}}=\frac{[\sigma \cdot \mathcal{B} \cdot \varepsilon \cdot A]_{W^{\prime}}}{[\sigma \cdot \mathcal{B} \cdot \varepsilon \cdot A]_{W}} \times N_{W},
$$

where $[\sigma \cdot \mathcal{B} \cdot \varepsilon \cdot A]_{W\left(W^{\prime}\right)}$ is the product of the production cross section, branching fraction, efficiency, and acceptance of $W \rightarrow \mu \nu\left(W^{\prime} \rightarrow \mu \nu\right)$ events. The quantity $N_{W}$ is the expected number of observed $W \rightarrow \mu \nu$ events in the $W / W^{\prime} \rightarrow \mu \nu$ event candidate sample, which was determined by the fit described in the following section. ${ }^{1}$ The expected numbers of observed $W^{\prime} \rightarrow \mu \nu$ events in the $W / W^{\prime} \rightarrow \mu \nu$ event candidates in Run $1 \mathrm{~A}+1 \mathrm{~B}$ are shown in Table 7.1 .

\footnotetext{
${ }^{1}$ In obtaining $N_{W}$, it was assumed that there was no $W^{\prime} \rightarrow \mu \nu$ events in the data sample.
} 


\begin{tabular}{|c|cc|}
\hline$M_{W^{\prime}}\left(\mathrm{GeV} / c^{2}\right)$ & $\frac{\sigma \cdot \mathcal{B}\left(W^{\prime} \rightarrow \mu \nu\right)}{\sigma \cdot \mathcal{B}(W \rightarrow \mu \nu)}$ & $N_{W^{\prime}}$ \\
\hline \hline $80.4(W)$ & 1.00 & 28200 \\
200 & $5.12 \times 10^{-2}$ & 2330 \\
250 & $2.09 \times 10^{-2}$ & 984 \\
300 & $9.50 \times 10^{-3}$ & 456 \\
350 & $4.66 \times 10^{-3}$ & 224 \\
400 & $2.40 \times 10^{-3}$ & 115 \\
450 & $1.28 \times 10^{-3}$ & 60.2 \\
500 & $6.95 \times 10^{-4}$ & 32.5 \\
550 & $3.83 \times 10^{-4}$ & 17.2 \\
600 & $2.14 \times 10^{-4}$ & 9.69 \\
650 & $1.21 \times 10^{-4}$ & 5.37 \\
700 & $6.93 \times 10^{-5}$ & 3.01 \\
750 & $4.03 \times 10^{-5}$ & 1.72 \\
800 & $2.40 \times 10^{-5}$ & 1.01 \\
\hline
\end{tabular}

Table 7.1: The ratios of cross sections times branching fractions of $W^{\prime} \rightarrow \mu \nu$ to $W \rightarrow \mu \nu$ productions and the expected numbers of observed $W^{\prime} \rightarrow \mu \nu$ events, $N_{W^{\prime}}$, in Run $1 \mathrm{~A}+1 \mathrm{~B}$. The expected numbers of observed events were obtained from the fits described in Sections 7.2. The number of events for $M_{W^{\prime}}=80.4 \mathrm{GeV} / c^{2}$ is the number of $W \rightarrow \mu \nu$ event candidates determined from the same fit. 


\subsection{Transverse Mass Distribution Fitting}

\subsubsection{Fitting Method}

To determine the observed number of $W^{\prime} \rightarrow \mu \nu$ events in the $W / W^{\prime} \rightarrow \mu \nu$ event candidate sample (or to set a limit on this yield), the transverse mass distribution of the candidate events was simultaneously fitted with the expected background distributions and a signal distribution using an unbinned likelihood fit method.

A likelihood, $L$, can be written as

$$
L=\frac{n_{e}^{n_{o}}}{n_{o} !} \exp \left(-n_{e}\right) \prod_{i=1}^{n_{o}}\left(\frac{\mathcal{P}_{i}}{n_{e}}\right)
$$

where $n_{0}$ is the observed number of events in the data, $n_{e}$ is the mean number of expected events, and $\mathcal{P}_{i}$ is the unnormalised probability for the $i$ th event to be produced [58]. The Poisson probability term, $\left(n_{e}{ }^{n_{0}} / n_{0} !\right) \exp \left(-n_{e}\right)$, constrains $n_{e}$ to be approximately equal to $n_{0}$. The "lineshape" term, $\Pi\left(\mathcal{P}_{i} / n_{e}\right)$, is what primarily constrains the unknown variables that are being determined by the fit. ${ }^{2}$

The quantity $\mathcal{P}_{i}$ is the probability of the $i$ th event having the observed transverse mass value. This is simply proportional to the size of the expected transverse mass distribution of the combination of background and signal events at the observed value of $M_{T}$. The unnormalised probability distribution at a given transverse mass value $M_{T}, \mathcal{P}\left(M_{T}\right)$, can be written as

$$
\mathcal{P}\left(M_{T}\right)=\alpha_{0}\left[W\left(M_{T}\right)+\sum_{j} \alpha_{j} B_{j}\left(M_{T}\right)+\beta W^{\prime}\left(M_{T}, M_{W^{\prime}}\right)\right]+\gamma Q\left(M_{T}\right)
$$

where $W\left(M_{T}\right)$ is the transverse mass lineshape of the $W \rightarrow \mu \nu$ process, $B_{j}\left(M_{T}\right)$ are the transverse mass lineshapes of the background other than the $W \rightarrow \mu \nu$ process and QCD background (i.e. $W \rightarrow \tau \nu, Z / \gamma \rightarrow \mu \mu, Z / \gamma \rightarrow \tau \tau$, and $t \bar{t}$ production), $W^{\prime}\left(M_{T}, M_{W^{\prime}}\right)$ is the transverse mass lineshapes of the $W^{\prime} \rightarrow \mu \nu$ process, and $Q\left(M_{T}\right)$ is the transverse mass distribution of QCD background. ${ }^{3}$ Each lineshape, excluding

\footnotetext{
${ }^{2}$ This maximum likelihood fit method is based on an a priori knowledge of the shape of the probability density distributions of the event distributions.

${ }^{3}$ For each event, each transverse mass lineshape was averaged over within $\pm 2.5 \mathrm{GeV} / \mathrm{c}^{2}$ of the
} 
QCD background, was normalised to its expected number of events relative to the $W \rightarrow \mu \nu$ process. The parameters $\alpha_{0}, \alpha_{j}(j \neq 0), \beta$, and $\gamma$ are the parameters to be determined by the fit.

The parameters $\alpha_{j}(j \neq 0)$ and $\beta$ are multiplied by the overall normalisation $\alpha_{0}$. Therefore, the fit estimates $\alpha_{j}(j \neq 0)$ and $\beta$ as fractional yields of non-QCD backgrounds (excluding the $W \rightarrow \mu \nu$ process) and the $W^{\prime} \rightarrow \mu \nu$ process, respectively, normalised with respect to that of the $W \rightarrow \mu \nu$ process. This removes a large fraction of the systematic uncertainties that come from cross section and efficiency calculations, and completely removes the systematic uncertainty that arises from the integrated luminosity of the event sample. Because QCD background was estimated from the data and, thus, is not affected by the aforementioned factors common to Monte Carlo generated events, $\gamma$ is in principle independent of other background sources and the signal. If there exists a $W^{\prime} \rightarrow \mu \nu$ process, one would expect to have $\beta=1$ when the the correct choice of $M_{W^{\prime}}$ is made for $W^{\prime}\left(M_{T}, M_{W^{\prime}}\right)$. The expected number of events from the fit, $n_{e}$, is calculated by

$$
n_{e}=\int \mathcal{P}\left(M_{T}\right) d M_{T}
$$

Since it is more convenient to deal with the negative log-likelihood function, from Eq. (7.2),

$$
-\ln L=n_{e}-n_{o} \ln n_{e}-\sum_{i} \ln \left(\frac{\mathcal{P}_{i}}{n_{e}}\right)+\text { constant }
$$

where the constant is not a function of the fit parameter and can be ignored. The minimisation of the negative log-likelihood was performed with the MINUIT minimisation programme [52].

\subsubsection{Statistical Fluctuation and Smoothing}

Any fluctuations due to low statistics in the high transverse mass region of the background transverse mass lineshapes need to be "smoothed out" for fitting. There are

$\overline{M_{T} \text { value of the event in calculating } \mathcal{P}}\left(M_{T}\right)$. Therefore, strictly speaking, $\mathcal{P}\left(M_{T}\right)$ is an averaged probability distribution for a given transverse mass value $M_{T}$. 
at least two ways to approach this issue; piecewise smoothing or parametrisation. The latter was used in this analysis since the piecewise smoothing tended to smear the distribution. The high transverse mass regions $\left(150<M_{T}<1000 \mathrm{GeV} / c^{2}\right)$ of the distributions for the Monte Carlo generated $W \rightarrow \mu \nu, Z / \gamma \rightarrow \mu \mu$, and $t \bar{t}$ production events were fitted with

$$
\frac{d n}{d M_{T}}=C /\left(M_{T}\right)^{P}
$$

where $C$ and $P$ were the parameters to be determined by a fit to each background component.

The low statistics in the high transverse mass region of the QCD background required that a different technique be used to smooth the background distribution. The fit region was extended down to $M_{T}>50 \mathrm{GeV} / c^{2}$ and a parametrisation that accounted for phase space with momentum smearing using an unbinned likelihood fitting technique was used:

$$
\frac{d n}{d M_{T}}=\int C\left(1-M_{T}^{\prime} / \sqrt{s}\right)^{N} /\left(M_{T}^{\prime}\right)^{P} \times S\left(M_{T}^{\prime} ; M_{T}\right) d M_{T}^{\prime}
$$

where $C, N$, and $P$ were parameters to be determined by the fit, $\sqrt{s}$ was the centerof-mass energy, $1.8 \mathrm{TeV}$, and $S\left(M_{T}^{\prime} ; M_{T}\right)$ was a smearing factor, which was defined as

$$
S\left(M_{T}^{\prime} ; M_{T}\right)=\frac{1}{\sqrt{2 \pi}\left(\delta p_{T} / p_{T}^{2}\right)} \times \exp \left(-\frac{\left(2 / M_{T}-2 / M_{T}^{\prime}\right)^{2}}{2\left(\delta p_{T} / p_{T}^{2}\right)^{2}}\right) .
$$

The smearing was used to account for the fact that $M_{T}$ was measured with the $p_{T}$ of a muon. Here, it was assumed that $M_{T}=2 p_{T}$. Figure 7.1 shows the transverse mass distributions of the $W \rightarrow \mu \nu, Z / \gamma \rightarrow \mu \mu, t \bar{t}$ production, and QCD backgrounds overlaid with their smooth parametrisations. The transverse mass distributions for the $W \rightarrow \tau \nu$ and $Z / \gamma \rightarrow \tau \tau$ processes were not parametrised because their contributions in the high transverse mass region were negligible.

\subsubsection{Fit Results}

When performing the fit, the fit parameters for backgrounds other than the $W \rightarrow \mu \nu$ process, $\alpha_{j}(j \neq 0)$ and $\gamma$, were fixed to unity. This was done to avoid instabilities 

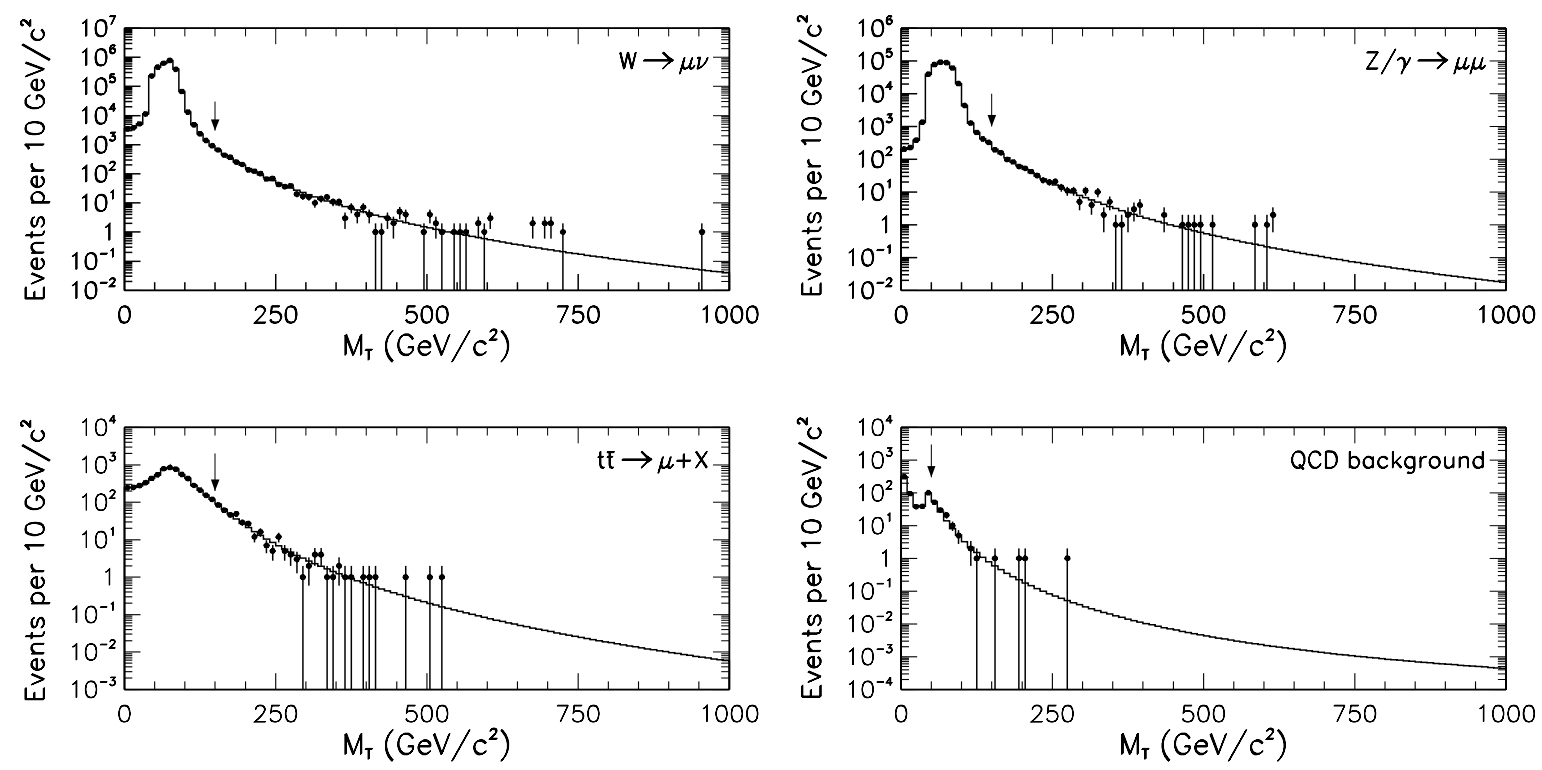

Figure 7.1: Parametrised transverse mass distributions of $W \rightarrow \mu \nu, Z / \gamma \rightarrow \mu \mu, t \bar{t}$ production, and QCD background for Run 1B. The arrows show the lower bound of the parametrised $M_{T}$ regions. The distributions shown here are not normalised.

in the fit due to several background sources having very similar transverse mass lineshapes. The effect of fixing the fit parameters was accounted for in the systematic uncertainty calculation described in Section 7.3. Also, $\alpha_{0}$ and $\beta$ were constrained to be in the physical region, i.e. $\alpha_{0}, \beta \geq 0$ [59]. The fit was performed in the region of $40<M_{T}<2000 \mathrm{GeV} / c^{2}$. This fit region reflects the difficulties in modelling the transverse mass lineshapes in the low and high transverse mass regions. The fraction of observed events expected above $M_{T}=2000 \mathrm{GeV} / c^{2}$ for an $M_{W^{\prime}}=800 \mathrm{GeV} / c^{2} W^{\prime}$ boson was estimated to be $\sim 2 \%$ and the impact of the loss of events on the results due to the the choice of fit region is small.

Two schemes were considered to extract the combined results from the two sets of data taken under slightly different conditions, i.e. Run $1 \mathrm{~A}$ and Run 1B. The likelihood distributions can be calculated for each data set and then combined to extract a common results. The other method is to combine the data sets, calculate the likelihood distribution for this single sample, and extract the common results. The latter was chosen for this analysis because the transverse mass distributions for the two samples were very similar. 
In order to fit the transverse mass distribution of the combined Run $1 \mathrm{~A}$ and Run 1B $W / W^{\prime} \rightarrow \mu \nu$ event candidates, the predicted transverse mass lineshapes configured for each data set were needed before they were added together. The transverse mass distribution of the $W / W^{\prime} \rightarrow \mu \nu$ event candidates from each data set was fitted separately with their respective background transverse mass lineshapes, as shown in Figure 7.2. The resulting $\alpha_{0}$ from each fit determines the scale factor for the transverse mass lineshapes for each data set. The ratio of $\alpha_{0}$ 's for Run 1A to Run 1B was $0.2214 \pm 0.0033$. This ratio accounts for the differences in the integrated luminosity and the efficiencies between Run 1A and Run 1B. The transverse mass lineshapes of backgrounds, except that of QCD background, for each data set were normalised by $\alpha_{0}$ from the separate for Run $1 \mathrm{~A}$ and Run $1 \mathrm{~B}$ fit. ${ }^{4}$ The normalised transverse mass lineshapes for each data set were added lineshape by lineshape to obtain the transverse mass lineshapes for the combined data set. This method avoids any systematic uncertainties arising from the relative integrated luminosity measurements as well as any absolute efficiency measurements when combining the data sets.

These combined transverse mass lineshapes were then used to fit the transverse mass distribution of the $W / W^{\prime} \rightarrow \mu \nu$ event candidates in the combined Run $1 \mathrm{~A}$ and Run 1B data set. The search results are shown in Table 7.2. The fit prefers non-zero $\beta$ values for low $M_{W^{\prime}}$, due to the small excess of events with $M_{T} \sim 200 \mathrm{GeV} / c^{2}$ above the background prediction, as shown in Figure 7.3. However, the excess is statistically insignificant.

In order to extract limits on the rate of $W^{\prime} \rightarrow \mu \nu$ production, it is convenient to deal with a likelihood distribution that is a function of $\beta$ only. A likelihood distribution as a function of $\beta$ only was obtained by fixing $\beta$ at a succession of points and minimising the negative log-likelihood at each point with respect to $\alpha_{0}$. The normalised likelihood distributions obtained this way for $250 \mathrm{GeV} / c^{2}$ and $700 \mathrm{GeV} / c^{2}$ $W^{\prime}$ bosons are shown in Figure 7.4. The peak away from $\beta=0$ for a $250 \mathrm{GeV} / c^{2} W^{\prime}$ boson indicates that the fit prefers a small non-zero $W^{\prime}$ boson fraction, as shown in Table 7.2 .

\footnotetext{
${ }^{4}$ The transverse mass lineshape of QCD background was normalised to the numbers of QCD background events obtained for each run as described in Section 6.4.
} 


\begin{tabular}{|c|c|}
\hline$M_{W^{\prime}}\left(\mathrm{GeV} / c^{2}\right)$ & Fraction of $W^{\prime} \rightarrow \mu \nu(\beta)$ \\
\hline \hline 200 & $0.009_{-0.004}^{+0.004}$ \\
250 & $0.011_{-0.006}^{+0.007}$ \\
300 & $0.006_{-0.006}^{+0.011}$ \\
350 & $0.000_{-0.000}^{+0.014}$ \\
400 & $0.000_{-0.000}^{+0.018}$ \\
450 & $0.000_{-0.000}^{+0.026}$ \\
500 & $0.000_{-0.000}^{+0.039}$ \\
550 & $0.000_{-0.000}^{+0.058}$ \\
600 & $0.000_{-0.000}^{+0.096}$ \\
650 & $0.000_{-0.000}^{+0.159}$ \\
700 & $0.000_{-0.000}^{+0.273}$ \\
750 & $0.000_{-0.000}^{+0.495}$ \\
800 & $0.000_{-0.000}^{+0.899}$ \\
\hline
\end{tabular}

Table 7.2: The results of the fit to the $M_{T}$ distribution of the $W / W^{\prime} \rightarrow \mu \nu$ event candidates in the Run $1 \mathrm{~A}+1 \mathrm{~B}$ data sample. The fit parameter $\beta$ is the fraction of $W^{\prime} \rightarrow \mu \nu$ production observed in data with respect to the expected number of observed $W^{\prime} \rightarrow \mu \nu$ events. The fit was performed in the physical region of $\beta$, i.e. $\beta \geq 0$. The uncertainties shown are statistical only. The systematic uncertainties are shown in Figure 7.7 and range from $\sim 0.04\left(M_{W^{\prime}}=200 \mathrm{GeV} / c^{2}\right)$ to $\sim 0.18\left(M_{W^{\prime}}=\right.$ $\left.800 \mathrm{GeV} / c^{2}\right)$. 

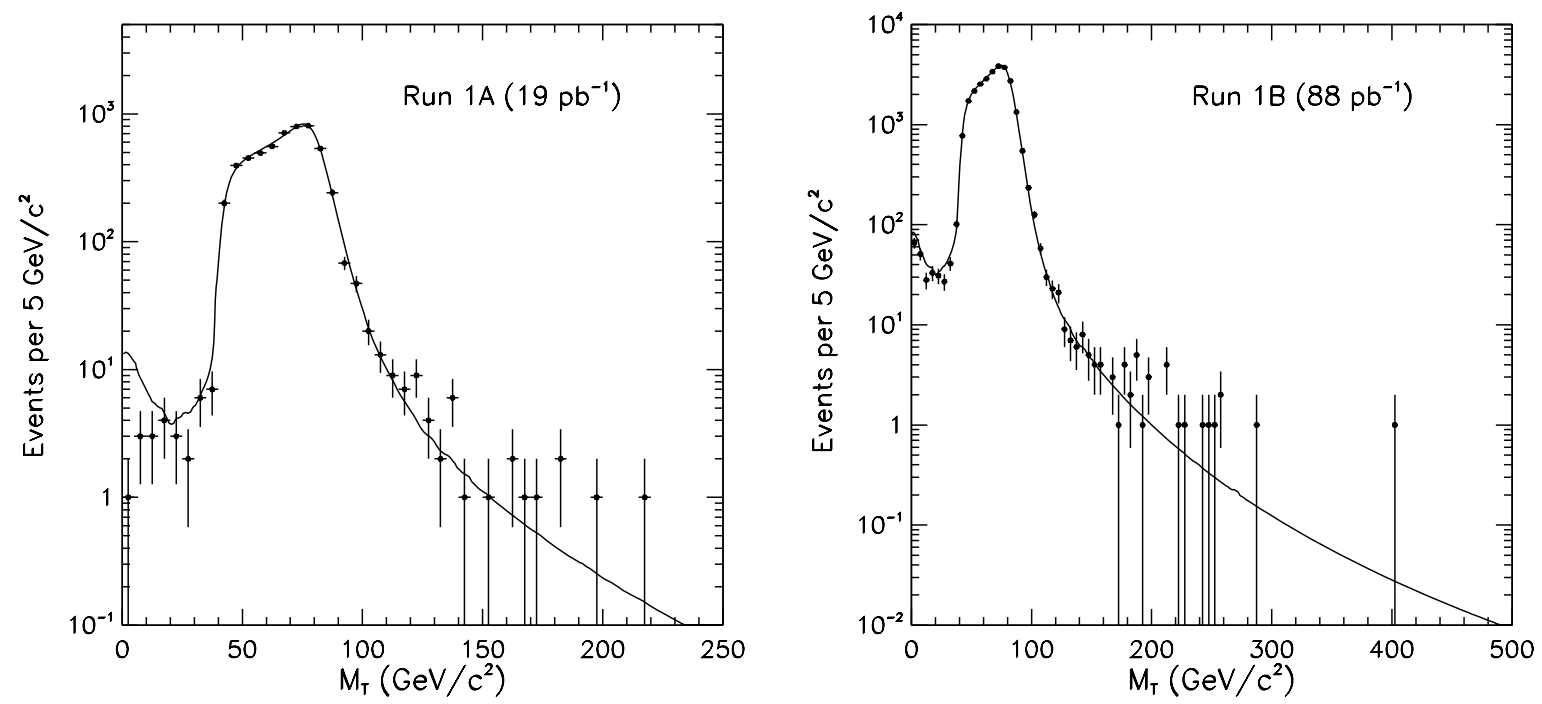

Figure 7.2: The transverse mass distribution of the $W / W^{\prime} \rightarrow \mu \nu$ event candidates (dots) overlaid with the standard model background prediction fit (solid line) in Run $1 \mathrm{~A}$ (left) and Run 1B (right). The disagreement between the prediction and data for $M_{T}<20 \mathrm{GeV} / c^{2}$ in the Run $1 \mathrm{~A}$ distribution arises from the use of the QCD background shape for Run 1B. However, this does not affect the overall fit because each fit was performed in the region of $40<M_{T}<2000 \mathrm{GeV} / c^{2}$.

\subsubsection{Fitting Bias}

It is important to verify that the fitting method can indeed identify a $W^{\prime} \rightarrow \mu \nu$ contribution, if it exists. In order to check the soundness of the method, a set of Monte Carlo "pseudo-experiments" was performed. A transverse mass distribution template of the background was produced by fitting the data with the nominal set of transverse mass lineshapes. ${ }^{5}$ The template for the expected signal was produced by setting $\alpha_{0}$ to be the value obtained from the background fit and $\beta=1$. A set of at least 1000 pseudo-experiments were performed for a given $M_{W^{\prime}}$ value, where in each experiment an observed transverse mass distribution was created using the template. The number of background events in each sample was fixed to the number of events obtained from the fit. The signal events were generated according to a Poisson distribution with the mean equal to the expected number of signal events. A

\footnotetext{
${ }^{5}$ Henceforth, the "nominal" set of transverse mass lineshapes refers to the set used to search for $W^{\prime} \rightarrow \mu \nu$ production as opposed to the sets of the transverse mass distribution lineshapes used to calculate the systematic uncertainties in Section 7.3.
} 


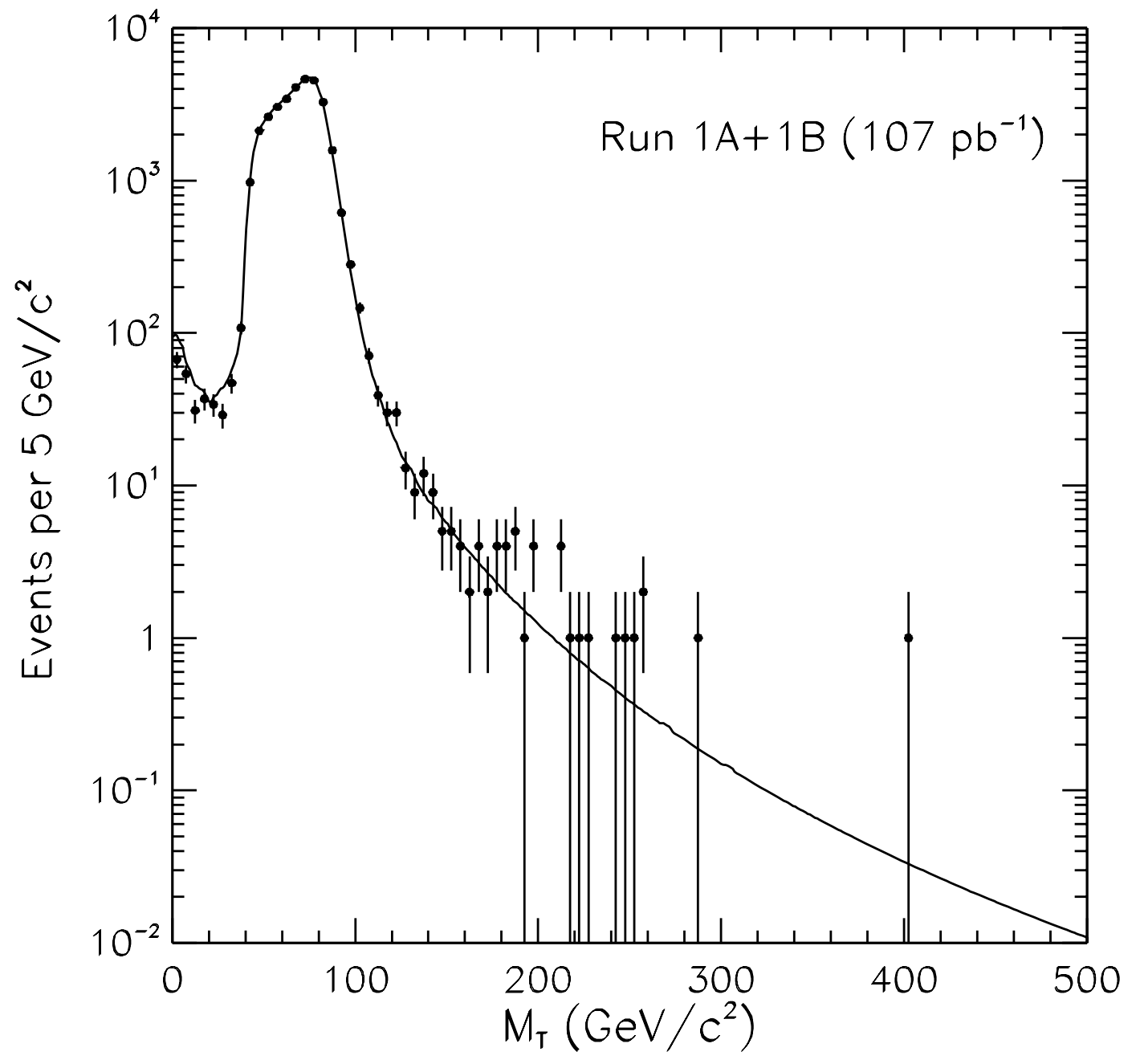

Figure 7.3: The transverse mass distribution of the $W / W^{\prime} \rightarrow \mu \nu$ event candidates in Run 1A+1B (dots) overlaid with the standard model background prediction fit (solid line). The fit was performed in the region of $40<M_{T}<2000 \mathrm{GeV} / c^{2}$. 

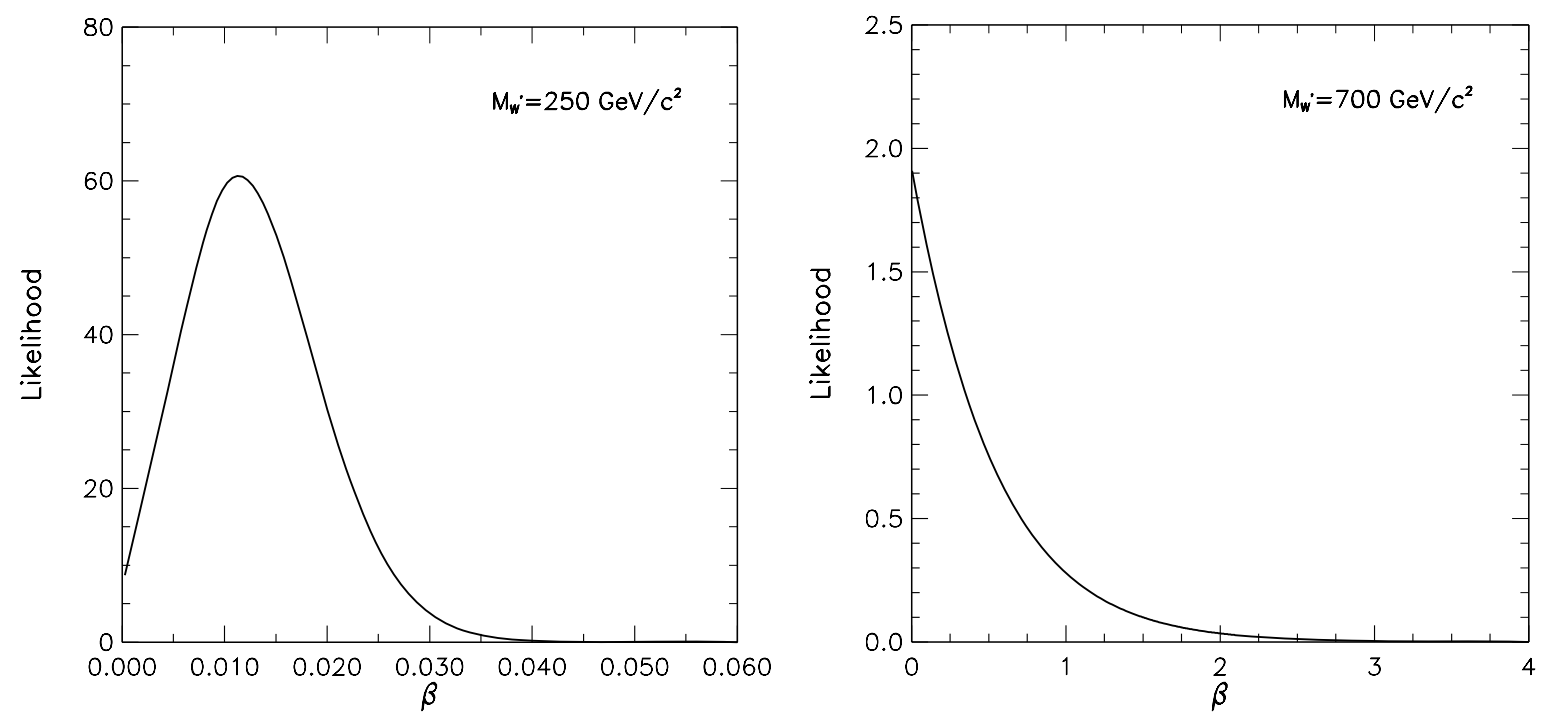

Figure 7.4: The likelihood distributions for $M_{W^{\prime}}=250 \mathrm{GeV} / c^{2}$ (left) and $M_{W^{\prime}}=$ $700 \mathrm{GeV} / \mathrm{c}^{2}$ (right) for Run $1 \mathrm{~A}+1 \mathrm{~B}$. The areas under the curves are normalised to unity.

fit was performed on each pseudo-experiment sample produced from the templates in the same manner as the data.

A summary of the results of the fits to the Monte Carlo transverse mass samples are shown in Table 7.3. The results show that the fitting method has a small bias in $\beta$ when the relative event rate of $W^{\prime} \rightarrow \mu \nu$ production is small compared to that of $W \rightarrow \mu \nu$ production, resulting in fitted values of $\beta$ that are slightly less than 1.0. This is due to the fact that the transverse mass distribution of $W^{\prime} \rightarrow \mu \nu$ events extends to the low transverse mass region that is heavily populated by the background events. The addition of small number of events from the $W^{\prime} \rightarrow \mu \nu$ contribution in the low transverse mass region results in fits that assign most of these to the much larger background contribution. This can be see in Figure 7.5, where the fit results for Monte Carlo samples created with a $700 \mathrm{GeV} / c^{2} W^{\prime}$ boson are shown. Except for the null signal event case, the mean number of signal events found by the fit, $\bar{n}_{f i t}$, is less than the number of actual signal events in the pseudo-experiment, $n_{\text {sig. }}$. The pile-up at $n_{f i t}=0$ for the cases where there are $n_{\text {sig }} \leq 2$ events in the sample is due to the fact that $\beta$ is constrained to the physical region, $\beta \geq 0$. Therefore, when the fit prefers a negative value of $\beta, \beta$ is forced to be 0 . This bias from the fitting method 


\begin{tabular}{|c|c|}
\hline$M_{W^{\prime}}\left(\mathrm{GeV} / c^{2}\right)$ & $\beta_{0}$ \\
\hline \hline 200 & 1.00 \\
250 & 1.00 \\
300 & 1.00 \\
350 & 1.00 \\
400 & 1.00 \\
450 & 1.00 \\
500 & $9.99 \times 10^{-1}$ \\
550 & $9.96 \times 10^{-1}$ \\
600 & $9.88 \times 10^{-1}$ \\
650 & $9.72 \times 10^{-1}$ \\
700 & $9.52 \times 10^{-1}$ \\
750 & $9.44 \times 10^{-1}$ \\
800 & $9.62 \times 10^{-1}$ \\
\hline
\end{tabular}

Table 7.3: The biases in $\beta, \beta_{0}$, from the fitting method. The $\beta_{0}$ is the mean value of $\beta$ determined from the Monte Carlo pseudo-experiments (see text).

was compensated for when calculating the limits on $\beta$ (see Section 7.5).

\subsection{Systematic Uncertainties}

Systematic uncertainties in the fit results come from various sources of the uncertainties in the theoretical calculations and the measured quantities incorporated into the determination of the efficiency times acceptance of the events. The dominant systematic uncertainty comes from the choice of parton distribution function sets (see Section 7.3.2), which is independent of the data taking period (i.e. Run $1 \mathrm{~A}$ and Run 1B). The uncertainties from the boson $p_{T}$ scale and the relative production rate of background processes are independent of the data set. Other systematic uncertainties are expected to be similar in Run $1 \mathrm{~A}$ and Run 1B. Therefore, effects from the differences in the systematic uncertainties between the Run $1 \mathrm{~A}$ and the Run $1 \mathrm{~B}$ analyses are expected to be small, especially when limits on $\beta$ are large compared to the systematic uncertainties. Also, the Run $1 \mathrm{~A}$ data constitutes only $18 \%$ of the combined Run $1 \mathrm{~A}$ and Run 1B data in terms of the number of $W / W^{\prime} \rightarrow \mu \nu$ event candidates. Thus the systematic uncertainties calculated for the Run 1B data were used for Run 

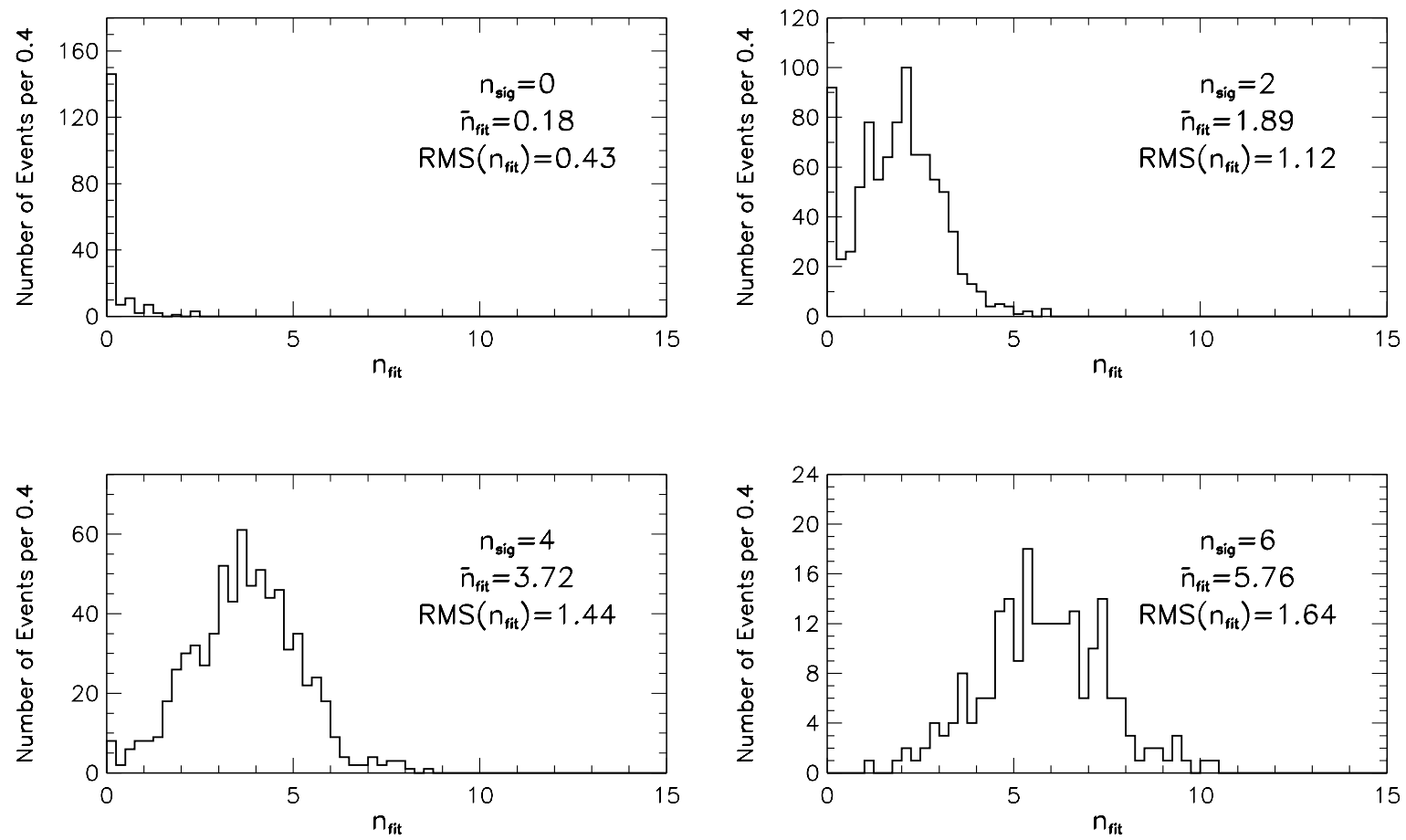

Figure 7.5: The distribution of the number of signal events found by the fit $\left(n_{f i t}\right)$ in background+signal pseudo-experiments with $700 \mathrm{GeV} W^{\prime}$ boson for different number of actual signal events $\left(n_{\text {sig }}\right)$ inserted into the background sample. 
$1 \mathrm{~A}$ and Run 1B combined data instead of calculating them independently for the Run $1 \mathrm{~A}$ sample. This is a conservative assumption but simplifies the combined analysis.

\subsubsection{Systematic Uncertainty Calculation}

To account for the effects of systematic uncertainties on the limits set on $\beta$, a set of Monte Carlo pseudo-experiments similar to those described in Section 7.2.4 was performed. Normalised transverse mass lineshapes were produced with a given source of uncertainty varied, such as different parton distribution function sets. The transverse mass distribution templates for the background and the signal were produced from a fit to the data. Then at least 1000 pseudo-experiments were generated for a given $M_{W^{\prime}}$ according to these templates. The transverse mass distribution from each pseudo-experiment was fitted with the nominal set of transverse mass lineshapes to determine the mean value for $\beta$. This resulted in shifts in $\beta$ that reflected how the systematic uncertainty affected $\beta$. The difference between the mean $\beta$ of the set being tested and that of the nominal set, $\Delta \beta$, was calculated as

$$
\Delta \beta=\left(\left|\beta_{+}-\beta_{0}\right|+\left|\beta_{-}-\beta_{0}\right|\right) / 2
$$

where $\beta_{0}$ is the fit value from the pseudo-experiment that was generated from the transverse mass distribution templates produced by fitting the data with the nominal set of transverse mass lineshapes, and $\beta_{ \pm}$is the fit value from the pseudo-experiments with $\pm 1 \sigma$ systematic uncertainty variations. ${ }^{6}$

The entire procedure was repeated for each systematic effect. Finally, $\Delta \beta$ from each systematic effect was summed in quadrature to obtain the total systematic uncertainty in $\beta, \Delta \bar{\beta}$. A Gaussian distribution was used to smear the observed likelihood distribution, $L(\beta)$, with $\Delta \bar{\beta}$ as its RMS deviation,

$$
L^{\prime}(\beta)=\int_{0}^{\infty} L\left(\beta^{\prime}\right) \exp \left(-\frac{\left(\beta-\beta^{\prime}\right)^{2}}{2(\Delta \bar{\beta})^{2}}\right) d \beta^{\prime}
$$

to obtained the smeared likelihood distribution, $L^{\prime}(\beta)$. The smearing was performed

\footnotetext{
${ }^{6}$ Except for the choice of parton distribution function set, as will be seen in Section 7.3.2.
} 
only in the physical region, i.e. $\beta \geq 0$.

\subsubsection{Systematic Uncertainty Effects}

The systematic effects that were considered are listed here.

\section{Choice of Parton Distribution Functions}

The systematic uncertainty from the choice of parton distribution function set comes primarily from the difference in the ratio of the cross sections of $W^{\prime}$ and $W$ bosons and secondarily from the differences in shapes of the predicted transverse mass distributions. In Figure 7.6, the ratios of $\sigma \cdot \mathcal{B}\left(W^{\prime} \rightarrow \mu \nu\right)$ to $\sigma \cdot \mathcal{B}(W \rightarrow \mu \nu)$ for various parton distribution function sets with $M_{W^{\prime}}=650 \mathrm{GeV} / c^{2}$ are shown. To estimate the systematic uncertainty due to the choice of parton distribution function set, the MRS-R2 [56] and MRS-H ( $\overline{\mathrm{MS}}$ ) [61] next-to-leading order parton distribution function sets were used to generate $W \rightarrow \mu \nu, W \rightarrow \tau \nu, Z / \gamma \rightarrow \mu \mu, Z / \gamma \rightarrow \tau \tau$, and $W^{\prime} \rightarrow \mu \nu$ events. This resulted in uncertainties ranging from $\sim 5 \%$ for $M_{W^{\prime}}=200 \mathrm{GeV} / c^{2}$ to $\sim 12 \%$ for $M_{W^{\prime}}=800 \mathrm{GeV} / c^{2}$.

\section{Muon Momentum Resolution}

The muon $p_{T}$ resolution (see Eq. (5.6)) was varied by $\pm 1 \sigma$ and the Monte Carlo events were reanalysed. This resulted in uncertainties ranging from $\sim 0.4 \%$ to $\sim 8 \%$.

\section{$W / W^{\prime} \operatorname{Boson} p_{T}$}

The $W / W^{\prime}$ boson $p_{T}$ distribution was scaled by $\pm 10 \%$ to model the uncertainty in the production mechanism for $W$ and $W^{\prime}$ bosons. This resulted in uncertainties ranging from $\sim 0.8 \%$ to $\sim 9 \%$.

\section{Relative Production Rate}

The relative production rates of various background components were varied individually by $\pm 1 \sigma$. Table 7.4 shows the one standard deviation variations of the relative 


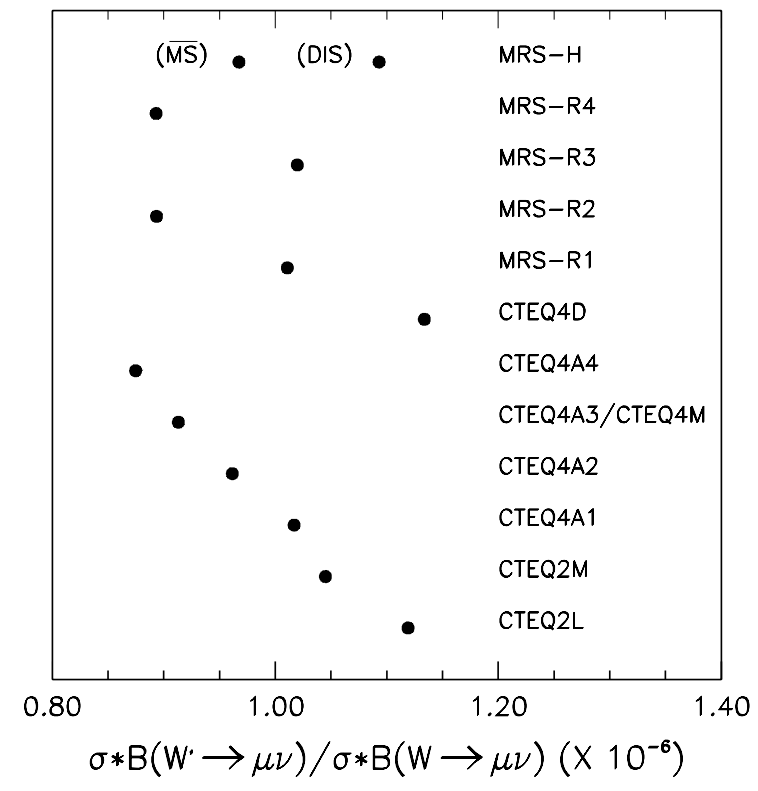

Figure 7.6: The ratios of $\sigma \cdot \mathcal{B}\left(W^{\prime} \rightarrow \mu \nu\right)$ to $\sigma \cdot \mathcal{B}(W \rightarrow \mu \nu)$ for various next-to-leading order parton distribution function sets (except for CTEQ2L, which is leading-order) for a $650 \mathrm{GeV} / c^{2} W^{\prime}$ boson $[16,56,60,61]$. The cross sections were calculated within $\pm 6 \Gamma\left(W / W^{\prime}\right)$ of the mass values. For the systematic uncertainty calculation, MRS$\mathrm{H}(\overline{\mathrm{MS}})$ and MRS-R2 parton distribution function sets were used. 


\begin{tabular}{|c|c|}
\hline Background & Fraction Varied \\
\hline \hline$Z / \gamma \rightarrow \mu \mu$ & \pm 0.04 \\
$Z / \gamma \rightarrow \tau \tau$ & \pm 0.04 \\
$t \bar{t}$ production & $+0.24 /-0.20$ \\
QCD & \pm 0.49 \\
\hline
\end{tabular}

Table 7.4: Summary of the uncertainties in the relative rates of the backgrounds. Fractions of $Z / \gamma \rightarrow \mu \mu$ and $Z / \gamma \rightarrow \tau \tau$ were varied together.

production rate of the background sources. The resulting changes were added in quadrature. This resulted in uncertainties ranging from $\sim 0.3 \%$ to $\sim 3 \%$.

\section{Muon Selection Efficiency}

The muon selection efficiencies for $\mathrm{CMU} / \mathrm{P}$ muons and CMX muons were varied by $\pm 1 \sigma$. When the $\mathrm{CMU} / \mathrm{P}$ muon selection efficiency was varied, the CMX muon efficiency was fixed at the nominal value and vice versa. This resulted in uncertainties ranging from $\sim 0.8 \%$ to $\sim 2 \%$.

\section{Trigger Efficiencies}

The combined efficiencies of the Level 1 and Level 2 triggers were varied by $\pm 1 \sigma$ in the same manner as the muon selection efficiency. This resulted in uncertainties ranging from $\sim 0.3 \%$ to $\sim 3 \%$.

\section{Underlying Event Model}

The widths of the $u_{1}$ and $u_{2}$ distributions were varied by $\pm 1 \sigma$. This resulted in uncertainties ranging from $\sim 0.3 \%$ to $\sim 5 \%$.

\section{Summary of Systematic Uncertainties}

The systematic uncertainties $(\Delta \beta)$ are shown as functions of $M_{W}$ in Figure 7.7. The total systematic uncertainty varies from $\sim 4 \%$ at $M_{W^{\prime}}=200 \mathrm{GeV} / c^{2}$ to $\sim 18 \%$ at $M_{W^{\prime}}=800 \mathrm{GeV} / c^{2}$. In most of the $M_{W^{\prime}}$ region, the systematic uncertainty due to the 
choice of parton distribution function set dominates the total systematic uncertainties. The uncertainty stemming from the scaling of the Run 1A transverse mass lineshape with respect to that of Run $1 \mathrm{~B}$ is small compared to other systematic uncertainties and was ignored.

The likelihood distributions for $\beta$ for a $200 \mathrm{GeV} / c^{2} W^{\prime}$ boson and a $650 \mathrm{GeV} / c^{2} W^{\prime}$ boson before and after the systematic uncertainty smearing are shown in Figure 7.8 . For a $200 \mathrm{GeV} / \mathrm{c}^{2} W^{\prime}$ boson, the smearing is large compared to the statistical precision by which $\beta$ is determined. For a $650 \mathrm{GeV} / c^{2} W^{\prime}$ boson, the smearing effects are visible only near $\beta=0$. A peak away from $\beta=0$ appears after the smearing because of the constraint, $\beta \geq 0$, in the systematic uncertainty smearing procedure: A point in physical region $(\beta \geq 0)$ can move into the unphysical region $(\beta<0)$ but not the other way around.

\subsection{Background Contribution Estimate}

The contributions from individual background components were obtained from the fit. Table 7.5 shows the expected numbers of observed contributions in Run $1 \mathrm{~A}+1 \mathrm{~B}$ in the transverse mass region dominated by backgrounds $\left(40<M_{T}<200 \mathrm{GeV} / c^{2}\right)$ and the high transverse mass region $\left(M_{T}>200 \mathrm{GeV} / c^{2}\right)$ where the expected number of background events is small. The uncertainties were obtained with the same procedure described in Appendix A. The uncertainties are correlated. The expected total number of observed background at $M_{T}>200 \mathrm{GeV} / c^{2}$ is $11.8 \pm 0.9$ and is in agreement with the data, which has 14 events in the same transverse mass region (see Table 3.1 ).

\subsection{Limits}

The limit on $\beta$ at $95 \%$ confidence level (CL), $\beta_{95}$, is calculated using the formula

$$
0.95=\frac{1}{\int_{0}^{\infty} L(\beta) d \beta} \int_{0}^{\beta_{95}^{\prime}} L(\beta) d \beta
$$




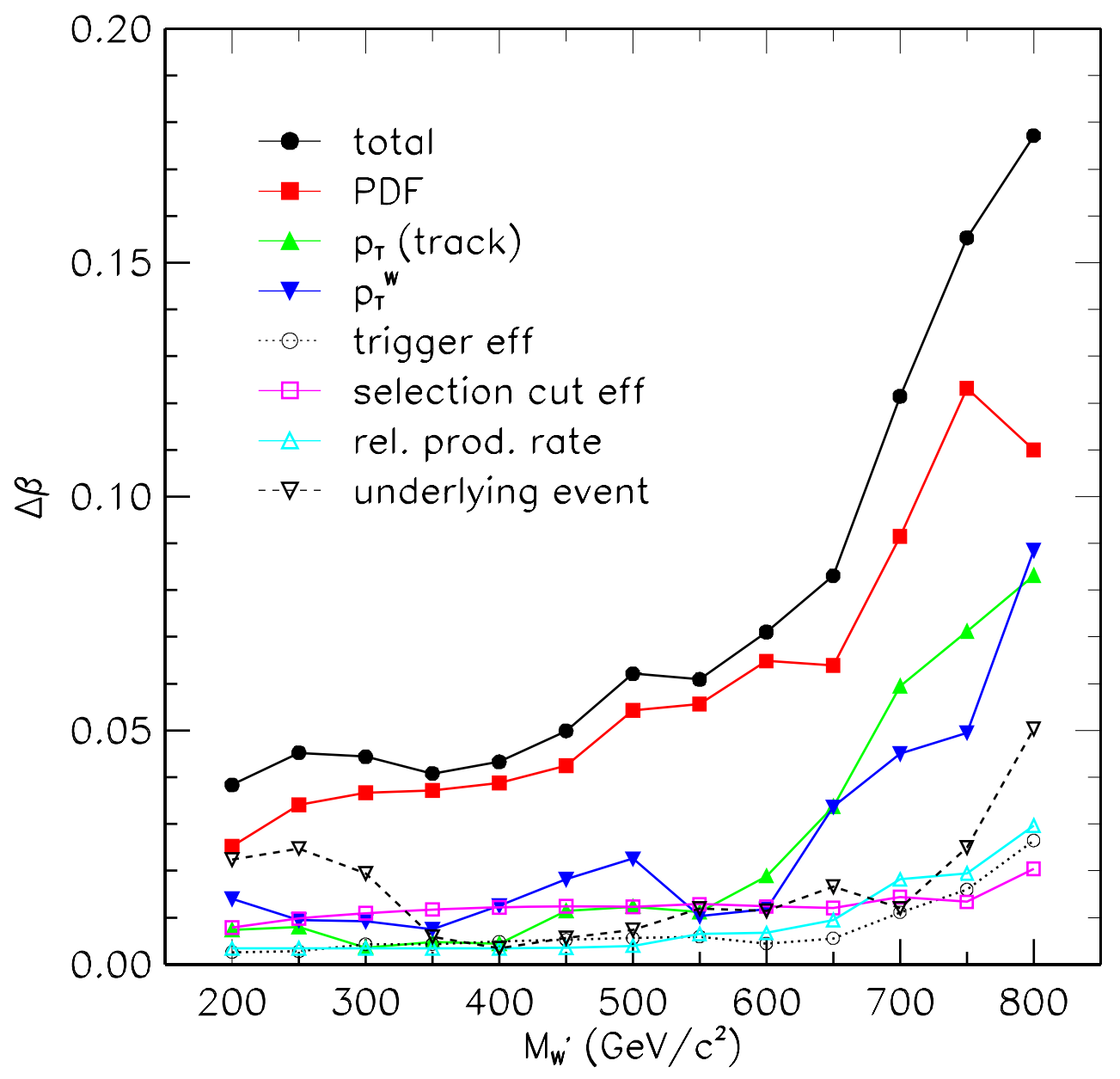

Figure 7.7: The systematic uncertainties $(\Delta \beta)$ as a function of $M_{W^{\prime}}$. The total systematic uncertainty is a sum in quadrature of the individual systematic uncertainties.

\begin{tabular}{|c|cc|}
\hline Background & \multicolumn{2}{|c|}{ Number of Events } \\
& $\left(40<M_{T}<200 \mathrm{GeV} / c^{2}\right)$ & $\left(M_{T}>200 \mathrm{GeV} / c^{2}\right)$ \\
\hline \hline$W \rightarrow \mu \nu$ & $27925 \pm 209$ & $8.99 \pm 0.81$ \\
$Z / \gamma \rightarrow \mu \mu$ & $2824 \pm 196$ & $2.02 \pm 0.35$ \\
$W \rightarrow \tau \nu$ & $687 \pm 27$ & $0.04 \pm 0.01$ \\
$Z / \gamma \rightarrow \tau \tau$ & $47 \pm 3$ & $0.02 \pm 0.02$ \\
$t \bar{t}$ Production & $14_{-3}^{+4}$ & $0.29_{-0.06}^{+0.07}$ \\
QCD Background & $74 \pm 37$ & $0.42_{-0.42}^{+0.43}$ \\
\hline
\end{tabular}

Table 7.5: The contributions from individual background components. 

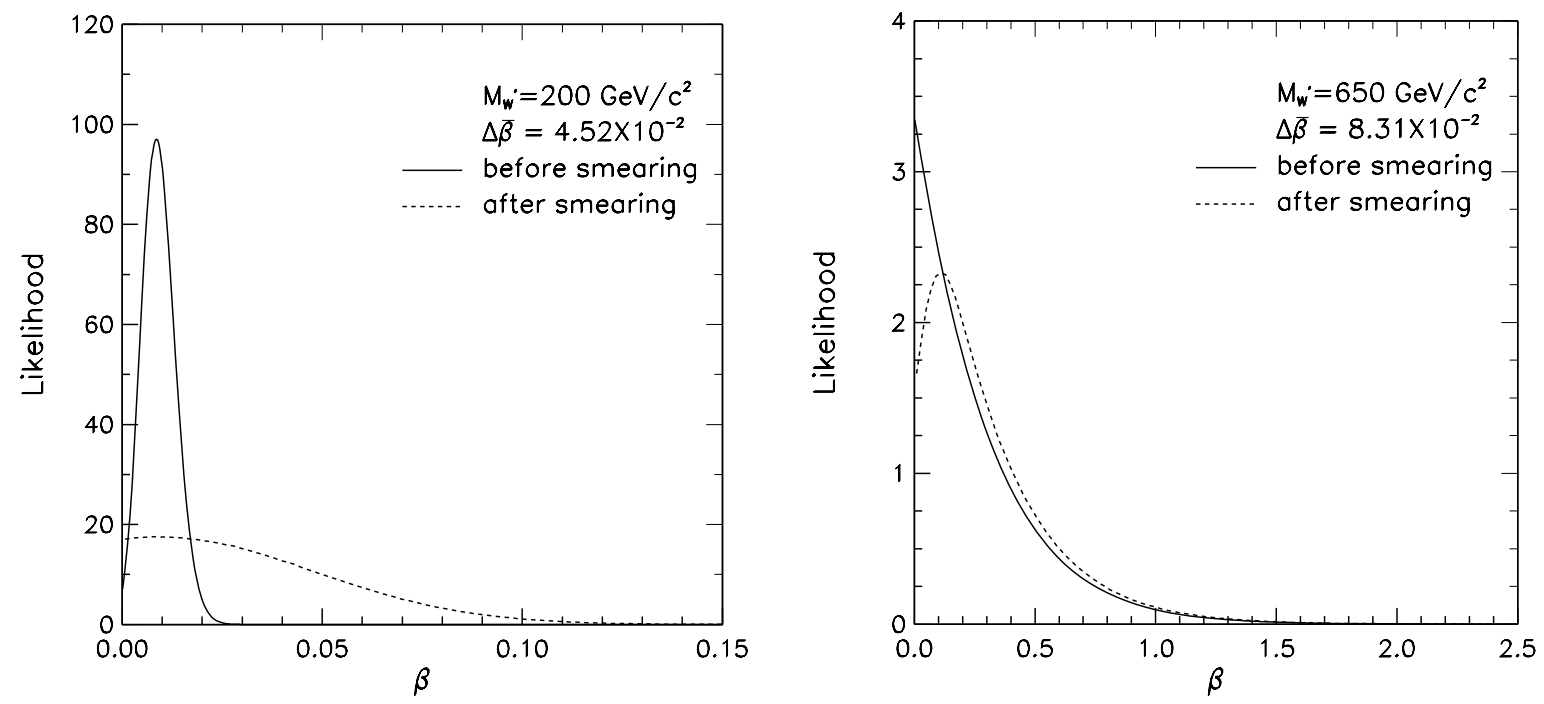

Figure 7.8: The likelihood distributions of $M_{W^{\prime}}=200 \mathrm{GeV} / c^{2}$ (left) and $M_{W^{\prime}}=$ $650 \mathrm{GeV} / \mathrm{c}^{2}$ (right) before and after the systematic uncertainty smearing. The areas under the curves are normalised to unity.

The bias from the fitting method described in Section 7.2.4 is corrected by defining $\beta_{95}$ as

$$
\beta_{95} \equiv \beta_{95}^{\prime} / \beta_{0}
$$

The values of $\beta_{95}$ with and without the systematic uncertainty smearing are shown in Table 7.6.

Since $\beta_{95}$ is the limit on the fraction of the number of $W^{\prime} \rightarrow \mu \nu$ events expected for a $W^{\prime}$ with the standard model strength couplings and branching fraction, the limits on the cross section times branching fraction for $W^{\prime} \rightarrow \mu \nu$ production at $95 \%$ confidence level (CL) are calculated using the relationship

$$
\left(\sigma \cdot \mathcal{B}\left(W^{\prime} \rightarrow \mu \nu\right)\right)_{95 \% C L}=\beta_{95} \cdot\left(\sigma \cdot \mathcal{B}\left(W^{\prime} \rightarrow \mu \nu\right)\right)_{S M},
$$

where $\left(\sigma \cdot \mathcal{B}\left(W^{\prime} \rightarrow \mu \nu\right)\right)_{S M}$ is the cross section times branching fraction of $W^{\prime} \rightarrow \mu \nu$ with the standard model strength couplings, branching fraction, and the CKM matrix. The limit on the cross section times branching fraction as a function of $M_{W}$ is shown in Figure 7.9 where the region above the curve is excluded at 95\% CL. Also the prediction for $W^{\prime} \rightarrow \mu \nu$ production with the standard model strength couplings and 


\begin{tabular}{|c|cc|cc|}
\hline$M_{W^{\prime}}$ & \multicolumn{2}{|c|}{$\beta_{95}$} & \multicolumn{2}{c|}{$\sigma \cdot \mathcal{B}\left(W^{\prime} \rightarrow \mu \nu\right)(\mathrm{pb})$} \\
$\left(\mathrm{GeV} / \mathrm{c}^{2}\right)$ & (without smearing) & (with smearing) & SM Strength & $95 \% \mathrm{CL}$ \\
\hline \hline 200 & $1.64 \times 10^{-2}$ & $8.22 \times 10^{-2}$ & $1.40 \times 10^{2}$ & $<1.13 \times 10^{1}$ \\
250 & $2.49 \times 10^{-2}$ & $9.90 \times 10^{-2}$ & $5.71 \times 10^{1}$ & $<5.54$ \\
300 & $3.00 \times 10^{-2}$ & $9.82 \times 10^{-2}$ & $2.60 \times 10^{1}$ & $<2.49$ \\
350 & $3.86 \times 10^{-2}$ & $9.45 \times 10^{-2}$ & $1.28 \times 10^{1}$ & $<1.17$ \\
400 & $5.58 \times 10^{-2}$ & $1.08 \times 10^{-1}$ & 6.57 & $<6.91 \times 10^{-1}$ \\
450 & $8.92 \times 10^{-2}$ & $1.40 \times 10^{-1}$ & 3.50 & $<4.81 \times 10^{-1}$ \\
500 & $1.50 \times 10^{-1}$ & $2.03 \times 10^{-1}$ & 1.90 & $<3.83 \times 10^{-1}$ \\
550 & $2.58 \times 10^{-1}$ & $2.97 \times 10^{-1}$ & 1.05 & $<3.10 \times 10^{-1}$ \\
600 & $4.61 \times 10^{-1}$ & $5.02 \times 10^{-1}$ & $5.86 \times 10^{-1}$ & $<2.93 \times 10^{-1}$ \\
650 & $8.39 \times 10^{-1}$ & $8.83 \times 10^{-1}$ & $3.31 \times 10^{-1}$ & $<2.92 \times 10^{-1}$ \\
700 & 1.54 & 1.60 & $1.90 \times 10^{-1}$ & $<3.04 \times 10^{-1}$ \\
750 & 2.86 & 2.94 & $1.10 \times 10^{-1}$ & $<3.24 \times 10^{-1}$ \\
800 & 5.17 & 5.27 & $6.57 \times 10^{-2}$ & $<3.46 \times 10^{-1}$ \\
\hline
\end{tabular}

Table 7.6: The $95 \%$ confidence level limits of $\beta, \beta_{95}$, with and without the systematic uncertainty smearing, and the cross sections times branching fraction of $W^{\prime} \rightarrow \mu \nu$ with the standard model (SM) strength couplings and their 95\% CL limits.

branching fraction is shown. Consequently, the limit on the $W^{\prime}-q-q$ coupling (see Section 1.3) times branching fraction of $W^{\prime} \rightarrow \mu \nu$ can be written as

$$
\beta_{95}=\frac{\left(\lambda^{2} \cdot \mathcal{B}\left(W^{\prime} \rightarrow \mu \nu\right)\right)_{95 \% C L}}{\left(\lambda^{2} \cdot \mathcal{B}\left(W^{\prime} \rightarrow \mu \nu\right)\right)_{S M}}
$$

where $\left(\lambda^{2} \cdot \mathcal{B}\left(W^{\prime} \rightarrow \mu \nu\right)\right)_{S M}$ is the standard model strength couplings times branching fraction. Figure 7.10 shows the region of $\left(\lambda^{2} \cdot \mathcal{B}\right) /\left(\lambda^{2} \cdot \mathcal{B}\right)_{S M}$ excluded at $95 \% \mathrm{CL}$ as a function of $M_{W^{\prime}}$. The limits are summarised in Table 7.6. For a $W^{\prime}$ boson with the standard model strength couplings and branching fraction, these limits imply that

$$
M_{W^{\prime}}>660 \mathrm{GeV} / c^{2}(\text { at } 95 \% \mathrm{CL})
$$

A cross-check was performed on the Run $1 \mathrm{~B}$ results by performing a "counting experiment" to set the mass limit of a $W^{\prime}$ boson. This is discussed in Appendix A. The limits from Run $1 \mathrm{~A}$ and Run 1B separately are shown in Appendix B. 


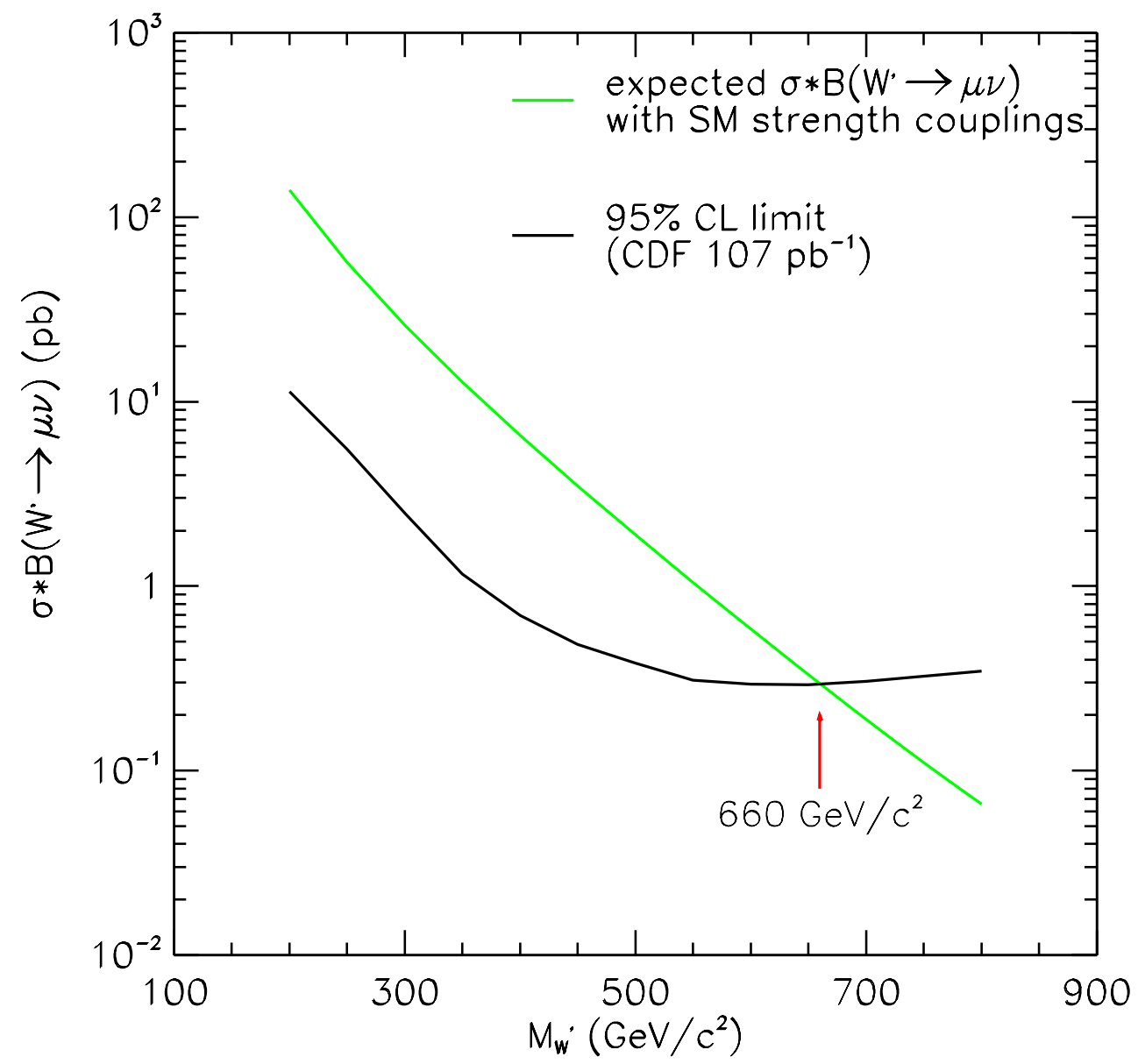

Figure 7.9: The $95 \% \mathrm{CL} \sigma \cdot \mathcal{B}\left(W^{\prime} \rightarrow \mu \nu\right)$ limit. The region above the curve is excluded. Also shown is the $\sigma \cdot \mathcal{B}\left(W^{\prime} \rightarrow \mu \nu\right)$ curve for the standard model strength couplings and the branching fraction. The intercept yields $M_{W^{\prime}}>660 \mathrm{GeV} / c^{2}$ at $95 \% \mathrm{CL}$. 


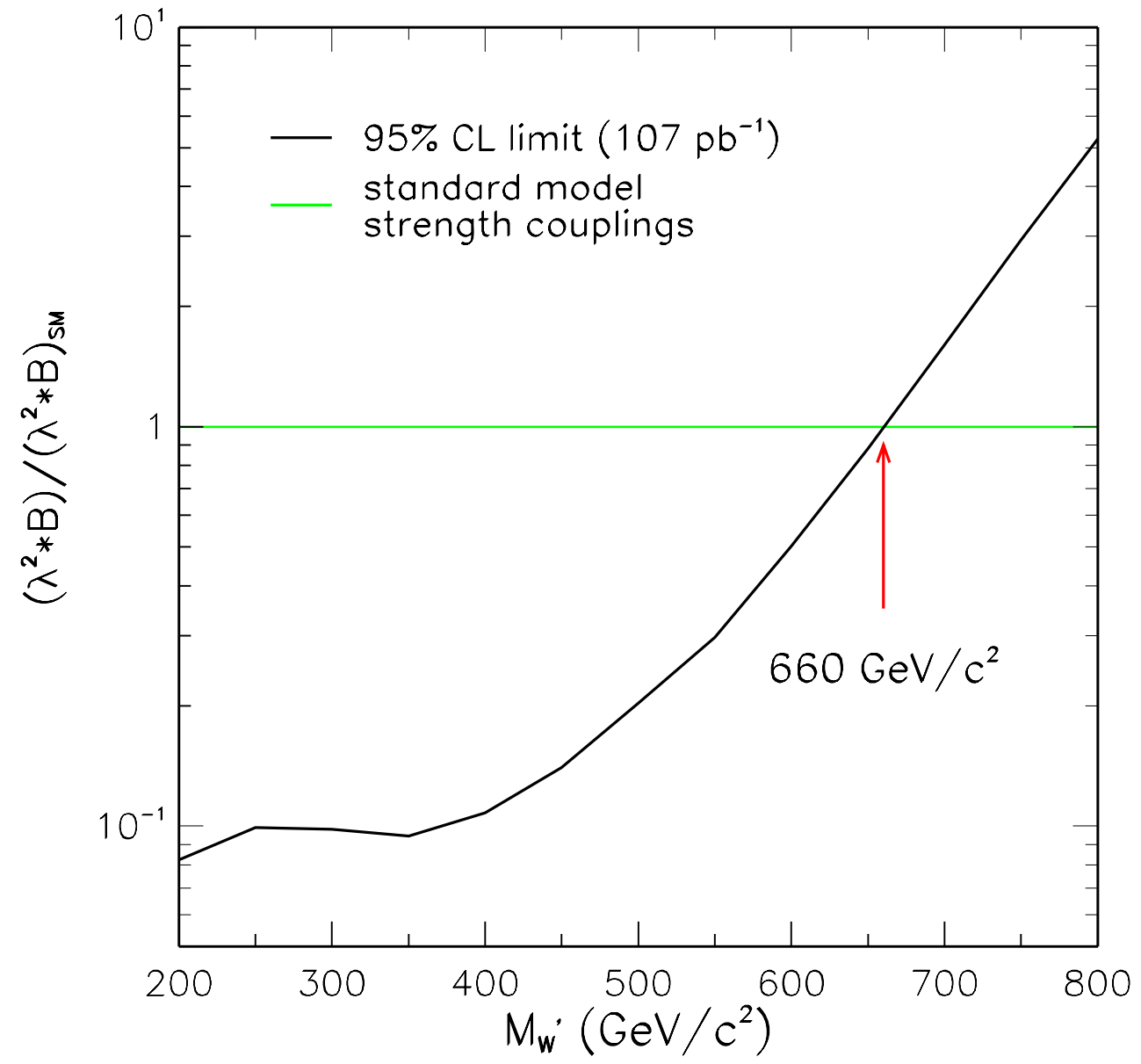

Figure 7.10: The 95\% CL limit on the ratio of coupling strength times branching fraction, $\lambda^{2} \cdot \mathcal{B}$. The region above the $\left(\lambda^{2} \cdot \mathcal{B}\right) /\left(\lambda^{2} \cdot \mathcal{B}\right)_{S M}=\beta_{95}$ curve is excluded at $95 \%$ CL. For the standard model strength couplings and branching fraction, $\left(\lambda^{2}\right.$. $\mathcal{B}) /\left(\lambda^{2} \cdot \mathcal{B}\right)_{S M}=1$. The arrow shows the resulting $95 \%$ CL mass limit for the $W^{\prime}$ boson, $M_{W^{\prime}}>660 \mathrm{GeV} / c^{2}$, assuming these couplings. 


\section{Chapter 8}

\section{Conclusions}

\subsection{Summary of Results}

The new charged vector boson $W^{\prime}$ was searched for in the $W^{\prime} \rightarrow \mu \nu$ decay channel using a data sample recorded by the Collider Detector at Fermilab corresponding to an integrated luminosity of $(107 \pm 7) \mathrm{pb}^{-1}$ of $p \bar{p}$ collisions at $\sqrt{s}=1.8 \mathrm{TeV}$ at the Tevatron Collider at Fermilab. No evidence of $W^{\prime} \rightarrow \mu \nu$ production was found and

limits were set on the coupling strength times branching fraction, $\lambda^{2} \cdot \mathcal{B}\left(W^{\prime} \rightarrow \mu \nu\right)$, and, conversely, the production cross section times branching fraction, $\sigma\left(p \bar{p} \rightarrow W^{\prime}\right)$. $\mathcal{B}\left(W^{\prime} \rightarrow \mu \nu\right)$. These limits are shown in Figures 7.9 and 7.10 , respectively. Assuming the standard model strength couplings and branching fraction, the lower mass limit of a $W^{\prime}$ boson is

$$
M_{W^{\prime}}>660 \mathrm{GeV} / c^{2}(\text { at } 95 \% \mathrm{CL})
$$

This is the most stringent direct limit set for a $W^{\prime}$ boson coupling to the $\mu \nu$ final state and is competitive with the indirect measurements. Also, we can conclude that this channel is not background limited yet, as the expected yield at high transverse mass comes from the irreducible background from the $W \rightarrow \mu \nu$ process.

This result can be converted into the limit on the event rate of low energy processes such as muon decay due to the $V+A$ interaction. The event rate of such a process is proportional to $\left(1 / M_{W^{\prime}}\right)^{4}$. Therefore the ratio of event rates due to $V+A$ interactions to that of $V-A$ interactions is $\left(M_{W} / M_{W^{\prime}}\right)^{4}$. The result in Eq. 8.1 implies a limit on 
the ratio

$$
\frac{n(V+A)}{n(V-A)}<2.2 \times 10^{-4}(\text { at } 95 \% \mathrm{CL}),
$$

where $n(V+A)$ and $n(V-A)$ are the event rates due to the $V+A$ and $V-A$ interactions, respectively.

\subsection{Future Prospects}

The Tevatron is being upgraded with a "Main Injector" and is expected to deliver an instantaneous luminosity of $2 \times 10^{32} \mathrm{~cm}^{-2} \mathrm{~s}^{-1}$ at a center-of-mass energy of $\sqrt{s}=2 \mathrm{TeV}$ starting in 2000. This "Run 2" is scheduled to deliver a total integrated luminosity of $2 \mathrm{fb}^{-1}$ to an upgraded CDF detector. At $\sqrt{s}=2 \mathrm{TeV}$, the production cross section of $900 \mathrm{GeV} / c^{2} W^{\prime}$ bosons is expected to be $\sim 1.8$ times that of the cross section at $\sqrt{s}=1.8 \mathrm{TeV}$. With $\sim 20$ times more integrated luminosity and the higher center-ofmass energy, the range of the $W^{\prime} \rightarrow \mu \nu$ search can be expanded to $\sim 900 \mathrm{GeV} / c^{2}$, as shown in Figure 8.1, which is an extrapolation of the past and the current searches in the muon decay channel. ${ }^{1}$

In Refs. [23] and [24], indirect limits on the mass of a $W^{\prime}\left(W_{R}\right)$ boson were inferred from a study of the positron asymmetry, $\xi P_{\mu} \delta / \rho$, in the decay of a polarised muon, $\mu^{+} \rightarrow e^{+} \nu_{e} \bar{\nu}_{\mu}$, where $\xi, \delta$, and $\rho$ are the Michel parameters, and $P_{\mu}$ is the muon polarisation. Conversely, one can exclude a region of $\xi P_{\mu} \delta / \rho$ from the $W^{\prime}$ boson mass limit presented in this thesis. Assuming no $W_{L}-W_{R}$ mixing, the limit on the positron asymmetry is found to be

$$
\xi P_{\mu} \delta / \rho=1-4\left(\frac{M_{W_{L}}}{M_{W_{R}}}\right)^{4}>0.9991(\text { at } 95 \% \mathrm{CL})
$$

This result is comparable to the sensitivity of the planned muon decay experiment at TRIUMF, E614 [62].

\footnotetext{
${ }^{1}$ The increase in the center-of-energy has almost the same effect as the increase in the integrated luminosity. Therefore, the expected range of the $W^{\prime} \rightarrow \mu \nu$ search at $\sqrt{s}=2 \mathrm{TeV}$ with $2 \mathrm{fb}^{-1}$ would be about the same as the expected range of the search at $\sqrt{s}=1.8 \mathrm{TeV}$ with $\sim 3.6 \mathrm{fb}^{-1}$ in Figure 8.1.
} 


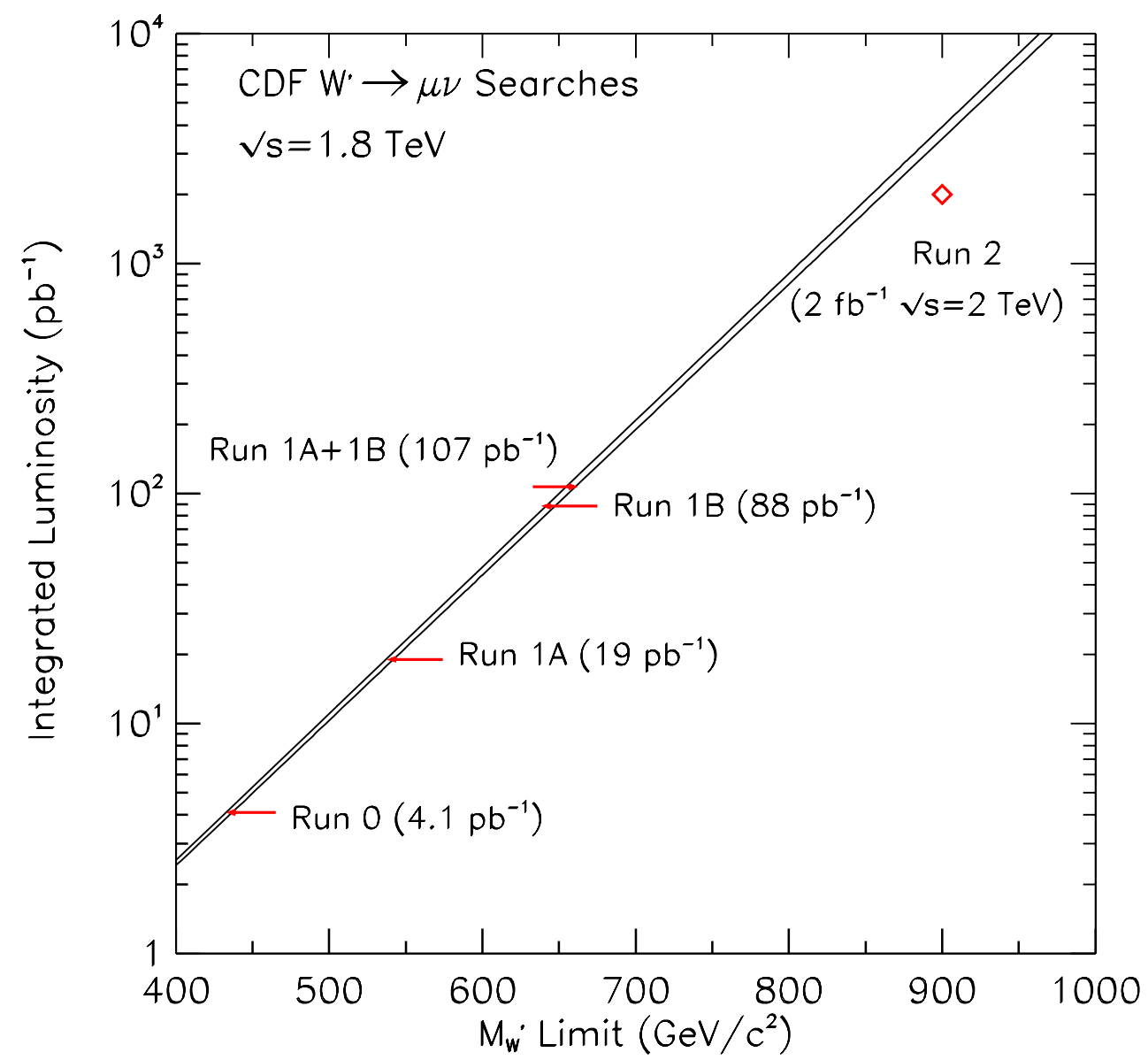

Figure 8.1: The integrated luminosity versus prospective $W^{\prime}$ mass limit from a $W^{\prime} \rightarrow$ $\mu \nu$ search at CDF. The Run 2 limit was extrapolated from the curve assuming $\sqrt{s}=$ $2 \mathrm{TeV}$. See Appendix B for the limits from the individual data sets. The "Run 0" limit is from Ref. [20]. 


\section{Appendix A}

\section{Cross-Check: Counting Experiment}

The lower mass limit for a $W^{\prime}$ boson for the Run 1B data set was extracted from counting the number of events in the high transverse mass region where the expected number of background events is small. The limits obtained from the counting experiment should be comparable to the limits presented in Section 7.5, and represent a cross-check of the technique used in the analysis. This technique does suffer from larger systematic uncertainties and the arbitrary signal region selection.

A probability of observing less than or equal to $n_{o}$ events with the expected number of background events, $\mu_{B}$ with its uncertainty $\Delta \mu_{B}$, and the expected number of signal events, $\mu_{S}$ with its uncertainty $\Delta \mu_{S}$, is

$$
\begin{aligned}
\mathcal{P}\left(n_{o} ; \mu_{B}, \Delta \mu_{B} ; \mu_{S}, \Delta \mu_{S}\right) & =\frac{1}{R} \int_{0}^{\infty} \int_{0}^{\infty} d \mu_{B}^{\prime} d \mu_{S}^{\prime} \\
& \times \exp \left(-\frac{\left(\mu_{B}-\mu_{B}^{\prime}\right)^{2}}{2\left(\Delta \mu_{B}\right)^{2}}\right) \exp \left(-\frac{\left(\mu_{S}-\mu_{S}^{\prime}\right)^{2}}{2\left(\Delta \mu_{S}\right)^{2}}\right) \\
& \times \frac{\exp \left(-\left(\mu_{B}^{\prime}+\mu_{S}^{\prime}\right)\right) \sum_{n=0}^{n_{o}} \frac{\left(\mu_{B}^{\prime}+\mu_{S}^{\prime}\right)^{n}}{n !}}{\exp \left(-\mu_{B}^{\prime}\right) \sum_{n=0}^{n_{o}} \frac{\left(\mu_{B}^{\prime}\right)^{n}}{n !}},
\end{aligned}
$$

where

$$
R=\int_{0}^{\infty} \int_{0}^{\infty} d \mu_{B}^{\prime} d \mu_{S}^{\prime} \exp \left(-\frac{\left(\mu_{B}-\mu_{B}^{\prime}\right)^{2}}{2\left(\Delta \mu_{B}\right)^{2}}\right) \exp \left(-\frac{\left(\mu_{S}-\mu_{S}^{\prime}\right)^{2}}{2\left(\Delta \mu_{S}\right)^{2}}\right) .
$$

The expected numbers of events, $\mu_{B}$ and $\mu_{S}$, were calculated from the transverse 
mass templates obtained by fitting the Run $1 \mathrm{~B} W / W^{\prime} \rightarrow \mu \nu$ event candidates with the nominal transverse mass lineshapes as described in Section 7.2.4. The systematic uncertainties on $\mu_{B}$ were calculated from the transverse mass templates obtained by fitting the Run 1B $W / W^{\prime} \rightarrow \mu \nu$ event candidates with the transverse mass lineshapes that included systematic effects, similarly to what is described in Section 7.2.4. The uncertainty of each systematic effect, $\delta \mu_{B}$, was calculated as

$$
\delta \mu_{B}=\left(\left|\mu_{B+}-\mu_{B}\right|+\left|\mu_{B-}-\mu_{B}\right|\right) / 2
$$

where $\mu_{B \pm}$ is the number of expected background events from the Monte Carlo sample with $\pm 1 \sigma$ systematic uncertainty variation. Each systematic uncertainty $\delta \mu_{B}$ as well as the statistical uncertainty was summed in quadrature to obtain the total uncertainty $\Delta \mu_{B}$. Similarly, each systematic uncertainty for $\mu_{S}, \delta \mu_{S}$, was calculated for each systematic effect as

$$
\delta \mu_{S}=\left(\left|\mu_{S+}-\mu_{S}\right|+\left|\mu_{S-}-\mu_{S}\right|\right) / 2
$$

where $\mu_{S}$ and $\mu_{S \pm}$ were calculated from the transverse mass templates of the signal from the nominal and the $\pm 1 \sigma$ systematic uncertainty variation of the transverse mass lineshapes, respectively. Again, the total systematic uncertainty for $\mu_{S}, \Delta \mu_{S}$, was calculated by adding $\delta \mu_{S}$ for each systematic effect and the statistic uncertainty in quadrature. Here, it was assumed that $\delta \mu_{B}$ and $\delta \mu_{S}$ were not correlated.

Table A.1 shows the expected number of events and the number of observed events in the transverse mass regions $M_{T}>200,>250,>300,>350$, and $>400 \mathrm{GeV} / c^{2}$. The probability of observing equal to or less than $n_{0}$ events with the number of expected background events $\mu_{B}$ with its uncertainty $\Delta \mu_{B}$ and the number of expected signal events $\mu_{S}$ with its uncertainty $\Delta \mu_{B}$ is $1-\mathcal{P}\left(n_{0} ; \mu_{B}, \Delta \mu_{B} ; \mu_{S}, \Delta \mu_{S}\right)$. Figure A.1 shows the probability of observing equal to or less than $n_{0}$ events as a function of $M_{W^{\prime}}$. The intercept at $\mathcal{P}=5 \%$ gives an exclusion at $95 \% \mathrm{CL}$. The counting cutoff at $M_{T}=300 \mathrm{GeV} / c^{2}$ gives the most stringent mass limit at $M_{W^{\prime}}>650 \mathrm{GeV} / c^{2}$, which is consistent with the results presented in Section B. 


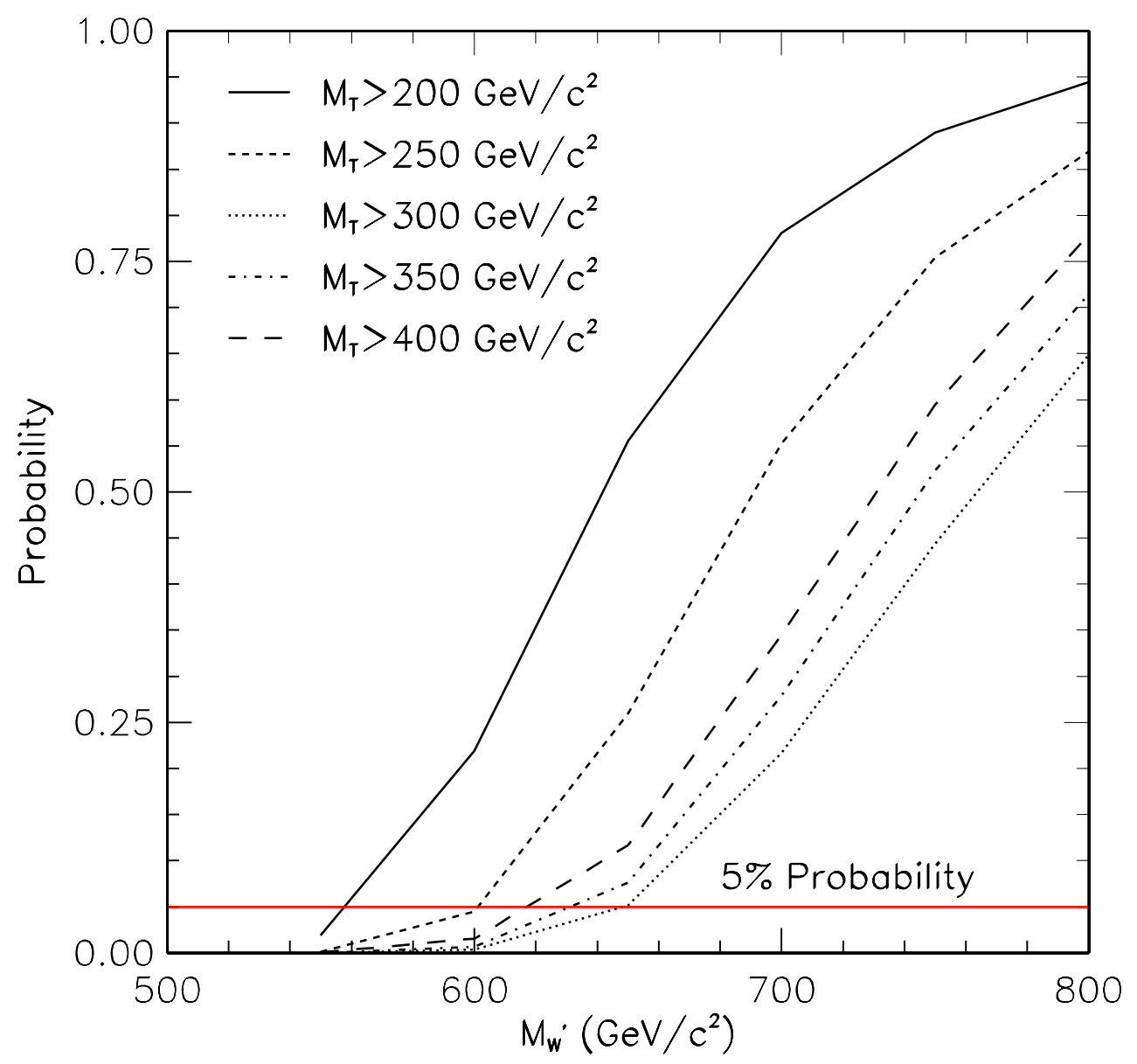

Figure A.1: The $W^{\prime}$ boson mass limits from the counting experiment for different transverse mass cutoffs. The intercepts at the $5 \%$ probability give the $95 \%$ CL limits. The highest mass limit is $M_{W^{\prime}}>650 \mathrm{GeV} / c^{2}$, counting the events in the region $M_{T}>300 \mathrm{GeV} / c^{2}$. 


\begin{tabular}{|c|ccccc|}
\hline$M_{W^{\prime}}\left(\mathrm{GeV} / c^{2}\right)$ & \multicolumn{5}{|c|}{ Number of Events in $M_{T}\left(\mathrm{GeV} / c^{2}\right)$} \\
& $>200$ & $>250$ & $>300$ & $>350$ & $>400$ \\
\hline \hline 550 & $13.9 \pm 1.0$ & $13.5 \pm 1.0$ & $12.7 \pm 0.9$ & $11.5 \pm 0.9$ & $9.64 \pm 0.8$ \\
600 & $7.55 \pm 0.57$ & $7.35 \pm 0.55$ & $7.06 \pm 0.54$ & $6.55 \pm 0.50$ & $5.77 \pm 0.45$ \\
650 & $4.14 \pm 0.34$ & $4.02 \pm 0.33$ & $3.87 \pm 0.32$ & $3.65 \pm 0.31$ & $3.32 \pm 0.29$ \\
700 & $2.27 \pm 0.21$ & $2.20 \pm 0.20$ & $2.12 \pm 0.20$ & $2.01 \pm 0.18$ & $1.86 \pm 0.17$ \\
750 & $1.27 \pm 0.12$ & $1.21 \pm 0.11$ & $1.16 \pm 0.11$ & $1.11 \pm 0.10$ & $1.04 \pm 0.10$ \\
800 & $0.71 \pm 0.07$ & $0.67 \pm 0.07$ & $0.64 \pm 0.06$ & $0.60 \pm 0.06$ & $0.57 \pm 0.05$ \\
\hline \hline Background & $9.63 \pm 0.77$ & $3.83 \pm 0.47$ & $1.81 \pm 0.30$ & $0.96 \pm 0.21$ & $0.56 \pm 0.16$ \\
\hline \hline Observed & 13 & 5 & 1 & 1 & 1 \\
\hline
\end{tabular}

Table A.1: The expected numbers of events for the background $\left(\mu_{B}\right)$ and the signal $\left(\mu_{S}\right)$ for different transverse mass regions (in $\left.\mathrm{GeV} / c^{2}\right)$ in Run 1B. Also shown is the number of observed events $\left(n_{0}\right)$. 


\section{Appendix B}

\section{Individual Limits from Run 1A and Run 1B}

The limits on the $W^{\prime}$ production cross section were calculated for Run $1 \mathrm{~A}$ and Run $1 \mathrm{~B}$ separately as a check of the efficiencies, acceptances, and background calculations. The likelihood distributions were obtained in the same way as described in Section 7.2 but separately for Run 1A and Run 1B using their respectively configured transverse mass lineshapes. The systematic uncertainty smearing of the likelihood distributions for Run $1 \mathrm{~A}$ was performed using the the systematic uncertainties calculated for Run 1B. The $95 \%$ CL limits on $\sigma \cdot \mathcal{B}\left(W^{\prime} \rightarrow \mu \nu\right)$ and $\lambda^{2} \cdot \mathcal{B}\left(W^{\prime} \rightarrow \mu \nu\right)$ are shown in Figures B.1 and B.2, respectively. The lower mass limits are

$$
M_{W^{\prime}}>540 \mathrm{GeV} / c^{2} \text { (Run } 1 \mathrm{~A} \text { at } 95 \% \mathrm{CL} \text { ) }
$$

and

$$
M_{W^{\prime}}>641 \mathrm{GeV} / c^{2}(\text { Run 1B at } 95 \% \mathrm{CL}) \text {, }
$$

assuming the standard model strength couplings and branching fraction. 


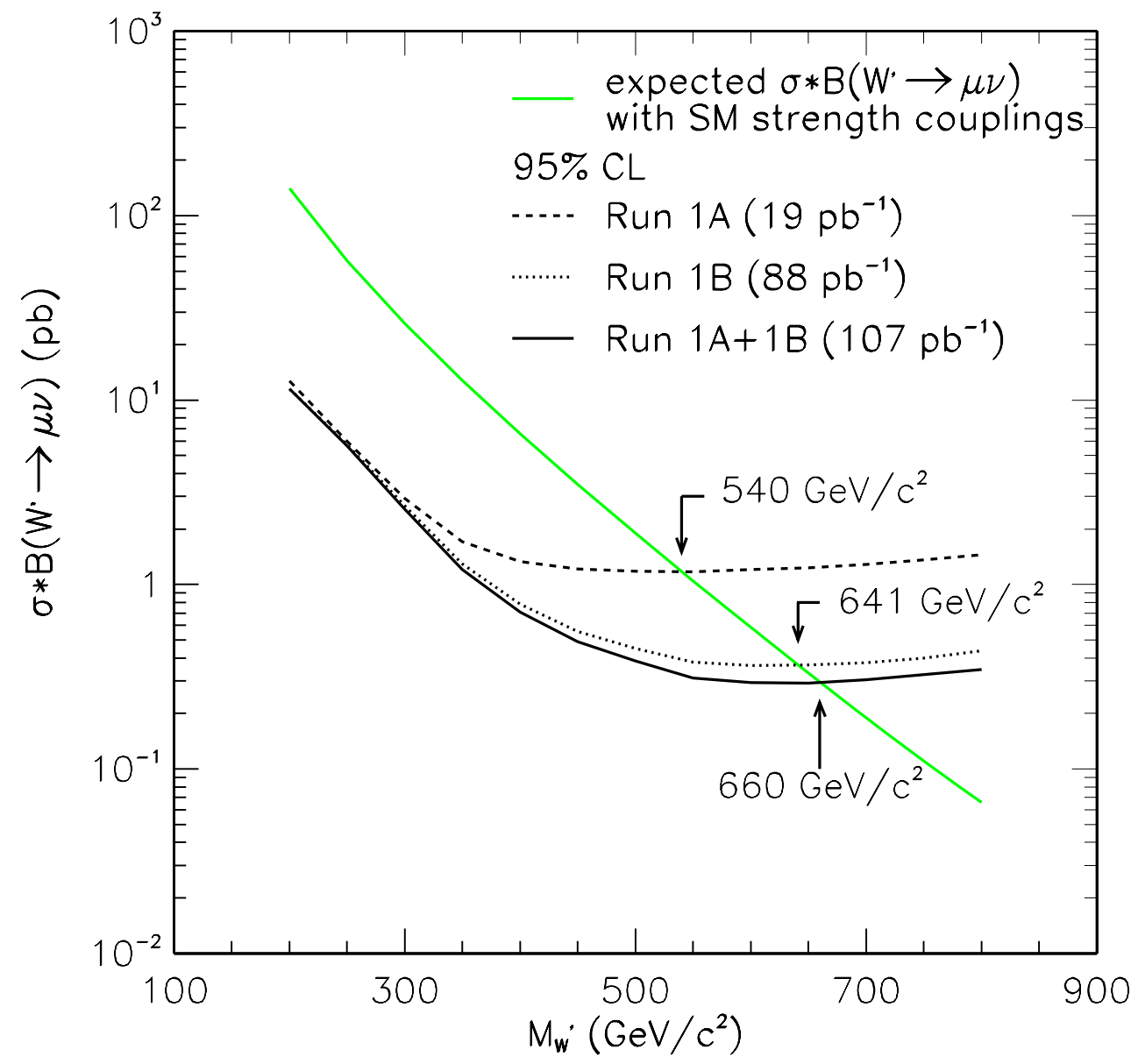

Figure B.1: The $95 \%$ CL limits on $\sigma \cdot \mathcal{B}\left(W^{\prime} \rightarrow \mu \nu\right)$ vs $M_{W^{\prime}}$ for Run $1 \mathrm{~A}$, Run 1B, and Run $1 \mathrm{~A}$ and Run $1 \mathrm{~B}$ combined (Run $1 \mathrm{~A}+1 \mathrm{~B}$ ). The regions above the $95 \% \mathrm{CL}$ curves are excluded. 


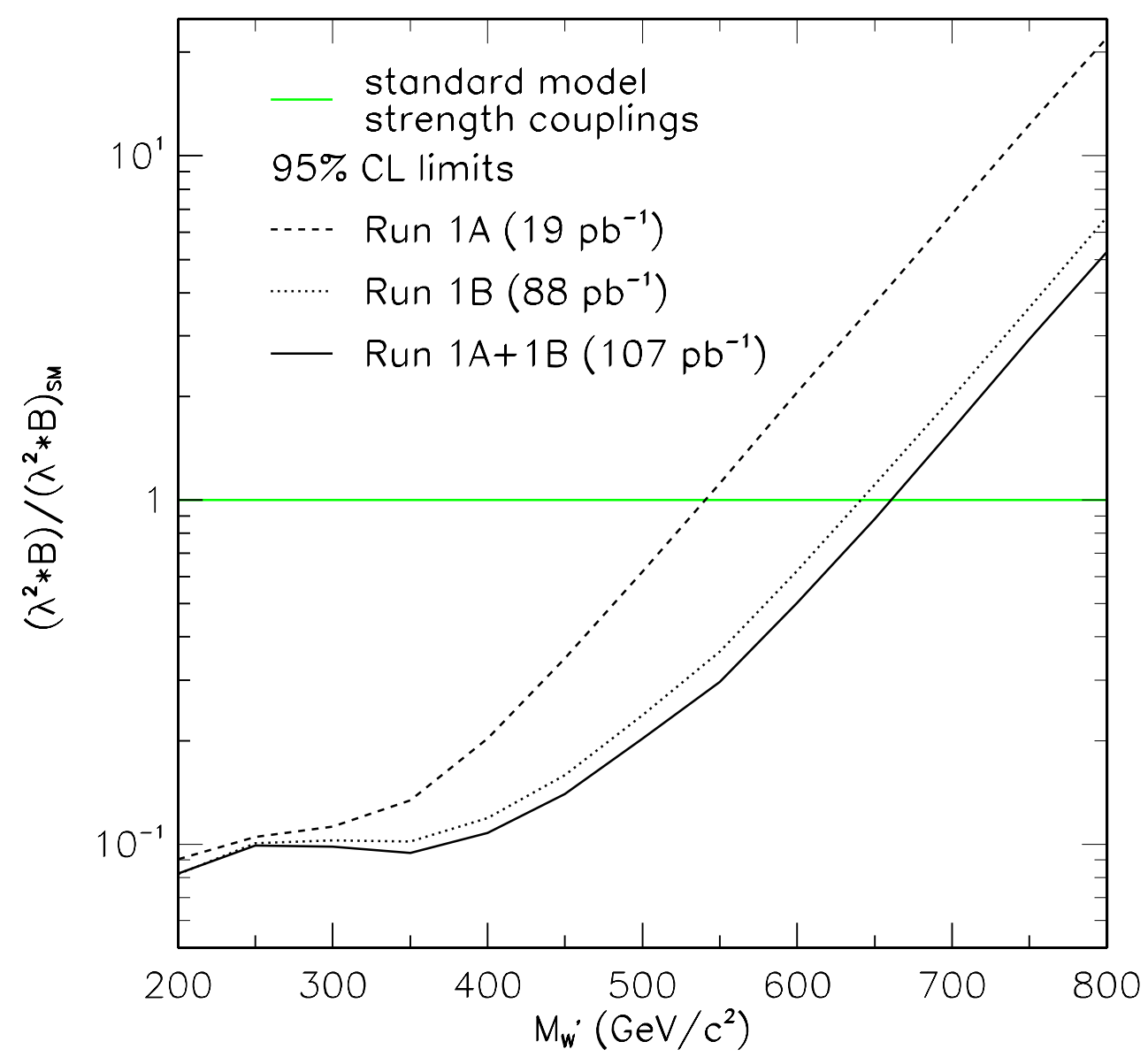

Figure B.2: Couplings strength times branching fraction limits for Run 1A, Run 1B, and Run $1 \mathrm{~A}$ and Run $1 \mathrm{~B}$ combined (Run $1 \mathrm{~A}+1 \mathrm{~B}$ ) as a function of $M_{W^{\prime}}$. The regions above the curves are excluded at $95 \%$ CL. 


\section{Appendix $\mathrm{C}$}

\section{The CDF Collaboration}

F. Abe, ${ }^{17}$ H. Akimoto, ${ }^{39}$ A. Akopian, ${ }^{31}$ M. G. Albrow, ${ }^{7}$ A. Amadon, ${ }^{5}$ S. R. Amendolia, ${ }^{27}$ D. Amidei, ${ }^{20}$ J. Antos,${ }^{33}$ S. Aota,${ }^{37}$ G. Apollinari,,${ }^{31}$ T. Arisawa,${ }^{39}$ T. Asakawa, ${ }^{37}$ W. Ashmanskas,${ }^{5}$ M. Atac,${ }^{7}$ P. Azzi-Bacchetta, ${ }^{25}$ N. Bacchetta, ${ }^{25}$ S. Bagdasarov, ${ }^{31}$ M. W. Bailey, ${ }^{22}$ P. de Barbaro, ${ }^{30}$ A. Barbaro-Galtieri, ${ }^{18}$ V. E. Barnes ${ }^{29}$ B. A. Barnett ${ }^{15}$ M. Barone ${ }^{9}$ G. Bauer, ${ }^{19}$ T. Baumann, ${ }^{11}$ F. Bedeschi, ${ }^{27}$ S. Behrends, ${ }^{3}$ S. Belforte,${ }^{27}$ G. Bellettini, ${ }^{27}$ J. Bellinger, ${ }^{40}$ D. Benjamin, ${ }^{35}$ J. Bensinger, ${ }^{3}$ A. Beretvas,${ }^{7}$ J. P. Berge,${ }^{7}$ J. Berryhill,${ }^{5}$ S. Bertolucci, ${ }^{9}$ S. Bettelli, ${ }^{27}$ B. Bevensee, ${ }^{26}$ A. Bhatti,${ }^{31}$ K. Biery, ${ }^{7}$ C. Bigongiari, ${ }^{27}$ M. Binkley, ${ }^{7}$ D. Bisello, ${ }^{25}$ R. E. Blair, ${ }^{1}$ C. Blocker,${ }^{3}$ K. Bloom,${ }^{20}$ S. Blusk,${ }^{30}$ A. Bodek,${ }^{30}$ W. Bokhari, ${ }^{26}$ G. Bolla,${ }^{29}$ Y. Bonushkin, ${ }^{4}$ D. Bortoletto, ${ }^{29}$ J. Boudreau, ${ }^{28}$ L. Breccia,${ }^{2}$ C. Bromberg, ${ }^{21}$ N. Bruner, ${ }^{22}$ R. Brunetti, ${ }^{2}$ E. Buckley-Geer, ${ }^{7}$ H. S. Budd ${ }^{30}$ K. Burkett, ${ }^{11}$ G. Busetto, ${ }^{25}$ A. Byon-Wagner, ${ }^{7}$ K. L. Byrum, ${ }^{1}$ M. Campbell, ${ }^{20}$ A. Caner ${ }^{27}$ W. Carithers, ${ }^{18}$ D. Carlsmith,${ }^{40}$ J. Cassada, ${ }^{30}$ A. Castro, ${ }^{25}$ D. Cauz,${ }^{36}$ A. Cerri, ${ }^{27}$ P. S. Chang, ${ }^{33}$ P. T. Chang, ${ }^{33}$ H. Y. Chao, ${ }^{33}$ J. Chapman, ${ }^{20}$ M. -T. Cheng, ${ }^{33}$ M. Chertok, ${ }^{34}$ G. Chiarelli, ${ }^{27}$ C. N. Chiou,${ }^{33}$ F. Chlebana,${ }^{7}$ L. Christofek, ${ }^{13}$ R. Cropp,${ }^{14}$ M. L. Chu, ${ }^{33}$ S. Cihangir, ${ }^{7}$ A. G. Clark, ${ }^{10}$ M. Cobal,${ }^{27}$ E. Cocca, ${ }^{27}$ M. Contreras, ${ }^{5}$ J. Conway, ${ }^{32}$ J. Cooper ${ }^{7}$ M. Cordelli, ${ }^{9}$ D. Costanzo, ${ }^{27}$ C. Couyoumtzelis, ${ }^{10}$ D. Cronin-Hennessy ${ }^{6}$ R. Culbertson,${ }^{5}$ D. Dagenhart,${ }^{38}$ T. Daniels,${ }^{19}$ F. DeJongh, ${ }^{7}$ S. Dell'Agnello, ${ }^{9}$ M. Dell'Orso,${ }^{27}$ R. Demina, ${ }^{7}$ L. Demortier, ${ }^{31}$ M. Deninno, ${ }^{2}$ 
P. F. Derwent,${ }^{7}$ T. Devlin, ${ }^{32}$ J. R. Dittmann,${ }^{6}$ S. Donati, ${ }^{27}$ J. Done,${ }^{34}$ T. Dorigo,${ }^{25}$ N. Eddy,${ }^{13}$ K. Einsweiler, ${ }^{18}$ J. E. Elias,${ }^{7}$ R. Ely,${ }^{18}$ E. Engels, Jr. ${ }^{28}$ W. Erdmann, ${ }^{7}$ D. Errede,${ }^{13}$ S. Errede,${ }^{13}$ Q. Fan,${ }^{30}$ R. G. Feild,${ }^{41}$ Z. Feng,,${ }^{15}$ C. Ferretti, ${ }^{27}$ I. Fiori, ${ }^{2}$ B. Flaugher, ${ }^{7}$ G. W. Foster ${ }^{7}$ M. Franklin, ${ }^{11}$ J. Freeman,${ }^{7}$ J. Friedman,${ }^{19}$ H. Frisch,${ }^{5}$ Y. Fukui,${ }^{17}$ S. Gadomski ${ }^{14}$ S. Galeotti,${ }^{27}$ M. Gallinaro, ${ }^{26}$ O. Ganel,${ }^{35}$ M. GarciaSciveres, ${ }^{18}$ A. F. Garfinkel, ${ }^{29}$ C. Gay ${ }^{41}$ S. Geer, ${ }^{7}$ D. W. Gerdes,${ }^{20}$ P. Giannetti, ${ }^{27}$ N. Giokaris,${ }^{31}$ P. Giromini,${ }^{9}$ G. Giusti, ${ }^{27}$ M. Gold ${ }^{22}$ A. Gordon, ${ }^{11}$ A. T. Goshaw,${ }^{6}$ Y. Gotra, ${ }^{28}$ K. Goulianos, ${ }^{31}$ H. Grassmann, ${ }^{36}$ C. Green, ${ }^{29}$ L. Groer,${ }^{32}$ C. GrossoPilcher, ${ }^{5}$ G. Guillian, ${ }^{20}$ J. Guimaraes da Costa, ${ }^{15}$ R. S. Guo,${ }^{33}$ C. Haber, ${ }^{18}$ E. Hafen, ${ }^{19}$ S. R. Hahn,${ }^{7}$ R. Hamilton, ${ }^{11}$ T. Handa,${ }^{12}$ R. Handler ${ }^{40}$ W. Hao,${ }^{35}$ F. Happacher,${ }^{9}$ K. Hara,${ }^{37}$ A. D. Hardman, ${ }^{29}$ R. M. Harris, ${ }^{7}$ F. Hartmann, ${ }^{16}$ J. Hauser, ${ }^{4}$ E. Hayashi, ${ }^{37}$ J. Heinrich, ${ }^{26}$ A. Heiss, ${ }^{16}$ B. Hinrichsen,,${ }^{14}$ K. D. Hoffman, ${ }^{29}$ C. Holck, ${ }^{26}$ R. Hollebeek,${ }^{26}$ L. Holloway, ${ }^{13}$ Z. Huang, ${ }^{20}$ B. T. Huffman, ${ }^{28}$ R. Hughes,${ }^{23}$ J. Huston, ${ }^{21}$ J. Huth, ${ }^{11}$ H. Ikeda, ${ }^{37}$ M. Incagli, ${ }^{27}$ J. Incandela,${ }^{7}$ G. Introzzi, ${ }^{27}$ J. Iwai, ${ }^{39}$ Y. Iwata, ${ }^{12}$ E. James,${ }^{20}$ H. Jensen,${ }^{7}$ U. Joshi, ${ }^{7}$ E. Kajfasz,${ }^{25}$ H. Kambara, ${ }^{10}$ T. Kamon, ${ }^{34}$ T. Kaneko, ${ }^{37}$ K. Karr, ${ }^{38}$ H. Kasha,${ }^{41}$ Y. Kato, ${ }^{24}$ T. A. Keaffaber, ${ }^{29}$ K. Kelley, ${ }^{19}$ R. D. Kennedy, ${ }^{7}$ R. Kephart,${ }^{7}$ D. Kestenbaum, ${ }^{11}$ D. Khazins, ${ }^{6}$ T. Kikuchi, ${ }^{37}$ B. J. Kim, ${ }^{27}$ H. S. Kim, ${ }^{14}$ S. H. Kim,${ }^{37}$ Y. K. Kim, ${ }^{18}$ L. Kirsch, ${ }^{3}$ S. Klimenko, ${ }^{8}$ D. Knoblauch, ${ }^{16}$ P. Koehn, ${ }^{23}$ A. Köngeter, ${ }^{16}$ K. Kondo, ${ }^{37}$ J. Konigsberg, ${ }^{8}$ K. Kordas, ${ }^{14}$ A. Korytov,${ }^{8}$ E. Kovacs,${ }^{1}$ W. Kowald,${ }^{6}$ J. Kroll, ${ }^{26}$ M. Kruse,${ }^{30}$ S. E. Kuhlmann, ${ }^{1}$ E. Kuns, ${ }^{32}$ K. Kurino, ${ }^{12}$ T. Kuwabara,${ }^{37}$ A. T. Laasanen,${ }^{29}$ S. Lami,${ }^{27}$ S. Lammel,${ }^{7}$ J. I. Lamoureux,${ }^{3}$ M. Lancaster, ${ }^{18}$ M. Lanzoni, ${ }^{27}$ G. Latino, ${ }^{27}$ T. LeCompte, ${ }^{1}$ S. Leone,${ }^{27}$ J. D. Lewis,${ }^{7}$ M. Lingren,${ }^{4}$ T. M. Liss,${ }^{13}$ J. B. Liu,${ }^{30}$ Y. C. Liu,${ }^{33}$ N. Lockyer, ${ }^{26}$ O. Long, ${ }^{26}$ M. Loreti, ${ }^{25}$ D. Lucchesi, ${ }^{27}$ P. Lukens, ${ }^{7}$ S. Lusin,${ }^{40}$ J. Lys,${ }^{18}$ K. Maeshima,${ }^{7}$ P. Maksimovic,${ }^{11}$ M. Mangano, ${ }^{27}$ M. Mariotti ${ }^{25}$ J. P. Marriner,${ }^{7}$ G. Martignon, ${ }^{25}$ A. Martin, ${ }^{41}$ J. A. J. Matthews ${ }^{22}$ P. Mazzanti, ${ }^{2}$ K. McFarland, ${ }^{30}$ P. McIntyre, ${ }^{34}$ P. Melese ${ }^{31}$ M. Menguzzato,${ }^{25}$ A. Menzione,${ }^{27}$ E. Meschi, ${ }^{27}$ S. Metzler ${ }^{26}$ C. Miao, ${ }^{20}$ T. Miao,${ }^{7}$ G. Michail, ${ }^{11}$ R. Miller,${ }^{21}$ H. Minato,${ }^{37}$ S. Miscetti ${ }^{9}$ M. Mishina,${ }^{17}$ S. Miyashita,${ }^{37}$ N. Moggi, ${ }^{27}$ E. Moore,${ }^{22}$ Y. Morita,${ }^{17}$ A. Mukherjee, ${ }^{7}$ T. Muller, ${ }^{16}$ A. Munar ${ }^{27}$ P. Murat,${ }^{27}$ S. Murgia, ${ }^{21}$ M. Musy,${ }^{36}$ H. Nakada,${ }^{37}$ T. Nakaya,${ }^{5}$ I. Nakano, ${ }^{12}$ C. Nelson,${ }^{7}$ D. Neuberger,${ }^{16}$ C. Newman- 
Holmes, ${ }^{7}$ C.-Y. P. Ngan, ${ }^{19}$ L. Nodulman, ${ }^{1}$ A. Nomerotski, ${ }^{8}$ S. H. Oh, ${ }^{6}$ T. Ohmoto, ${ }^{12}$ T. Ohsugi, ${ }^{12}$ R. Oishi,${ }^{37}$ M. Okabe,${ }^{37}$ T. Okusawa, ${ }^{24}$ J. Olsen, ${ }^{40}$ C. Pagliarone,${ }^{27}$ R. Paoletti, ${ }^{27}$ V. Papadimitriou, ${ }^{35}$ S. P. Pappas, ${ }^{41}$ N. Parashar, ${ }^{27}$ A. Parri,${ }^{9}$ J. Patrick,${ }^{7}$ G. Pauletta, ${ }^{36}$ M. Paulini, ${ }^{18}$ A. Perazzo,${ }^{27}$ L. Pescara, ${ }^{25}$ M. D. Peters,${ }^{18}$ T. J. Phillips, ${ }^{6}$ G. Piacentino, ${ }^{27}$ M. Pillai, ${ }^{30}$ K. T. Pitts,${ }^{7}$ R. Plunkett,${ }^{7}$ A. Pompos,${ }^{29}$ L. Pondrom,${ }^{40}$ J. Proudfoot, ${ }^{1}$ F. Ptohos, ${ }^{11}$ G. Punzi, ${ }^{27}$ K. Ragan, ${ }^{14}$ D. Reher, ${ }^{18}$ M. Reischl, ${ }^{16}$ A. Ribon, ${ }^{25}$ F. Rimondi, ${ }^{2}$ L. Ristori, ${ }^{27}$ W. J. Robertson, ${ }^{6}$ A. Robinson, ${ }^{14}$ T. Rodrigo, ${ }^{27}$ S. Rolli, ${ }^{38}$ L. Rosenson, ${ }^{19}$ R. Roser, ${ }^{13}$ T. Saab, ${ }^{14}$ W. K. Sakumoto, ${ }^{30}$ D. Saltzberg, ${ }^{4}$ A. Sansoni, ${ }^{9}$ L. Santi, ${ }^{66}$ H. Sato, ${ }^{37}$ P. Schlabach,${ }^{7}$ E. E. Schmidt, ${ }^{7}$ M. P. Schmidt,${ }^{41}$ A. Scott, ${ }^{4}$ A. Scribano, ${ }^{27}$ S. Segler,${ }^{7}$ S. Seidel, ${ }^{22}$ Y. Seiya, ${ }^{37}$ F. Semeria, ${ }^{2}$ T. Shah, ${ }^{19}$ M. D. Shapiro, ${ }^{18}$ N. M. Shaw, ${ }^{29}$ P. F. Shepard, ${ }^{28}$ T. Shibayama, ${ }^{37}$ M. Shimojima, ${ }^{37}$ M. Shochet, ${ }^{5}$ J. Siegrist, ${ }^{18}$ A. Sill, ${ }^{35}$ P. Sinervo, ${ }^{14}$ P. Singh, ${ }^{13}$ K. Sliwa, ${ }^{38}$ C. Smith, ${ }^{15}$ F. D. Snider, ${ }^{15}$ J. Spalding,${ }^{7}$ T. Speer,${ }^{10}$ P. Sphicas, ${ }^{19}$ F. Spinella, ${ }^{27}$ M. Spiropulu, ${ }^{11}$ L. Spiegel, ${ }^{7}$ L. Stanco, ${ }^{25}$ J. Steele, ${ }^{40}$ A. Stefanini, ${ }^{27}$ R. Ströhmer, ${ }^{7 a}$ J. Strologas, ${ }^{13}$ F. Strumia, ${ }^{10}$ D. Stuart,${ }^{7}$ K. Sumorok, ${ }^{19}$ J. Suzuki, ${ }^{37}$ T. Suzuki, ${ }^{37}$ T. Takahashi, ${ }^{24}$ T. Takano, ${ }^{24}$ R. Takashima, ${ }^{12}$ K. Takikawa, ${ }^{37}$ M. Tanaka, ${ }^{37}$ B. Tannenbaum, ${ }^{4}$ F. Tartarelli, ${ }^{27}$ W. Taylor, ${ }^{14}$ M. Tecchio, ${ }^{20}$ P. K. Teng, ${ }^{33}$ Y. Teramoto, ${ }^{24}$ K. Terashi, ${ }^{37}$ S. Tether, ${ }^{19}$ D. Theriot, ${ }^{7}$ T. L. Thomas, ${ }^{22}$ R. Thurman-Keup, ${ }^{1}$ M. Timko, ${ }^{38}$ P. Tipton, ${ }^{30}$ A. Titov, ${ }^{31}$ S. Tkaczyk, ${ }^{7}$ D. Toback, ${ }^{5}$ K. Tollefson, ${ }^{30}$ A. Tollestrup,${ }^{7}$ H. Toyoda, ${ }^{24}$ W. Trischuk, ${ }^{14}$ J. F. de Troconiz, ${ }^{11}$ S. Truitt,${ }^{20}$ J. Tseng, ${ }^{19}$ N. Turini, ${ }^{27}$ T. Uchida, ${ }^{37}$ F. Ukegawa, ${ }^{26}$ J. Valls, ${ }^{32}$ S. C. van den Brink, ${ }^{15}$ S. Vejcik, III, ${ }^{20}$ G. Velev ${ }^{27}$ I. Volobouev, ${ }^{18}$ R. Vidal, ${ }^{7}$ R. Vilar, ${ }^{7 a}$ D. Vucinic,${ }^{19}$ R. G. Wagner,${ }^{1}$ R. L. Wagner, ${ }^{7}$ J. Wahl,${ }^{5}$ N. B. Wallace, ${ }^{27}$ A. M. Walsh, ${ }^{32}$ C. Wang, ${ }^{6}$ C. H. Wang, ${ }^{33}$ M. J. Wang, ${ }^{33}$ A. Warburton, ${ }^{14}$ T. Watanabe, ${ }^{37}$ T. Watts, ${ }^{32}$ R. Webb, ${ }^{34}$ C. Wei,${ }^{6}$ H. Wenzel, ${ }^{16}$ W. C. Wester, III, ${ }^{7}$ A. B. Wicklund,${ }^{1}$ E. Wicklund,${ }^{7}$ R. Wilkinson, ${ }^{26}$ H. H. Williams, ${ }^{26}$ P. Wilson, ${ }^{7}$ B. L. Winer,${ }^{23}$ D. Winn, ${ }^{20}$ D. Wolinski, ${ }^{20}$ J. Wolinski, ${ }^{21}$ S. Worm,${ }^{22}$ X. Wu,${ }^{10}$ J. Wyss,${ }^{27}$ A. Yagil,${ }^{7}$ W. Yao, ${ }^{18}$ K. Yasuoka, ${ }^{37}$ G. P. Yeh, ${ }^{7}$ P. Yeh, ${ }^{33}$ J. Yoh,${ }^{7}$ C. Yosef,${ }^{21}$ T. Yoshida, ${ }^{24}$ I. Yu, ${ }^{7}$ A. Zanetti, ${ }^{36}$ F. Zetti, ${ }^{27}$ and S. Zucchelli ${ }^{2}$

(CDF Collaboration) 
1 Argonne National Laboratory, Argonne, Illinois 60439

2 Istituto Nazionale di Fisica Nucleare, University of Bologna, I-40127 Bologna, Italy

3 Brandeis University, Waltham, Massachusetts 02254

4 University of California at Los Angeles, Los Angeles, California 90024

5 University of Chicago, Chicago, Illinois 60637

6 Duke University, Durham, North Carolina 27708

7 Fermi National Accelerator Laboratory, Batavia, Illinois 60510

8 University of Florida, Gainesville, Florida 32611

9 Laboratori Nazionali di Frascati, Istituto Nazionale di Fisica Nucleare, I-00044 Frascati, Italy

10 University of Geneva, CH-1211 Geneva 4, Switzerland

11 Harvard University, Cambridge, Massachusetts 02138

12 Hiroshima University, Higashi-Hiroshima 724, Japan

13 University of Illinois, Urbana, Illinois 61801

14 Institute of Particle Physics, McGill University, Montreal H3A 2T8, and University of Toronto, Toronto M5S 1A7, Canada

15 The Johns Hopkins University, Baltimore, Maryland 21218

16 Institut für Experimentelle Kernphysik, Universität Karlsruhe, 76128 Karlsruhe, Germany 17 National Laboratory for High Energy Physics (KEK), Tsukuba, Ibaraki 305, Japan

18

Ernest Orlando Lawrence Berkeley National Laboratory, Berkeley, California 94720

19 Massachusetts Institute of Technology, Cambridge, Massachusetts 02139

20 University of Michigan, Ann Arbor, Michigan 48109

21 Michigan State University, East Lansing, Michigan 48824

22 University of New Mexico, Albuquerque, New Mexico 87131

23 The Ohio State University, Columbus, Ohio 43210

24 Osaka City University, Osaka 588, Japan

25 Universita di Padova, Istituto Nazionale di Fisica Nucleare, Sezione di Padova, I-35131 Padova, Italy

26 University of Pennsylvania, Philadelphia, Pennsylvania 19104

Istituto Nazionale di Fisica Nucleare, University and Scuola Normale Superiore of Pisa, I-56100 Pisa, Italy

28 University of Pittsburgh, Pittsburgh, Pennsylvania 15260

29 Purdue University, West Lafayette, Indiana 47907

30 University of Rochester, Rochester, New York 14627 
Rockefeller University, New York, New York 10021

32

Rutgers University, Piscataway, New Jersey 08855

33 Academia Sinica, Taipei, Taiwan 11530, Republic of China

34 Texas A 1 M University, College Station, Texas 77843

35 Texas Tech University, Lubbock, Texas 79409

36 Istituto Nazionale di Fisica Nucleare, University of Trieste/ Udine, Italy

37 University of Tsukuba, Tsukuba, Ibaraki 305, Japan

38 Tufts University, Medford, Massachusetts 02155

39 Waseda University, Tokyo 169, Japan

40 University of Wisconsin, Madison, Wisconsin 53706

41 Yale University, New Haven, Connecticut 06520 


\section{Bibliography}

[1] W. Pauli, Handbuch der Physik 24, 1 (1933).

[2] E. Fermi, Nuovo Cimento 11, 1 (1934); E. Fermi, Z. Phys. 88, 161 (1934).

[3] T. D. Lee and C. N. Yang, Phys. Rev. 104, 254 (1956).

[4] C. S. Wu et al., Phys. Rev. 105, 1413 (1957).

[5] M. Goldhaber, L. Grodzins, and A. W. Sunyar, Phys. Rev. 109, 1015 (1958).

[6] R. P. Feynman and M. Gell-Mann, Phys. Rev. 109, 193 (1958); E. G. C. Sudarshan and R. E. Marshak, ibid. 109, 1860 (1958); J. J. Sakurai, Nuovo Cimento 14, 951 (1958).

[7] J. Schwinger, Ann. Phys. 2, 407 (1957).

[8] S. L. Galshow, Nucl. Phys. 22, 579 (1961).

[9] A. Salam and J. C. Ward, Phys. Lett. 13, 168 (1964).

[10] S. Weinberg, Phys. Rev. Lett. 19, 1264 (1967); A. Salam, "Elementary Particle Physics," ed. by N. Svartholm, (Almqvist and Wilsell, Stockholm, 1968).

[11] UA1 Collaboration, G. Arnison et al., Phys. Lett. 109 B, 103 (1983); UA1 Collaboration, G. Arnison et al., ibid. 126 B, 397 (1983).

[12] UA2 Collaboration, M. Banner et al., Phys. Lett. 122 B, 103 (1983).

[13] UA2 Collaboration, P. Bagnaia et al., Phys. Lett. 129 B, 130 (1983). 
[14] J. C. Pati and A. Salam, Phys. Rev. D 10, 275 (1974); R. N. Mohapatra and J. C. Pati, ibid. D 11, 2558 (1975); G. Senjanovic and R. N. Mohapatra, ibid. D 12, $1502(1975)$.

[15] For a detailed discussion on Majorana masses for neutrinos, see R. N. Mohapatra, "Unification and Supersymmetry," (New York, Springer-Verlag, 1992), pp. 145154.

[16] CTEQ Collaboration, H. L. Lai et al., Phys. Rev. D 55, 1280 (1997).

[17] CDF Collaboration, F. Abe et al., Phys. Rev. Lett. 74, 2626 (1995).

[18] G. Altarelli, B. Mele, and M. Ruiz-Altaba, Z. Phys. C 45, 109 (1989).

[19] DØ Collaboration, S. Abachi et al., Phys. Rev. Lett. 76, 3271 (1996).

[20] CDF Collaboration, F. Abe et al., Phys. Rev. Lett. 67, 2609 (1991).

[21] The mass limit goes down to $M_{W_{R}}>251 \mathrm{GeV} / c^{2}$ if the top decay channel is allowed. UA2 Collaboration, J. Alitti et al., Nucl. Phys. B400, 3 (1993).

[22] CDF Collaboration, F. Abe et al., Phys. Rev. D 55, 5263 (1997).

[23] If the $W_{L}-W_{R}$ mixing is not allowed, the mass limit goes up to $M_{W_{R}}>$ $482 \mathrm{GeV} / \mathrm{c}^{2}$. A. Jodidio et al., Phys. Rev. D 34, 1967 (1986); D 37, 237(E) (1988).

[24] J. Imazato et al., Phys. Rev. Lett. 69, 877 (1992).

[25] P. Langacker and S. U. Sankar, Phys. Rev. D 40, 1569 (1989).

[26] R. Barbieri and R. N. Mohapatra, Phys. Rev. D 39, 1229 (1989).

[27] Super-Kamiokande Collaboration, Y. Fukuda et al., Phys. Rev. Lett. 81, 1562 (1998).

[28] CDF Collaboration, F. Abe et al., Phys. Rev. D 50, 5550 (1994).

[29] D. Amidei et al., Nucl. Instrum. Methods Phys. Res. A 350, 73 (1994). 
[30] P. Azzi et al., Nucl. Instrum. Methods Phys. Res. A 360, 137 (1995).

[31] F. Abe et al., Nucl. Instrum. Methods Phys. Res. A 268, 75 (1988).

[32] F. Bedeschi et al., Nucl. Instrum. Methods Phys. Res. A 268, 50 (1988).

[33] L. Balka et al., Nucl. Instrum. Methods Phys. Res. A 267, 272 (1988).

[34] S. Bertolucci et al., Nucl. Instrum. Methods Phys. Res. A 267, 301 (1988).

[35] Y. Fukui et al., Nucl. Instrum. Methods Phys. Res. A 267, 280 (1988).

[36] G. Brandenburg et al., Nucl. Instrum. Methods Phys. Res. A 267, 257 (1988).

[37] S. Cihangir et al., Nucl. Instrum. Methods Phys. Res. A 267, 249 (1988).

[38] J. D. Lewis et al., "The 1992 CDF Muon System Upgrade," CDF Internal Note CDF/PUB/MUON/PUBLIC/2858 (1995), unpublished.

[39] D. Glenzinski and P. Schlabach, "Combined Level 1 and Level 2 High $p_{T}$ Muon Trigger Efficiencies in Run 1B," CDF Internal Note CDF/ANAL/MUON/CDFR/2954 (1995), unpublished; R. Roser and T. M. Liss, "A Measurement of the Trigger Efficiencies for $t \bar{t}$ Events in Run 1B," CDF Internal Note CDF/ANAL/TOP/GROUP/3442 (1996), unpublished.

[40] L. Qi and K. T. Pitts, "The CFT Efficiency for High- $p_{T}$ Tracks in Run 1B," CDF Internal Note CDF/ANAL/TRIGGER/CDFR/4000 (1996), unpublished.

[41] W. F. Badgett, Jr., "Measurement of $\sigma \cdot B(W \rightarrow \mu \nu), \sigma \cdot B\left(Z^{0} \rightarrow \mu^{+} \mu^{-}\right)$and $R_{\mu}=\sigma \cdot B(W \rightarrow \mu \nu) / \sigma \cdot B\left(Z^{0} \rightarrow \mu^{+} \mu^{-}\right)$and Extraction of $B R(W \rightarrow \mu \nu)$ and $\Gamma(W)$ in $p \bar{p}$ Collisions at $\sqrt{s}=1.8 \mathrm{TeV}$," Ph.D. Thesis, University of Michigan (1994).

[42] T. LeCompte, T. M. Liss, and A. Martin, "High $P_{t}$ CMU/CMP Muon Trigger Efficiencies for Run 1A," CDF Internal Note CDF/DOC/MUON/CDFR/2367 (1993), unpublished.

[43] M. K. Pillai, "A Search for New Gauge Bosons in $\bar{p} p$ Collisions at $\sqrt{s}=1.8 \mathrm{TeV}$," Ph.D. Thesis, University of Rochester (1996). 
[44] R. Brun and F. Carninati, "GEANT Detector Description and Simulation Tool," CERN Programming Library Long Writeup W5013 (1993).

[45] T. Sjöstrand, Comput. Phys. Commun. 82, 74 (1994).

[46] P. B. Arnold and R. P. Kauffman, Nucl. Phys. B349, 381 (1991).

[47] Particle Data Group, R. M. Barnett et al., "Review of Particle Physics," Phys. Rev. D 54, 208 (1996).

[48] CDF Collaboration, F. Abe et al., Phys. Rev. Lett. 75, 11 (1995).

[49] CDF Collaboration, F. Abe et al., Phys. Rev. Lett. 66, 2951 (1991); CDF Collaboration, F. Abe et al., ibid. 67, 2937 (1991).

[50] M. H. Reno, Phys. Rev. D 49, 4326 (1994).

[51] M. Lancaster, "Lepton Resolutions for the Run 1B W Mass Analysis," CDF Internal Note CDF/ANAL/ELECTROWEAK/CDFR/4046 (1997), unpublished.

[52] F. E. James and M. Roos, Comput. Phys. Commun. 10, 343 (1975).

[53] Particle Data Group, R. M. Barnett et al., "Review of Particle Physics," Phys. Rev. D 54, 214 (1996).

[54] CDF Collaboration, F. Abe et al., Phys. Rev. Lett. 73, 220 (1994).

[55] Particle Data Group, R. M. Barnett et al., "Review of Particle Physics," Phys. Rev. D 54, 256 (1996).

[56] A. D. Martin, R. G. Roberts, and W. J. Stirling, Phys. Rev. Lett. B 387, 419 (1996).

[57] A. Castro, "Measurement of $t \bar{t}$ Production Cross Section at the Tevatron," FERMILAB-Conf-97/137-E, (1997).

[58] F. Solmitz, Ann. Rev. Nucl. Sci. 14, 375 (1964).

[59] Particle Data Group, R. M. Barnett et al., "Review of Particle Physics," Phys. Rev. D 54, 164 (1996). 
[60] CTEQ Collaboration, J. Botts et al., Phys. Lett. 304 B, 159 (1993).

[61] A. D. Martin, R. G. Roberts, and W. J. Stirling, Phys. Rev. Lett. B 306, 147 (1993); B 309, 492(E) (1993).

[62] J. A. Macdonald and J-M. Poutissou, Physics in Canada Vol. 50, No. 2166 (1994). 